\title{
Analysis and Practice of Uniquely Decodable One-to-One Code
}

\author{
Chin-Fu Liu, Hsiao-feng (Francis) Lu, and Po-Ning Chen
}

\begin{abstract}
In this paper, we consider the so-called uniquely decodable one-to-one code (UDOOC) that is formed by inserting a "comma" indicator, termed the unique word (UW), between consecutive one-to-one codewords for separation. Along this research direction, we first investigate several general combinatorial properties of UDOOCs, in particular the enumeration of the number of UDOOC codewords for any (finite) codeword length. Based on the obtained formula on the number of length- $n$ codewords for a given $U W$, the per-letter average codeword length of UDOOC for the optimal compression of a given source statistics can be computed. Several upper bounds on the average codeword length of such UDOOCs are next established. The analysis on the bounds of average codeword length then leads to two asymptotic bounds for sources having infinitely many alphabets, one of which is achievable and hence tight for a certain source statistics and UW, and the other of which proves the achievability of source entropy rate of UDOOCs when both the block size of source letters for UDOOC compression and UW length go to infinity. Efficient encoding and decoding algorithms for UDOOCs are also given in this paper. Numerical results show that when grouping three English letters as a block, the UDOOCs with $\mathbf{U W}=0001$, 0000, 000001 and 000000 can respectively reach the compression rates of 3.531, 4.089, 4.115, 4.709 bits per English letter (with the lengths of UWs included), where the source stream to be compressed is the book titled Alice's Adventures in Wonderland. In comparison with the first-order Huffman code, the second-order Huffman code, the third-order Huffman code and the Lempel-Ziv code, which respectively achieve the compression rates of 3.940 , $3.585,3.226$ and 6.028 bits per single English letter, the proposed UDOOCs can potentially result in comparable compression rate to the Huffman code under similar decoding complexity and yield a smaller average codeword length than that of the Lempel-Ziv code, thereby confirming the practicability of UDOOCs.
\end{abstract}

\section{INTRODUCTION}

The investigation of lossless source coding can be roughly classified into two categories, one for the compression of a sequence of source letters and the other for a single "one shot" source symbol [7]. A well-known representative for the former is the Huffman code, while the latter is usually referred to as the one-to-one code (OOC).

The Huffman code is an optimal entropy code that can achieve the minimum average codeword length for a given statistics of source letters. It obeys the rule of unique decodability and hence the concatenation of Huffman codewords can be uniquely recovered by the decoder. Although optimal in principle, it may encounter several obstacles in implementation. For example, the rare codewords are exceedingly long

The authors are with the Department of Electrical and Computer Engineering, National Chiao-Tung University (NCTU), Hsinchu 30010, Taiwan (e-mails: hubert.liu.1031@gmail.com, francis@mail.nctu.edu.tw, poning@faculty.nctu.edu.tw). They are also with the Center of Information and Communications Technology of NCTU, Taiwan. in length, thereby hampering the efficiency of decoding. Other practical obstacles include

i) the codebook needs to be pre-stored for encoding and decoding, which might demand a large memory space for sources with moderately large alphabet size,

ii) the decoding of a sequence of codewords must be done in sequential, not in parallel, and

iii) erroneous decoding of one codeword could affect the decoding of subsequent codewords, i.e., error propagation.

In contrast to unique decodability, the OOC only requires an assignment of distinct codewords to the source symbols. It has been studied since 1970s [33] and is shown to achieve an average codeword length smaller than the source entropy minus a nontrivial amount of quantity called anti-redundancy [29]. Various research works over the years have shown that the anti-redundancy can be as large as the logarithm of the source entropy [1], [5], [6], [13], [21], [24], [26], [27], [29][31] In comparison with an entropy coding like Huffman code, the codewords of an OOC can be sequenced alphabetically and hence the practice of an OOC is generally considered to be more computationally convenient.

A question that may arise from the above discussion is whether we could add a "comma" indicator, termed Unique Word (UW) in this paper, in-between consecutive OOC codewords, and use the OOC for the lossless compression of a sequence of source letters. A direct merit of such a structure is that the alphabetically sequenced OOC codewords can be manipulated without a priori stored codebook at both the encoding and decoding ends. This is however achieved at a price of an additional constraint that the "comma" indicator must not appear as an internal subword" in the concatenation of either an OOC codeword with a comma indicator, or a comma indicator with an OOC codeword.

On the one hand, this additional constraint facilitates the fast identification of OOC codewords in a coded bit-stream and makes feasible the subsequent parallel decoding of them. On the other hand, the achievable average codeword length of a UW-forbidden OOC may increase significantly for a bad choice of UWs. Therefore, it is of theoretical importance to investigate the minimum average codeword length of a UWforbidden OOC, in particular the selection of a proper UW that could minimize this quantity. Since the resultant UWforbidden OOC coding system satisfies unique decodability

\footnotetext{
${ }^{1}$ We say $\boldsymbol{a}=a_{1} \ldots a_{m}$ is not an internal subword of $\boldsymbol{b}=b_{1} \ldots b_{n}$ if there does not exist $i$ such that $b_{i} \ldots b_{i+m-1}=\boldsymbol{a}$ for all $1<i<n-m+1$. When the same condition holds for all $1 \leq i \leq n-m+1$ (i.e., with two equalities), we say $\boldsymbol{a}$ is not a subword of $\bar{b}$.
} 
(UD), we will refer to it conveniently as the UDOOC in the sequel.

We would like to point out that the conception of inserting UWs between consecutive words might not be new in existing applications. For example, in the IEEE 802.11 standard for wireless local area networks [3], an entity similar to the UW in a bit-stream has been specified as a boundary indicator for a frame, or as a synchronization support, or as a part of error control mechanism. In written English, punctuation marks and spacing are essential to disambiguate the meaning of sentences. However, a complete theoretical study of the UDOOC conception remains undone. This is therefore the main target of this paper. We now give a formal definition of binary UDOOCs.

Definition 1: Given UW $\boldsymbol{k}=k_{1} k_{2} \ldots k_{L} \in \mathbb{F} \times \cdots \times \mathbb{F}=$ $\mathbb{F}^{L}$, where $\mathbb{F}=\{0,1\}$, we say $\mathcal{C}_{\boldsymbol{k}}(n)$ is a UDOOC of length $n \geq 1$ associated with $\boldsymbol{k}$ if it contains all binary length- $n$ tuples $\boldsymbol{b}=b_{1} \ldots b_{n}$ such that $\boldsymbol{k}$ is not an internal subword of the concatenated bit-stream $\boldsymbol{k} \boldsymbol{b} \boldsymbol{k}$. As a special case, we set $\mathcal{C}_{\boldsymbol{k}}(0):=\{$ null $\} .{ }^{2}$ The overall UDOOC associated with $\boldsymbol{k}$, denoted by $\mathcal{C}_{k}$, is given by

$$
\mathcal{C}_{\boldsymbol{k}}:=\bigcup_{n \geq 0} \mathcal{C}_{\boldsymbol{k}}(n)
$$

For a better comprehension of Definition 1, we next give an example to illustrate how a UDOOC is generated and how it is used in encoding and decoding. The way to count the number of length- $n$ UDOOC codewords will follow.

Example 1: Suppose the UW $\boldsymbol{k}=00$ is chosen. In order to prohibit the concatenation of any UDOOC codeword and UW, regardless of the ordering, from containing 00 as an internal subword, the following constraints must be satisfied.

- Type-I constraints: The UW cannot be a subword of any UDOOC codeword. This means that within any codeword of length $n \geq 1$ :

(C1) " 0 " can only be followed by " 1 ".

(C2) "1" can be followed by either " 0 " or " 1 ".

- Type-II constraints: Besides the type-I constraints, the UW cannot appear as an internal subword, containing the boundary of any UDOOC codeword and UW, regardless of the ordering. This implies that except for the "null" codeword:

(C3) The first bit of a codeword cannot be "0".

(C4) The last bit of a codeword cannot be "0".

By Constraints $(\mathrm{C} 1)-(\mathrm{C} 4)$, we can place the UDOOC codewords on a code tree as shown in Fig. 1, in which each path starting from the root node and ending at a gray-shaded node corresponds to a codeword. Thus, the codewords for UW $=00$ include null, 1, 11, 101, 111, 1011, 1101, 1111, etc. It should be noted that we only show the codewords of length up to four, while the code tree actually can grow indefinitely in depth.

At the decoding stage, suppose the received bit-stream is 00100110010100111100, where we add UWs at both the

\footnotetext{
${ }^{2}$ In our binary UDOOC, it is allowed to place two UWs side-by-side with nothing in-between in order to produce a null codeword.
}



Fig. 1. UDOOC code tree for $\mathrm{UW}=00$.

left and the right ends to indicate the margins of the bitstream. This may facilitate, for example, noncoherent bitstream transmission. Then, the decoder first locates UWs and parses the bit-stream into separate codewords as 1, 11, 101 and 1111, after which the four codewords can be decoded separately (possibly in parallel) to their respective source symbols.

With the code tree representation, the number of length$n$ codewords in a UDOOC code tree can be straightforwardly calculated. Let the "null"-node be placed at level 0 . For $n \geq 1$, denote by $a_{n}$ and $b_{n}$ the numbers of " 1 "-nodes and " 0 "-nodes at the $n$th level of the code tree, respectively. By the two type-I constraints, the following recursions hold:

$$
\left\{\begin{array}{l}
a_{n}=a_{n-1}+b_{n-1} \\
b_{n}=a_{n-1}
\end{array} \quad \text { for } n \geq 2 .\right.
$$

With the initial values of $a_{1}=1$ and $a_{2}=1$, it follows that $\left\{a_{n}\right\}_{n=1}^{\infty}$ is the renowned Fibonacci sequence [14], i.e., $a_{n}=a_{n-1}+a_{n-2}$ for $n \geq 3$. This result, together with the two type-II constraints, implies that the number of length- $n$ codewords is $\left|\mathcal{C}_{00}(n)\right|=a_{n}$ for $n \geq 1$, which according to the Fibonacci recursion is given by:

$$
a_{n}=\frac{\varphi^{n}-\bar{\varphi}^{n}}{\sqrt{5}},
$$

where $\varphi=\frac{1+\sqrt{5}}{2}$ is the Golden ratio and $\bar{\varphi}=\frac{1-\sqrt{5}}{2}$ is the Galois conjugate of $\varphi$ in number field $\mathbb{Q}(\sqrt{5})$. Thus, $\left|\mathcal{C}_{00}(n)\right|$ grows exponentially in $n$ with base $\varphi \approx 1.618$.

We can similarly examine the choice of UW $=01$ and draw the respective code tree in Fig. 2, where its type-I constraints become:

(C1) " 0 " can only be followed by " 0 ".

(C2) " 1 " can be followed by either " 0 " or " 1 ".

and no type-II constraints are required. We then obtain

$$
\left\{\begin{array}{l}
a_{n}=a_{n-1} \\
b_{n}=a_{n-1}+b_{n-1}
\end{array} \quad \text { for } n \geq 2,\right.
$$

and $\left|\mathcal{C}_{01}(n)\right|=a_{n}+b_{n}=n+1$. Although from Figs. 1 and 2 , taking $\mathrm{UW}=01$ seems to provide more codewords than taking $\mathrm{UW}=00$ at small $n$, the linear growth of $\left|\mathcal{C}_{01}(n)\right|$ with respect to codeword length $n$ suggests that such choice is not as good as the choice of UW $=00$ when $n$ is moderately large. 
The above two exemplified UWs point to an important fact that the best UW, which minimizes the average codeword length, depends on the code size required. Thus, the investigation of the efficiency of a UW may need to consider the transient superiority in addition to claiming the asymptotic winner.

In this paper, we provide efficient encoding and decoding algorithms for UDOOCs, and investigate their general combinatorial properties, in particular the enumeration of the number of codewords for any (finite) codeword length. Based on the obtained formula for $\left|\mathcal{C}_{\boldsymbol{k}}(n)\right|$, i.e., the number of length- $n$ codewords for a given UW $k$, the average codeword length of the optimal compression of a given source statistics using UDOOC can be computed. Classifications of UWs are followed, where two types of equivalences are specified, which are (exact) equivalence and asymptotic equivalence. UWs that are equivalent in the former sense are required to yield exactly the same minimum average codeword length for every source statistics, while asymptotic equivalence only dictates the UWs to result in the same asymptotic growth rate as codeword length approaches infinity. Enumeration of the number of asymptotic equivalent UW classes are then studied with the help of methodologies in [17] and [25]. Furthermore, three upper bounds on the average codeword length of UDOOCs are established. The first one is a general upper bound when only the largest probability of source symbols is given. The second upper bound refines the first one under the premise that the source entropy is additionally known. When both the largest and second largest probabilities of source symbols are present apart from the source entropy, the third upper bound can be used. Since these bounds are derived in terms of different techniques, actually none of the three bounds dominates the other two for all statistics. Comparison of these bounds for an English text with statistics from [36] and that with statistics from the book Alice's Adventures in Wonderland will be accordingly provided. The analysis on bounds of the average codeword length gives rise to two asymptotic bounds on ultimate per-letter average codeword length, one of which is tight for a certain choice of source statistics and UW, and the other of which leads to the achievability of the ultimate per-letter average codeword length to the source entropy rate when both the source block length for compression and UW length tend to infinity.

It may be of interest to note that the enumeration of the number of codewords, i.e., $c_{\boldsymbol{k}, n}=\left|\mathcal{C}_{\boldsymbol{k}}(n)\right|$, is actually obtained indirectly via the determination of an auxiliary quantity $s_{\boldsymbol{k}, n}$, which is the number of words satisfying the type-I constraints but not necessarily the type-II constraints. By utilizing the Goulden-Jackson cluster method [16], [20], [22], [23], [32], an explicit formula for $s_{\boldsymbol{k}, n}$ can be established. The desired enumeration formula for the number of length- $n$ UDOOC codewords is then obtained by proving that both the so-called linear constant coefficient difference equation (LCCDE) and the asymptotic growth rate of $s_{\boldsymbol{k}, n}$ and $c_{\boldsymbol{k}, n}$ are identical. We next show based on the obtained formula that the allzero UW has the largest asymptotic growth rate among all UWs of the same length, while the UW with the smallest growth rate is $00 \ldots 01$. Interestingly, the all-zero $\mathrm{UW}$ is often the one that yields the smallest $c_{\boldsymbol{k}, n}$ for small $n$, in contrast to UW $00 \ldots 01$, whose $c_{\boldsymbol{k}, n}$ tops all other UWs when $n$ is small. We afterwards demonstrate by using these two special UWs that the general encoding and decoding algorithms can be considerably simplified when further taking into consideration the structure of particular UWs. A side result from the enumeration of $c_{\boldsymbol{k}, n}$ is that for all UWs, the codeword growth rate of UDOOCs will tend to $|\mathbb{F}|=2$ as the length of the UW goes to infinity.

With regard to the compression performance of the proposed UDOOCs, numerical results show that when grouping three English letters as a block and separating the consecutive blocks by UWs, the UDOOCs with UW $=0001,0000,000001$ and 000000 can respectively reach the compression rates of 3.531, 4.089, 4.115, 4.709 bits per English letter (with the length of UWs included), where the source stream to be compressed is the book titled Alice's Adventures in Wonderland. In comparison with the first-order Huffman code, the secondorder Huffman code, the third-order Huffman $\operatorname{code}^{3}$ and the Lempel-Ziv code, which respectively achieve the compression rates of $3.940,3.585,3.226$ and 6.028 bits per English letter, the proposed UDOOCs can potentially result in comparable compression rate to the Huffman code under similar decoding complexity and yield a smaller average codeword length than that of the Lempel-Ziv code, thereby confirming the practicability of the scheme of separating OOC codewords by UWs.

In the literature, there are a number of publications on enumeration of words in a set that forbids the appearance of a specific pattern [8]-[12]. For example, Doroslova investigated the number of binary length- $n$ words, in which a specific subword like $1010 \ldots 10$ is not allowed [10]. He then extended the result to non-binary alphabet and forbidden subwords of length 3 [9], [12], and forbidden subwords of length 4 [11], as well as the so-called "good" forbidden subwords [8]. The analyses in [8]-[12] however depend on the specific structure of forbidden subwords considered, and no asymptotic examination is performed. On the other hand, algorithmic approaches have been devoted to a problem of similar (but not the same) kind, one of which is called the Goulden-Jackson clustering method [16], [20], [22], [23], [32].

Instead of enumerating the number of words internally without a forbidden pattern, some researchers investigate the inherent characteristic of such patterns. In this literature, Rivals and Rahmann [25] provide an algorithm to account for the number of overlaps ${ }^{4}$ for a given set of patterns, for which the definition will be later given in this paper for completeness (cf. Definition 4). Different from the algorithmic approach in [25], Guibas and Odlyzko established upper and lower bounds

\footnotetext{
${ }^{3} \mathrm{~A} k$ th-order Huffman code maps a block of $k$ source letters onto a variablelength codeword.

4In [17] and [25], the authors actually use a different name "autocorrelation" for "overlap" originated from [16]. Specifically, they define the autocorrelation $\boldsymbol{v}=v_{1} \cdots v_{L}$ of a binary length- $L$ string $\boldsymbol{u}=u_{1} \cdots u_{L}$ as a binary zero-one bit-stream of length $L$ such that $v_{i}=1$ if $i$ is a period of $\boldsymbol{u}$, where $i$ is said to be a period of $\boldsymbol{u}$ when $u_{j}=u_{i+j}$ for every $1 \leq j \leq L-i$. Since the term autocorrelation is extensively used in other literature ilke digital communications to illustrate similar but different conception, we adopt the name of "overlap" in this paper.
} 


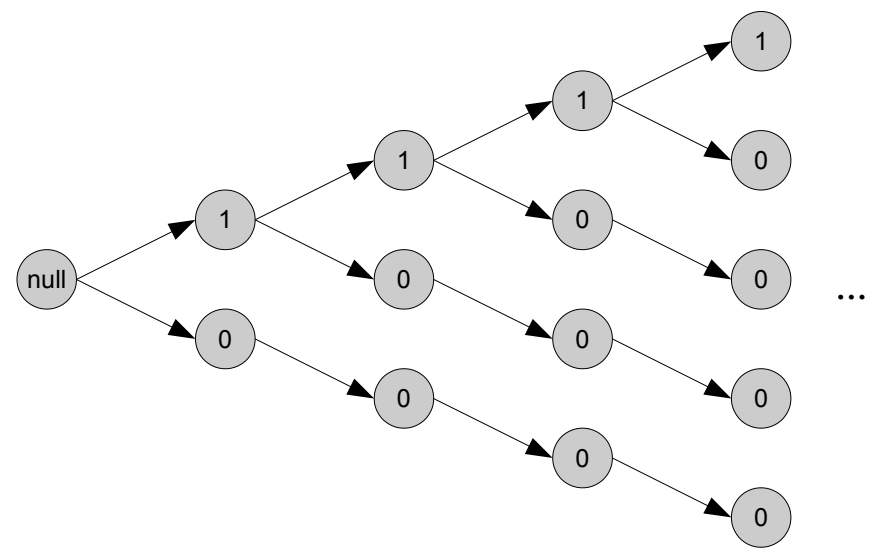

Fig. 2. UDOOC code tree for $\mathrm{UW}=01$.

for the number of overlaps when the length of the concerned pattern goes to infinity [17].

The rest of the paper is organized as follows. In Section II, construction of general UDOOCs is introduced. In Section III, combinatorial properties of UDOOCs, including the enumeration of the number of codewords, are derived. In Section IV, the encoding and decoding algorithms as well as bounds on average codeword length for general UDOOCs are provided and discussed. In Section V, numerical results on the compression performance of UDOOCs are presented. Conclusion is drawn in Section VI.

\section{Construction OF UDOOCs}

In the previous section, we have seen that the code tree of a UDOOC with $\mathrm{UW}=00$ (or $\mathrm{UW}=01$ ) is by far a useful tool for devising its properties. Along this line, we will provide a systematic construction of code tree for general UDOOC in this section. Specifically, a digraph [2] whose directional edges meet the type-I and type II constraints ${ }^{5}$ from the UW will be first introduced. By the digraph, the construction of a general UDOOC code tree as well as the determination of the growth rate of UDOOC codewords with respect to the codeword length will follow.

\section{A. Digraphs for UDOOCs}

Let $\boldsymbol{k}=k_{1} \ldots k_{L}$ be the chosen UW of length $L$. Denote by $G_{\boldsymbol{k}}=\left(V, E_{\boldsymbol{k}}\right)$ the digraph for the UDOOC with $\mathrm{UW}=\boldsymbol{k}$, where $V=\mathbb{F}^{L-1}$ is the set of all binary length- $(L-1)$ tuples, and $E_{\boldsymbol{k}}$ is the set of directional edges given by

$$
E_{\boldsymbol{k}}:=\left\{(\boldsymbol{i}, \boldsymbol{j}) \in V^{2}: i_{2}^{L-1}=j_{1}^{L-2} \text { and } i_{1} \boldsymbol{j} \neq \boldsymbol{k}\right\} .
$$

Here, we use the conventional shorthand $i_{s}^{t}=i_{s} i_{s+1} \ldots i_{t}$ to denote a binary string from index $s$ to index $t$, and the elements in $V$ are interchangeably denoted by either $i=i_{1} \ldots i_{L-1}$ or $i_{1}^{L-1}$, depending on whichever is more convenient.

\footnotetext{
${ }^{5}$ For clarity of its explanation, we introduce the so-called type-I and type-II constraints in Example 1. Listing these constraints for a general UW however may be tedious and less comprehensive. As will be seen from this section, these constraints can actually be absorbed into the construction of the digraph (See specifically Eq. (1)); hence, explicitly listing of constraints becomes of secondary necessity.
}



Fig. 3. Digraph $G_{010}$ for UW $\boldsymbol{k}=010$.

Define the $2^{L-1}$-by- $2^{L-1}$ adjacency matrix $\mathrm{A}_{\boldsymbol{k}}$ for the digraph $G_{\boldsymbol{k}}$ by putting its $(i+1, j+1)$ th entry as

$$
\left(\mathrm{A}_{\boldsymbol{k}}\right)_{i+1, j+1}= \begin{cases}1, & \text { if }(\boldsymbol{i}, \boldsymbol{j}) \in E_{\boldsymbol{k}} \\ 0, & \text { otherwise }\end{cases}
$$

where we abuse the notation by using $i$ (resp. $j$ ) to be the integer corresponding to binary representation of $i=$ $i_{1} \ldots i_{L-1}$ (resp. $\boldsymbol{j}=j_{1} \ldots j_{L-1}$ ) with the leftmost bit being the most significant bit. As an example, for $\boldsymbol{k}=010$, we have $V=\mathbb{F}^{2}=\{00,01,10,11\}$,

$$
\begin{aligned}
E_{010}=\{(00,00),(00,01),(01,11), & (10,00),(10,01),(11,10),(11,11)\},
\end{aligned}
$$

$G_{010}=\left(V, E_{010}\right)$ in Fig. 3, and

$$
\mathrm{A}_{010}=\left[\begin{array}{llll}
1 & 1 & 0 & 0 \\
0 & 0 & 0 & 1 \\
1 & 1 & 0 & 0 \\
0 & 0 & 1 & 1
\end{array}\right] .
$$

We remark that the adjacency matrix $A_{k}$ will be used for enumerating the number of UDOOC codewords in next section.

\section{B. Code Trees for UDOOCs}

Equipped with digraph $G_{k}$, constructing the code tree for the UDOOC with $\mathrm{UW}=\boldsymbol{k}$ becomes straightforward. Recall that a UDOOC codeword of length $n$ is a binary $n$-tuple $\boldsymbol{b}=b_{1} \ldots b_{n}$, satisfying that $\boldsymbol{k}$ is not an internal subword of the concatenated bit-stream $\boldsymbol{k} \boldsymbol{k} \boldsymbol{k}$. As such, the traversal of the digraph for constructing a UDOOC code tree should start from the vertex $k_{2}^{L} \in V$, which corresponds to the initial "null"node in the code tree. Next, a " 0 "-node at level 1 is generated if both $\left(k_{2}^{L}, j_{1}^{L-1}\right) \in E_{\boldsymbol{k}}$ and $j_{L-1}=0$ are satisfied. By the same rule, the "null"-node is followed by a "1"-node at level 1 if $\left(k_{2}^{L}, j_{1}^{L-1}\right) \in E_{\boldsymbol{k}}$ and $j_{L-1}=1$. We then move the current vertex to $j_{1}^{L-1}$ and draw a branch from " $j_{L-1}$ "-node at level 1 to a followup " 0 "-node (resp. " 1 "-node) at level 2 in the code tree if $\left(j_{1}^{L-1}, \ell_{1}^{L-1}\right) \in E_{k}$ and $\ell_{L-1}=0$ (resp. $\ell_{L-1}=1$ ). We move the current vertex again to $\ell_{1}^{L-1}$ and re-do the above procedure to generate the nodes in the next level. Repeating 
this process will complete the exploration of the nodes in the entire code tree.

Determination of the gray-shaded nodes that end a codeword can be done as follows. Since $\boldsymbol{k}$ cannot be an internal subword of $\boldsymbol{k} \boldsymbol{b} \boldsymbol{k}$, a node should be gray-shaded if it is immediately followed by a sequence of offspring nodes with their binary marks equal to $k_{1} \ldots k_{L-1}$. The construction of the UDOOC code tree is accordingly finished.

As an example, we continue from the exemplified UW $\boldsymbol{k}=010$ with digraph $G_{\boldsymbol{k}}$ in Fig. 3 and explore its respective UDOOC code tree in Fig. 4 by following the previously mentioned procedure. By starting from the vertex $k_{2}^{3}=10$ that corresponds to the "null"-node, two succeeding nodes are generated since both $(10,00)$ and $(10,01)$ are in $E_{010}$ (cf. Fig. 4). Now from vertex 00 that corresponds to the " 0 "node at level 1, we can reach either vertex 00 or vertex 01 in one transition; hence, both " 0 "-node and " 1 "-node are the succeeding nodes to the " 0 "-node at level 1 . However, since vertex 01 can only walk to vertex 11 in one transition, the " 1 "node at level 1 has only one succeeding node with mark " 1 ." Continuing this process then exhausts all the nodes in the code tree in Fig. 4. Next, all nodes that are followed by $k_{1} k_{2}=01$ in sequence in the code tree are gray-shaded. The construction of the code tree for the UDOOC with UW $\boldsymbol{k}=010$ is then completed.

We end this section by giving the type-I and type-II constraints for the exemplified code tree as follows.

- Type-I constraints:

(C1) " 0 " can be followed by either " 0 " or " 1 ".

(C2) " 1 " can be followed by " 0 " only when the node prior to this " 1 "-node is not a " 0 "-node.

- Type-II constraints:

(C3) The first two bits of a UDOOC codeword cannot be "10."

(C4) The last two bits of a UDOOC codeword cannot be "01."

Note that with these constraints (in particular (C4)), one can also perform the node-shading step by first gray-shading all the nodes in the code tree, and then unshade those that end with " 01 " (in addition to the " 1 "-node at level 1 for this specific UW). Nevertheless, it may be tedious to perform the nodeunshading for a general UW. For example, when UW $\boldsymbol{k}=$ 01001, all nodes that end a codeword $b_{1}^{n}$, satisfying either $b_{n-3} b_{n-2} b_{n-1} b_{n} k_{1}=\boldsymbol{k}$ or $b_{n-2} b_{n-1} b_{n} k_{1} k_{2}=\boldsymbol{k}$, should be unshaded. This confirms the superiority of constructing the UDOOC code tree in terms of the digraph over analyzing the explicit listing of constraints from the adopted UW that are perhaps convenient only for some special UWs.

\section{COMBinatorial PROPERTIES OF UDOOCS}

\section{A. The Determination of $\left|\mathcal{C}_{\boldsymbol{k}}(n)\right|$}

In this subsection, we will see that the conception of digraph $G_{k}$, in particular its respective adjacency matrix $A_{k}$, can lead to a formula for the number of length- $n$ codewords, i.e., $c_{\boldsymbol{k}, n}=$ $\left|\mathcal{C}_{\boldsymbol{k}}(n)\right|$.

In accordance with the fact that the traversal of the digraph for constructing a UDOOC code tree should start from vertex

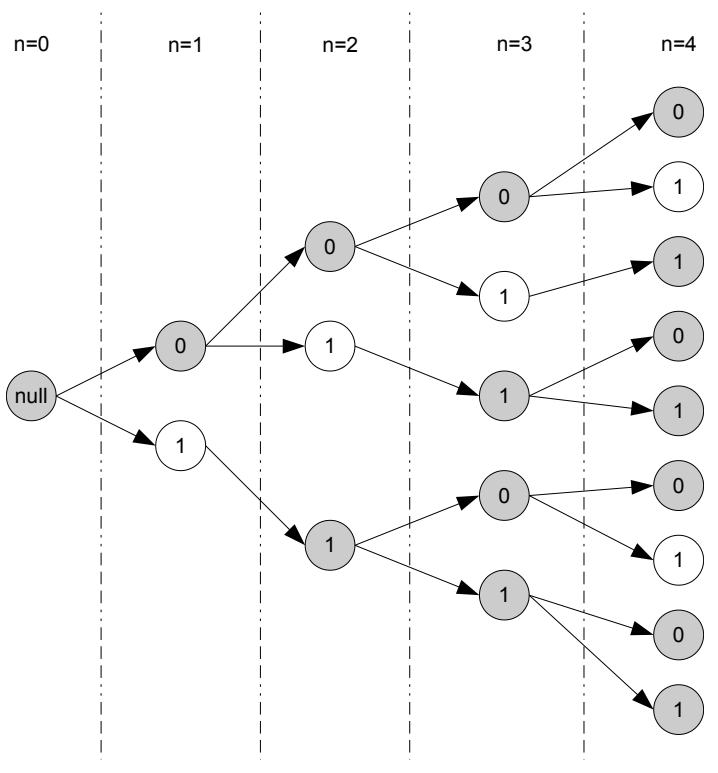

Fig. 4. Code tree for the UDOOC with UW $k=010$.

$k_{2}^{L} \in V$, we define a length- $2^{L-1}$ initial vector as $\left(\underline{x}_{\boldsymbol{k}}\right)_{j+1}=1$ if integer $j$ has the binary representation $k_{2}^{L}$, and $\left(\underline{x}_{\boldsymbol{k}}\right)_{j+1}=0$, otherwise, for $0 \leq j<2^{L-1}$. It then follows that the $(\ell+1)$ th entry of row vector $x_{k}^{\top} \mathrm{A}_{k}^{n}$ gives the number of length- $n$ walks that end at vertex $\ell$ on digraph $G_{k}$, where "T" denotes the vector/matrix transpose operation, and $\ell=\ell_{1} \ldots \ell_{L-1}$ is the binary representation of integer index $\ell$.

However, not every length- $n$ walk produces a codeword. Notably, some nodes on the code tree will be gray-shaded and some will not. Recall that $\boldsymbol{k}$ cannot be an internal subword of $\boldsymbol{k} \boldsymbol{b} \boldsymbol{k}$ if $\boldsymbol{b}=b_{1} b_{2} \ldots b_{n}$ is a codeword. This implies that $\boldsymbol{b}$ is a length- $n$ codeword if, and only if, the vertex sequence $k_{2}^{L}, k_{3}^{L} b_{1}, k_{4}^{L} b_{1}^{2}, \ldots, b_{n} k_{1}^{L-2}, k_{1}^{L-1}$ is a valid walk of length $n+L-1$ on digraph $G_{\boldsymbol{k}}$. As a result, the number of length- $n$ codewords equals the number of length- $(n+L-1)$ walks from vertex $k_{2}^{L}$ to vertex $k_{1}^{L-1}$ on digraph $G_{\boldsymbol{k}}$. Following the above discussion, we define the length- $2^{L-1}$ ending vector $\underline{y}_{\boldsymbol{k}}$ as $\left(\underline{y}_{\boldsymbol{k}}\right)_{j+1}=1$ if integer $j$ has the binary representation $k_{1}^{L-1}$, and $\left(\underline{y}_{k}\right)_{j+1}=0$, otherwise, for $0 \leq j<2^{L-1}$. Then, the number of length- $n$ codewords is given by

$$
c_{\boldsymbol{k}, n}:=\left|\mathcal{C}_{\boldsymbol{k}}(n)\right|=\underline{x}_{\boldsymbol{k}}^{\top} \mathrm{A}_{\boldsymbol{k}}^{n+L-1} \underline{y}_{\boldsymbol{k}} .
$$

\section{B. Equivalence among $U W s$}

Two UWs that result in the same minimum average codeword length for every source statistics should be considered equivalent. This leads to the following definition.

Definition 2: Two UWs $\boldsymbol{k}$ and $\boldsymbol{k}^{\prime}$ are said to be equivalent, denoted by $\boldsymbol{k} \equiv \boldsymbol{k}^{\prime}$, if the numbers of their length- $n$ codewords in the corresponding UDOOCs are the same for all $n$, i.e.,

$$
c_{\boldsymbol{k}, n}=c_{\boldsymbol{k}^{\prime}, n} \text { for all } n \geq 0 \text {. }
$$

By this definition, UDOOCs associated with equivalent UWs have the same number of codewords in every code tree level; hence they achieve the same minimum average 
codeword length in the lossless compression of a sequence of source letters. This equivalence relation allows us to focus only on one UW in every equivalent class. It is however hard to exhaust and identify all equivalent classes of UWs of arbitrary length. Instead, we will introduce a less restrictive notion of asymptotic equivalence when the asymptotic compression rate of UDOOCs is concerned, and derive the number of all asymptotically equivalent classes of UWs in Section III-E.

Some properties about the (exact) equivalence of UWs are given below.

Proposition 1 (Equivalence in order reversing): UW $\boldsymbol{k}^{\prime}=$ $k_{L} \ldots k_{1}$ is equivalent to UW $\boldsymbol{k}=k_{1} k_{2} \ldots k_{L}$.

Proof: It follows simply from that $\boldsymbol{b}=b_{1} b_{2} \ldots b_{n} \in \mathcal{C}_{\boldsymbol{k}}$ if, and only if, $\boldsymbol{b}^{\prime}=b_{n} b_{n-1} \ldots b_{1} \in \mathcal{C}_{\boldsymbol{k}^{\prime}}$.

Proposition 2 (Equivalence in binary complement): If $\overline{\boldsymbol{k}}$ is the bit-wise binary complement of $\boldsymbol{k}$, then $\bar{k}$ and $\boldsymbol{k}$ are equivalent.

Proof: It is a consequence of the fact that the concatenated bit-stream $\boldsymbol{k} \boldsymbol{b} \boldsymbol{k}$ contains $\boldsymbol{k}$ as an internal subword if, and only if, the binary complement $\overline{\boldsymbol{k} \boldsymbol{b} \boldsymbol{k}}$ of $\boldsymbol{k} \boldsymbol{b} \boldsymbol{k}$ contains $\overline{\boldsymbol{k}}$ as an internal subword.

From Propositions 1 and 2, it can be verified that there are at most four equivalent classes for UWs of length $L=4$. Representative UWs for these four equivalent classes are 0000, 0001, 0100 and 0101, respectively.

\section{Growth Rates of UDOOCs}

In this subsection, we investigate the asymptotic growth rate of UDOOCs, of which the definition is given below.

Definition 3: Given UW $\boldsymbol{k}$, the asymptotic growth rate of the resulting UDOOC is defined as

$$
g_{\boldsymbol{k}}:=\lim _{n \rightarrow \infty} \frac{c_{\boldsymbol{k}, n+1}}{c_{\boldsymbol{k}, n}} .
$$

By its definition, the asymptotic growth rate of a UDOOC indicates how fast the number of codewords grows as $n$ increases.

It is obvious that $g_{k} \leq 2$ for all UWs because the upper bound of 2 is the growth rate for unconstrained binary sequences of length $n$. In addition, the limit in (5) must exist since it can be inferred from enumerative combinatorics [28], and also from algebraic graph theory [4], that $g_{k}$ is the largest eigenvalue of adjacency matrix $A_{k}$. In the next proposition, we show that the largest eigenvalue of adjacency matrix $A_{k}$ is unique for all UWs but $\boldsymbol{k}=01$.

Proposition 3 (Uniqueness of the largest eigenvalue of $\mathrm{A}_{\boldsymbol{k}}$ ): For any UW $\boldsymbol{k}$ of length $L \geq 2$ except $\boldsymbol{k}=01$, the largest eigenvalue of adjacency matrix $A_{k}$ is unique and is real.

Proof: By Perron-Frobenius theorem [15] [19], the largest eigenvalue of adjacency matrix $\mathrm{A}_{\boldsymbol{k}}$ is unique and real with algebraic multiplicity equal to 1 if $G_{\boldsymbol{k}}$ is a strongly connected diagrph. Thus, we only need to show that $G_{k}$ is a strongly connected digraph except for $\boldsymbol{k}=01$.

We then argue that $G_{\boldsymbol{k}}$ is a strongly connected diagrph when $L \geq 3$ as follows. According to the definition of $E_{k}$ in (1),

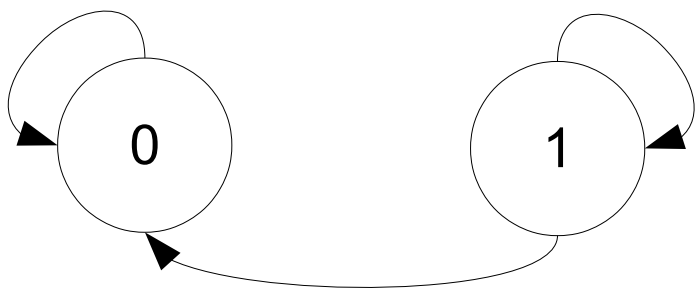

Fig. 5. Digraph $G_{\boldsymbol{k}}$ for UW $\boldsymbol{k}=01$.

the only situation that a vertex may not be strongly connected to other vertex is when $\boldsymbol{j}=k_{2} k_{3} \cdots k_{L}$. This however cannot happen when $L \geq 3$ because vertex $\bar{k}_{1} k_{2} \cdots k_{L-1}$ will connect strongly to $k_{2} k_{3} \cdots k_{L}$. The proof is completed after verifying the two cases for $L=2$, i.e., $G_{00}$ is strongly connected but $G_{01}$ is not.

The digraph for $\boldsymbol{k}=01$ is plotted in Fig. 5. It clearly indicates that there is no directed path from vertex 0 to vertex 1. In fact, the algebraic multiplicity of the largest eigenvalue 1 of $A_{01}$ is two.

By the standard technique of using an indeterminate $z$ in enumerative combinatorics, we can enumerate the numbers $c_{\boldsymbol{k}, n}$ as

$$
\begin{aligned}
\sum_{n=0}^{\infty} c_{\boldsymbol{k}, n} z^{n} & =\sum_{n=0}^{\infty} \underline{x}_{\boldsymbol{k}}^{\top} \mathrm{A}_{\boldsymbol{k}}^{n+L-1} \underline{y}_{\boldsymbol{k}} z^{n} \\
& =\underline{x}_{\boldsymbol{k}}^{\top}\left(\sum_{n=0}^{\infty} \mathrm{A}_{\boldsymbol{k}}^{n} z^{n}\right) \mathrm{A}_{\boldsymbol{k}}^{L-1} \underline{y}_{\boldsymbol{k}} \\
& =\underline{x}_{\boldsymbol{k}}^{\top}\left(\mathrm{I}-\mathrm{A}_{\boldsymbol{k}} z\right)^{-1} \mathrm{~A}_{\boldsymbol{k}}^{L-1} \underline{y}_{\boldsymbol{k}} \\
& =\frac{\underline{x}_{\boldsymbol{k}}^{\top} \operatorname{adj}\left(\mathrm{I}-\mathrm{A}_{\boldsymbol{k}} z\right) \mathrm{A}_{\boldsymbol{k}}^{L-1} \underline{y}_{\boldsymbol{k}}}{\operatorname{det}\left(\mathrm{I}-\mathrm{A}_{\boldsymbol{k}} z\right)},
\end{aligned}
$$

where the first equality follows from (3) and I denotes the identity matrix of proper size. Equation (6) then implies that $\operatorname{det}\left(\mathrm{I}-\mathrm{A}_{\boldsymbol{k}} z\right)$ can give a linear recursion of $c_{\boldsymbol{k}, n}$ in the form of a linear constant coefficient difference equation (LCCDE).

Now let $\lambda_{1}, \ldots, \lambda_{m}$ be distinct nonzero eigenvalues of adjacency matrix $\mathrm{A}_{\boldsymbol{k}}$ with algebraic multiplicities $e_{1}, \ldots, e_{m}$, respectively, where we assume with no loss of generality that $\left|\lambda_{1}\right| \geq \cdots \geq\left|\lambda_{m}\right|$. In terms of the standard technique of partial fraction for rational functions, we can rewrite (6) as

$$
\frac{\underline{x}_{\boldsymbol{k}}^{\top} \operatorname{adj}\left(\mathrm{I}-\mathrm{A}_{\boldsymbol{k}} z\right) \mathrm{A}_{\boldsymbol{k}}^{L-1} \underline{y}_{\boldsymbol{k}}}{\operatorname{det}\left(\mathrm{I}-\mathrm{A}_{\boldsymbol{k}} z\right)}=\sum_{i=1}^{m} \frac{p_{i}(z)}{\left(1-\lambda_{i} z\right)^{e_{i}}}
$$

for some polynomials $p_{i}(z)$. The next step is expectantly to rewrite the righ-hand-side (RHS) of (7) as a power series of indeterminate $z$ in order to recover the actual values of $c_{\boldsymbol{k}, n}$ for all $n$. As an example, this can be done by

$$
\frac{1}{\left(1-\lambda_{i} z\right)^{e_{i}}}=\sum_{n=0}^{\infty}\left(\begin{array}{c}
n+e_{i}-1 \\
n
\end{array}\right) \lambda_{i}^{n} z^{n}
$$


which holds for all $|z|<\min _{1 \leq i \leq m} \frac{1}{\left|\lambda_{i}\right|}$.

Although the asymptotic growth rate $g_{k}$ equals exactly the largest eigenvalue of adjacency matrix $A_{k}$, it is in general difficult to find a closed-form expression for this value without a proper reshaping of adjacency matrix $A_{k}$. Another approach is to consider the following set for $n \geq L$,

$$
\mathcal{S}_{\boldsymbol{k}}(n):=\left\{\boldsymbol{b} \in \mathbb{F}^{n}: \boldsymbol{k} \text { is not a subword of } \boldsymbol{b}\right\},
$$

which, in a way, defines the set of distinct length- $n$ walks on digraph $G_{\boldsymbol{k}}$. Denoting $s_{\boldsymbol{k}, n}:=\left|\mathcal{S}_{\boldsymbol{k}}(n)\right|$ and by an argument similar to (6), one can easily show that

$$
\sum_{n=0}^{\infty} s_{\boldsymbol{k}, n} z^{n}=\sum_{n=0}^{L-1} 2^{n} z^{n}+z^{L} \frac{\underline{\mathbf{1}}^{\top} \operatorname{adj}\left(\mathrm{I}-\mathrm{A}_{\boldsymbol{k}} z\right) \underline{\mathbf{1}}}{\operatorname{det}\left(\mathrm{I}-\mathrm{A}_{\boldsymbol{k}} z\right)}
$$

where $\underline{1}$ is the all-one column vector of appropriate length. Equation (9) then implies that the enumeration of $s_{\boldsymbol{k}, n}$ also depends upon the polynomial $\operatorname{det}\left(\mathrm{I}-\mathrm{A}_{\boldsymbol{k}} z\right)$ as $c_{\boldsymbol{k}, n}$ does. Based on this observation, we can infer and prove that $s_{\boldsymbol{k}, n}$ has the same asymptotic growth rate as $c_{\boldsymbol{k}, n}$. We summarize this important inference in the proposition below, while the proof will be relegated to the next subsection.

Proposition 4: For any UW $\boldsymbol{k}$, sequences $\left\{c_{\boldsymbol{k}, n}\right\}_{n=0}^{\infty}$ and $\left\{s_{\boldsymbol{k}, n}\right\}_{n=0}^{\infty}$ have the same asymptotic growth rate, i.e.,

$$
g_{k}=\mathfrak{g}_{k},
$$

where

$$
\mathfrak{g}_{\boldsymbol{k}}:=\lim _{n \rightarrow \infty} \frac{s_{\boldsymbol{k}, n+1}}{s_{\boldsymbol{k}, n}} .
$$

Notably, in order to distinguish the asymptotic growth rate of $s_{\boldsymbol{k}, n}$ from that of $c_{\boldsymbol{k}, n}$, a different font $\mathfrak{g}_{\boldsymbol{k}}$ is used to denote the asymptotic growth rate of $s_{\boldsymbol{k}, n}$.

\section{Enumeration of $s_{\boldsymbol{k}, n}$}

Enumerating $s_{\boldsymbol{k}, n}$ turns out to be easier than enumerating $c_{\boldsymbol{k}, n}$ due to that there is lesser number of constraints on the sequences in $\mathcal{S}_{\boldsymbol{k}}(n)$. It can be done by an approach similar to the Goulden-Jackson clustering method [23]. Before delivering the main theorems, we define the overlap function and overlap vector of a binary stream $k$ as follows.

Definition 4: For a given $\boldsymbol{k}$ of length $L$, its overlap function is defined as

$$
r_{\boldsymbol{k}}(i):= \begin{cases}1 & \text { if } k_{i+1}^{L}=k_{1}^{L-i} \text { and } 0 \leq i \leq L-1 \\ 0 & \text { otherwise. }\end{cases}
$$

Furthermore, we define its length- $L$ overlap vector as $\left(\underline{r}_{\boldsymbol{k}}\right)_{j}=$ $r_{\boldsymbol{k}}(j-1)$ for $j=1 \ldots L$.

Theorem 1: For a length- $L \mathrm{UW} \boldsymbol{k}$ with overlap function $r_{\boldsymbol{k}}(i)$,

$$
\sum_{n \geq 0} s_{\boldsymbol{k}, n} z^{n}=\frac{1+\sum_{i=1}^{L-1} r_{\boldsymbol{k}}(i) z^{i}}{(1-2 z)\left(1+\sum_{i=1}^{L-1} r_{\boldsymbol{k}}(i) z^{i}\right)+z^{L}} .
$$

Moreover, let $h_{\boldsymbol{k}}(z)$ denote the denominator of (11), i.e.,

$$
h_{\boldsymbol{k}}(z)=(1-2 z)\left(1+\sum_{i=1}^{L-1} r_{\boldsymbol{k}}(i) z^{i}\right)+z^{L}
$$

Then

$$
h_{\boldsymbol{k}}(z)=\operatorname{det}\left(\mathrm{I}-\mathrm{A}_{\boldsymbol{k}} z\right)
$$

where $\mathrm{A}_{\boldsymbol{k}}$ is the adjacency matrix associated with digraph $G_{\boldsymbol{k}}$.

Proof: The result (11) follows from the Goulden-Jackson clustering method [23]. For completeness, a simplified proof to this claim is provided in Appendix A. To establish the second claim, i.e., (13), we combine (9) and (11) to give

$$
\frac{1+\sum_{i=1}^{L-1} r_{\boldsymbol{k}}(i) z^{i}}{(1-2 z)\left(1+\sum_{i=1}^{L-1} r_{\boldsymbol{k}}(i) z^{i}\right)+z^{L}}=\frac{f(z)}{\operatorname{det}\left(\mathrm{I}-\mathrm{A}_{\boldsymbol{k}} z\right)}
$$

for some polynomial $f(z)$. Notice that the left-hand-side (LHS) is an irreducible rational function in $z$. Furthermore, Proposition 10 in Appendix B shows $\operatorname{deg} \operatorname{det}\left(\mathrm{I}-\mathrm{A}_{\boldsymbol{k}} z\right)=L$. These then imply that

$$
\operatorname{det}\left(\mathrm{I}-\mathrm{A}_{\boldsymbol{k}} z\right)=h_{\boldsymbol{k}}(z)
$$

and

$$
f(z)=1+\sum_{i=1}^{L-1} r_{\boldsymbol{k}}(i) z^{i}
$$

(13) is thus established.

The next example illustrates the usage of the above theorem to the target result of Proposition 4.

Example 2: Consider the case of $\boldsymbol{k}=000$. Then from (10), the corresponding overlap function is

$$
r_{000}(i)= \begin{cases}1, & i=0,1,2, \\ 0, & \text { otherwise. }\end{cases}
$$

Substituting the above into (11), we obtain

$$
\sum_{n \geq 0} s_{000, n} z^{n}=\frac{1+z+z^{2}}{1-z-z^{2}-z^{3}} .
$$

By regarding the above as an LCCDE, we conclude that the sequence $s_{000, n}$ satisfies the following recursion for all $n \geq 0$ : $s_{000, n}=s_{000, n-1}+s_{000, n-2}+s_{000, n-3}+\delta_{n}+\delta_{n-1}+\delta_{n-2}$, where

$$
\delta_{n}= \begin{cases}1, & n=0 \\ 0, & \text { otherwise }\end{cases}
$$

is the Kronecker delta function.

Equipped with Theorem 1, we are now ready to prove Proposition 4.

Proof of Proposition 4: From the proof of Theorem 1, we have seen that the enumeration of $s_{\boldsymbol{k}, n}$ is given by the following irreducible rational function

$$
\sum_{n=0}^{\infty} s_{\boldsymbol{k}, n} z^{n}=\frac{1+\sum_{i=1}^{L-1} r_{\boldsymbol{k}}(i) z^{i}}{h_{\boldsymbol{k}}(z)}
$$

where $h_{\boldsymbol{k}}(z)$ is the denominator of (11) and is given by (12). Hence, it follows from the standard partial fraction technique and Proposition 3 that

$$
\mathfrak{g}_{k}=\max \left\{|u|^{-1}: h_{\boldsymbol{k}}(u)=0, u \in \mathbb{C}\right\},
$$


where $\mathbb{C}$ is the set of complex numbers. Next, noticing that the function $h_{\boldsymbol{k}}(z)$, i.e., $\operatorname{det}\left(\mathrm{I}-\mathrm{A}_{\boldsymbol{k}} z\right)$, also appears as the denominator of the enumeration function for $c_{\boldsymbol{k}, n}$ (cf. (6)), we get

$$
g_{\boldsymbol{k}} \leq \max \left\{|u|^{-1}: h_{\boldsymbol{k}}(u)=0, u \in \mathbb{C}\right\}
$$

since the rational function in (6) could be reducible. This shows $g_{\boldsymbol{k}} \leq \mathfrak{g}_{\boldsymbol{k}}$.

To prove $g_{\boldsymbol{k}} \geq \mathfrak{g}_{\boldsymbol{k}}$ (which then implies $g_{\boldsymbol{k}}=\mathfrak{g}_{\boldsymbol{k}}$ ), it suffices to show that $c_{\boldsymbol{k}, n+2} \geq s_{\boldsymbol{k}, n}$ for $n \geq L$. This can be done by substantiating that for any $\boldsymbol{b} \in \mathcal{S}_{\boldsymbol{k}}(n)$, there exist a prefix bit $p$ and a suffix bit $q$, where $p, q \in \mathbb{F}$, such that $p \boldsymbol{b} q \in \mathcal{C}_{\boldsymbol{k}}(n+2)$.

Using the prove-by-contradiction argument, we first assume that $\boldsymbol{k}$ is an internal subword of both $\boldsymbol{k} p \boldsymbol{b}$ and $\boldsymbol{k} \bar{p} \boldsymbol{b}$, where $\bar{p}=1-p$. This assumption, together with $\boldsymbol{b} \in \mathcal{S}_{\boldsymbol{k}}(n)$, implies the existence of indices $1<i<L+2$ and $1<j<L+2$ such that

$$
\underbrace{k_{i} \cdots k_{L} p b_{1} \cdots b_{i-2}}_{=\boldsymbol{a}}=\underbrace{k_{j} \cdots k_{L} \bar{p} b_{1} \cdots b_{j-2}}_{=\tilde{\boldsymbol{a}}}=\boldsymbol{k}
$$

where we abuse the notations to let

$$
\boldsymbol{a}= \begin{cases}k_{i} \cdots k_{L} p, & \text { if } i=2 \\ k_{i} \cdots k_{L} p b_{1} \cdots b_{i-2}, & \text { if } 2<i<L+1 \\ p b_{1} \cdots b_{i-2}, & \text { if } i=L+1\end{cases}
$$

and similar notational abuse is applied to $\tilde{\boldsymbol{a}}$ and $j$. Assume without loss of generality that $i<j$. Then, the sums of the last $(j-1)$ bits of $\boldsymbol{a}$ and $\tilde{\boldsymbol{a}}$ must equal, i.e.,

$k_{L-(j-i-1)}+\cdots+k_{L}+p+b_{1}+\cdots+b_{i-2}=\bar{p}+b_{1}+\cdots+b_{j-2}$.

Canceling out common terms at both sides gives

$$
k_{L-(j-i-1)}+\cdots+k_{L}+p=\bar{p}+b_{i-1}+\cdots+b_{j-2} .
$$

Note again that $\tilde{\boldsymbol{a}}=\boldsymbol{k}$; hence, substituting $b_{(j-2)-\ell}$ by $k_{L-\ell}$ for $\ell=0,1, \cdots, j-i-1$ in (16) gives $p=\bar{p}$, which contradicts the assumption that $\bar{p}=1-p$.

For the suffix bit $q$, we again assume to the contrary that there exist indices $i$ and $j$, satisfying $n+1-L<i<j<n+2$, such that

$$
\underbrace{b_{i} \cdots b_{n} q k_{1} \cdots k_{L-n+i-2}}_{=\boldsymbol{d}}=\underbrace{b_{j} \cdots b_{n} \bar{q} k_{1} \cdots k_{L-n+j-2}}_{=\tilde{\boldsymbol{d}}}=\boldsymbol{k}
$$

After canceling out common terms in the respective sums of the first $(n+2-i)$ bits of $\boldsymbol{d}$ and $\tilde{\boldsymbol{d}}$, we obtain

$$
b_{i}+\cdots+b_{j-1}+q=\bar{q}+k_{1}+\cdots+k_{j-i} .
$$

Since $\boldsymbol{d}=\boldsymbol{k}$, the above implies $q=\bar{q}$, which again leads to a contradiction.

One application of the result $h_{\boldsymbol{k}}(z)=\operatorname{det}\left(\mathrm{I}-\mathrm{A}_{\boldsymbol{k}} z\right)$ in Theorem 1 is to obtain a recursion formula for $c_{\boldsymbol{k}, n}$, i.e., an LCCDE for $c_{\boldsymbol{k}, n}$. This is provided in the next corollary.
Corollary 1: For a length- $L$ UW $\boldsymbol{k}$ with overlap function $r_{\boldsymbol{k}}(i)$, let $c_{\boldsymbol{k}, n}$ be the number of length- $n$ codewords in the UDOOC $\mathcal{C}_{k}$ defined as before. Then, for $n \geq L$,

$c_{\boldsymbol{k}, n}=\left[\sum_{i=1}^{L-1} r_{\boldsymbol{k}}(i)\left(2 c_{\boldsymbol{k}, n-i-1}-c_{\boldsymbol{k}, n-i}\right)\right]+2 c_{\boldsymbol{k}, n-1}-c_{\boldsymbol{k}, n-L}$.

Proof: To prove (18), we first note that the characteristic polynomial for $\mathrm{A}_{\boldsymbol{k}}$ is given by

$$
\begin{aligned}
\chi_{\mathrm{A}_{\boldsymbol{k}}}(z) & =\operatorname{det}\left(z \mathrm{I}-\mathrm{A}_{\boldsymbol{k}}\right) \\
& =z^{2^{L-1}} h_{\boldsymbol{k}}(1 / z) \\
& =z^{2^{L-1}-L}\left(z^{L} h_{\boldsymbol{k}}(1 / z)\right)
\end{aligned}
$$

where $z^{L} h_{\boldsymbol{k}}(1 / z)$ is a polynomial with degree

$$
\operatorname{deg} h_{\boldsymbol{k}}(z)=\operatorname{deg} \operatorname{det}\left(\mathrm{I}-\mathrm{A}_{\boldsymbol{k}} z\right)=L .
$$

Denote

$$
m=\min \left\{p>0: \operatorname{Nullity}\left(\mathrm{A}_{k}^{p}\right)=2^{L-1}-L\right\},
$$

where $\operatorname{Nullity}()$ indicates the dimension of the null space of the square matrix inside parentheses. By Cayley-Hamilton Theorem [18], the following polynomial

$$
\begin{aligned}
\mu_{\boldsymbol{k}}(z) & :=z^{m}\left(z^{L} h_{\boldsymbol{k}}(1 / z)\right) \\
= & z^{m}\left(z^{L}-2 z^{L-1}+\sum_{i=1}^{L-1} r_{\boldsymbol{k}}(i) z^{L-i-1}(z-2)+1\right)
\end{aligned}
$$

is an annihilating polynomial for $A_{k}$. We shall remark that $\mu_{\boldsymbol{k}}(z)$ needs not to be the minimal polynomial for $\mathrm{A}_{\boldsymbol{k}}$. Plugging (20) into (3) yields that for $n-1 \geq \max \{m, L-1\}$, we have

$$
\begin{aligned}
& c_{\boldsymbol{k}, n} \\
& =\underline{x}_{\boldsymbol{k}}^{\top} \mathrm{A}_{\boldsymbol{k}}^{n+L-1} \underline{y}_{\boldsymbol{k}} \\
& =\underline{x}_{\boldsymbol{k}}^{\top} \mathrm{A}_{\boldsymbol{k}}^{n-1-m} \mathrm{~A}_{\boldsymbol{k}}^{m+L} \underline{y}_{\boldsymbol{k}} \\
& =\underline{x}_{\boldsymbol{k}}^{\top} \mathrm{A}_{\boldsymbol{k}}^{n-1}\left[2 \mathrm{~A}_{\boldsymbol{k}}^{L-1}+\sum_{i=1}^{L-1} r_{\boldsymbol{k}}(i)\left(2 \mathrm{~A}_{\boldsymbol{k}}^{L-i-1}-\mathrm{A}_{\boldsymbol{k}}^{L-i}\right)-\mathrm{I}\right] \underline{y}_{\boldsymbol{k}} \\
& =\left[\sum_{i=1}^{L-1} r_{\boldsymbol{k}}(i)\left(2 c_{\boldsymbol{k}, n-i-1}-c_{\boldsymbol{k}, n-i}\right)\right]+2 c_{\boldsymbol{k}, n-1}-c_{\boldsymbol{k}, n-L},
\end{aligned}
$$

where the condition of $n-1 \geq \max \{m, L-1\}$ follows from i) $n-1-m \geq 0$ such that (21) holds, and ii) $n-1 \geq L-1$ such that the last term of the RHS of (22) represents $c_{\boldsymbol{k}, n-L}$. Finally, since $\operatorname{rank}\left(\mathrm{A}_{k}^{p}\right) \leq L$ for $p=L-1$ (see Proposition 10), we have $m \leq L-1$, which immediately gives $\max \{m, L-1\}=$ $L-1$. The proof is thus completed.

So far, we learn that $c_{\boldsymbol{k}, n}$ and $s_{\boldsymbol{k}, n}$ have the same asymptotic growth rate, and both of their enumerations depend on $\operatorname{det}(\mathrm{I}-$ $\left.\mathrm{A}_{\boldsymbol{k}} z\right)$. Below we will use $s_{\boldsymbol{k}, n}$ to determine the asymptotic growth rates corresponding to two specific UWs, $\boldsymbol{a}=0 \ldots 00$ and $\boldsymbol{b}=0 \ldots 01$. We then proceed to show that $\boldsymbol{a}$ has the largest growth rate among all UWs of the same length, while the smallest growth rate is resulted when $\mathrm{UW}=\boldsymbol{b}$. 
Theorem 2: Among all UWs of the same length, the allzero UW has the largest growth rate, while UW $0 \ldots 01$ achieves the smallest.

Proof: For notational convenience, we set $\boldsymbol{a}=0 \ldots 0$ and $\boldsymbol{b}=0 \ldots 01$. For $\mathrm{UW}=\boldsymbol{a}$, it can be verified from (10) and (11) that

$$
h_{\boldsymbol{a}}(z)=1-\sum_{i=1}^{L} z^{i}
$$

and hence the sequence of $\left\{s_{\boldsymbol{a}, n}\right\}_{n=1}^{\infty}$ satisfies the following recursion:

$$
s_{\boldsymbol{a}, n}=\sum_{i=1}^{L} s_{\boldsymbol{a}, n-i} \quad \text { for } n \geq L .
$$

Similarly, we have $h_{\boldsymbol{b}}(z)=1-2 z+z^{L}$, and therefore,

$$
s_{\boldsymbol{b}, n}=2 s_{\boldsymbol{b}, n-1}-s_{\boldsymbol{b}, n-L} \quad \text { for } n \geq L .
$$

For general UW $\boldsymbol{k}$ of length $L$, (11) gives the following recursion for $n \geq L$

$s_{\boldsymbol{k}, n}=\sum_{i=1}^{L-1}\left(2 s_{\boldsymbol{k}, n-i-1}-s_{\boldsymbol{k}, n-i}\right) r_{\boldsymbol{k}}(i)+2 s_{\boldsymbol{k}, n-1}-s_{\boldsymbol{k}, n-L}$.

Note that $r_{\boldsymbol{k}}(i) \in\{0,1\}$ by definition, and $2 s_{\boldsymbol{k}, m-1} \geq s_{\boldsymbol{k}, m}$ for all $m$. From (24), the following bounds hold for any UW $\boldsymbol{k}$ with $n \geq L$ :

$$
2 s_{\boldsymbol{k}, n-1}-s_{\boldsymbol{k}, n-L} \leq s_{\boldsymbol{k}, n} \leq \sum_{i=1}^{L} s_{\boldsymbol{k}, n-i}
$$

where the lower and upper bounds are respectively obtained by replacing all $r_{\boldsymbol{k}}(i)$ in (24) by 0 and 1 . In particular, $s_{\boldsymbol{k}, n}$ equals the upper bound in (25) when $\boldsymbol{k}=\boldsymbol{a}=00 \cdots 0$, and the lower bound is achieved when $\boldsymbol{k}$ is $\boldsymbol{b}=00 \cdots 01$. By dividing all terms in (25) by $s_{\boldsymbol{k}, n-1}$ and taking $n \rightarrow \infty$, we obtain

$$
2-g_{\boldsymbol{k}}^{-(L-1)} \leq g_{\boldsymbol{k}} \leq 1+g_{\boldsymbol{k}}^{-1}+\cdots+g_{\boldsymbol{k}}^{-L+1}
$$

To prove our claim that $g_{\boldsymbol{a}}$ is the largest and $g_{\boldsymbol{b}}$ is the smallest among all $g_{\boldsymbol{k}}$, we first assume to the contrary that there exists $\hat{\boldsymbol{k}}$ with $g_{\hat{\boldsymbol{k}}}>g_{\boldsymbol{a}}$. Substituting this into (26) leads to the following contradiction

$$
g_{\hat{\boldsymbol{k}}} \stackrel{(\mathrm{i})}{<} \sum_{i=1}^{L} g_{\boldsymbol{a}}^{-i+1} \stackrel{(\mathrm{ii})}{=} g_{\boldsymbol{a}}
$$

where (i) holds because $g_{\hat{\boldsymbol{k}}}^{-1}<g_{\boldsymbol{a}}^{-1}$ by assumption and (ii) is valid because $g_{\boldsymbol{a}}^{-1}$ is a zero of $h_{\boldsymbol{a}}(z)$ given in (23).

To show $g_{b}$ achieves the minimum, again assume to the contrary that there exists $\hat{\boldsymbol{k}}$ such that $g_{\hat{\boldsymbol{k}}}<g_{\boldsymbol{b}}$. Note from (26) that

$0 \leq g_{\hat{\boldsymbol{k}}}-2+g_{\hat{\boldsymbol{k}}}^{-(L-1)}=\left(1-g_{\hat{\boldsymbol{k}}}\right)\left(g_{\hat{\boldsymbol{k}}}^{-L+1}+\cdots+g_{\hat{\boldsymbol{k}}}^{-1}-1\right)$.

Although $g_{\boldsymbol{k}} \geq 1$ in general, we claim in this case $g_{\hat{\boldsymbol{k}}}>1$. For otherwise, that $h_{\hat{\boldsymbol{k}}}\left(z=g_{\hat{\boldsymbol{k}}}^{-1}=1\right)=0$ according to (12) implies that $r_{\hat{\boldsymbol{k}}}(i)=0$ for all $i$; hence, $h_{\hat{\boldsymbol{k}}}(z)=h_{\boldsymbol{b}}(z)$ and $g_{\hat{k}}=g_{\boldsymbol{b}}$, a contradiction. Now with $1<g_{\hat{\boldsymbol{k}}}<g_{\boldsymbol{b}}$, the following series of inequalities lead to the desired contradiction:

$$
g_{\boldsymbol{b}} \stackrel{(\mathrm{i})}{=} g_{\boldsymbol{b}}^{-L+2}+\cdots+1 \stackrel{\text { (ii) }}{<} g_{\hat{\boldsymbol{k}}}^{-L+2}+\cdots+1 \stackrel{\text { (iii) }}{\leq} g_{\hat{\boldsymbol{k}}},
$$

where (i) follows from $h_{\boldsymbol{b}}\left(z=g_{\boldsymbol{b}}^{-1}\right)=0$ and $g_{\boldsymbol{b}}>1$, (ii) holds because $g_{\boldsymbol{b}}^{-1}<g_{\hat{\boldsymbol{k}}}^{-1}$, and (iii) is due to (27) and $g_{\hat{\boldsymbol{k}}}>1$.

Using a similar technique in the proof of Theorem 2, we can further devise a general upper bound and a general lower bound for $g_{\boldsymbol{k}}$ that hold for any $\boldsymbol{k}$.

Theorem 3: For any UW $k$ of length $L \geq 2$, the asymptotic growth rate $g_{k}$ satisfies

$$
2-2^{-(L-2)} \leq g_{k} \leq 2-2^{-L} .
$$

Proof: It is straightforward to see $s_{\boldsymbol{k}, n-1} \leq s_{\boldsymbol{k}, n} \leq$ $2 s_{\boldsymbol{k}, n-1}$ and hence $1 \leq g_{\boldsymbol{k}} \leq 2$.

To prove the upper bound, we assume without loss of generality that $g_{k}>1$ since the upper bound trivially holds when $g_{k}=1$. We then derive

$$
g_{k}-1=g_{\boldsymbol{k}}\left(1-g_{\boldsymbol{k}}^{-1}\right) \stackrel{\text { (i) }}{\leq} 1-g_{\boldsymbol{k}}^{-L} \stackrel{\text { (ii) }}{\leq} 1-2^{-L},
$$

where (i) follows from multiplying both sides of the second inequality in (26) by $\left(1-g_{k}^{-1}\right)$ with the fact $g_{\boldsymbol{k}}>1$, and (ii) holds since $g_{k} \leq 2$.

To establish the lower bound, we use the following series of inequalities:

$$
\begin{aligned}
g_{\boldsymbol{k}}\left(1-g_{\boldsymbol{k}}^{-1}\right) & =g_{\boldsymbol{k}}-1 \\
& \stackrel{\text { (i) }}{\geq}-g_{\boldsymbol{k}}^{-(L-1)} \\
& =\left(1-g_{\boldsymbol{k}}^{-1}\right)\left(1+g_{\boldsymbol{k}}^{-1}+g_{\boldsymbol{k}}^{-2}+\cdots+g_{\boldsymbol{k}}^{-(L-2)}\right) \\
& \stackrel{\text { (ii) }}{\geq}\left(1-g_{\boldsymbol{k}}^{-1}\right)\left(1+2^{-1}+2^{-2}+\cdots+2^{-(L-2)}\right) \\
& =\left(1-g_{\boldsymbol{k}}^{-1}\right)\left(2-2^{-(L-2)}\right),
\end{aligned}
$$

where (i) is from the first inequality in (26), and (ii) holds because $g_{k} \leq 2$. Equipped with (29), we next distinguish two cases to complete the proof.

1) When $L=2$, the lower bound is trivially valid and is actually achieved by taking $\mathrm{UW}=01$ as $g_{01}=1$ is the multiplicative inverse of the smallest zero of polynomial $h_{01}(z)=1-2 z+z^{2}=(1-z)^{2}$.

2) For $L>2$, it suffices to show $g_{k}>1$. Assume to the contrary that there exists $k$ of length $L>2$ such that $g_{\boldsymbol{k}}=1$. By $h_{\boldsymbol{k}}\left(z=g_{\boldsymbol{k}}^{-1}=1\right)=0$ and (12), we have $r_{\boldsymbol{k}}(i)=0$ for all $i$ and hence $h_{\boldsymbol{k}}(z)=1-2 z+z^{L}$. Since $g_{k}$ is the multiplicative inverse of the smallest zero of $h_{\boldsymbol{k}}(z)$, the absolute values of all the remaining zeros of $h_{\boldsymbol{k}}(z)$, say $\lambda_{1}, \ldots, \lambda_{L-1}$, must be strictly larger than 1 . It then follows from the splitting of $h_{\boldsymbol{k}}(z)$, i.e.,

$$
h_{\boldsymbol{k}}(z)=(z-1) \prod_{i=1}^{L-1}\left(z-\lambda_{i}\right)
$$


the constant term of $h_{\boldsymbol{k}}(z)$ must have absolute value $\prod_{i=1}^{L-1}\left|\lambda_{i}\right|>1$, contradicting to the fact that the constant term in polynomial $h_{\boldsymbol{k}}(z)=1-2 z+z^{L}$ is 1 .

Theorem 3 provides concrete explicit expressions for both upper and lower bounds on $g_{\boldsymbol{k}}$. Although the bounds are asymptotically tight and well approximate the true $g_{k}$ for moderately large $L$, they are not sharp in general. We can actually refine them using Theorem 2 and obtain that $g_{\boldsymbol{b}} \leq g_{\boldsymbol{k}} \leq g_{\boldsymbol{a}}$, where from the proof of Theorem 2, we have

$$
g_{\boldsymbol{a}}=\max \left\{|t|: h_{\boldsymbol{a}}\left(z=t^{-1}\right)=0, t \in \mathbb{C}\right\}
$$

and

$$
g_{\boldsymbol{b}}=\max \left\{|t|: h_{\boldsymbol{b}}\left(z=t^{-1}\right)=0, t \in \mathbb{C}\right\} .
$$

The determination of $g_{\boldsymbol{a}}$ and $g_{\boldsymbol{b}}$ can be done via finding the largest $|s|$ and $|t|, 0 \neq s, t \in \mathbb{C}$, such that $h_{\boldsymbol{a}}\left(s^{-1}\right)=0$ and $h_{\boldsymbol{b}}\left(t^{-1}\right)=0$, respectively. By noting that

$$
\begin{aligned}
(z-1)\left[z^{L} h_{\boldsymbol{a}}\left(z^{-1}\right)\right] & =(z-1)\left(z^{L}-z^{L-1}-\cdots-1\right) \\
& =z^{L+1}-2 z^{L}+1
\end{aligned}
$$

and

$$
z^{L} h_{\boldsymbol{b}}\left(z^{-1}\right)=z^{L}-2 z^{L-1}+1
$$

we conclude the following corollary.

Corollary 2: Let $\boldsymbol{a}=0 \ldots 0$ and $\boldsymbol{b}=0 \ldots 01$ be binary streams of length $L$. Then for any $\boldsymbol{k}$ of the same length to $\boldsymbol{a}$ and $\boldsymbol{b}$,

$$
g_{b} \leq g_{k} \leq g_{a}
$$

In addition, $g_{\boldsymbol{a}}=\alpha_{L+1}$ and $g_{\boldsymbol{b}}=\alpha_{L}$, where

$$
\alpha_{L}:=\max \left\{|t|: t^{L}-2 t^{L-1}+1=0, t \in \mathbb{C}\right\} .
$$

In particular, we have $\alpha_{L} \approx 2-2^{-L+1}$ for large $L$.

Based on Theorem 3, the following corollary is immediate by taking $L$ to infinity.

Corollary 3: For any UW $\boldsymbol{k}$ of length $L$, the asymptotic growth rate of the corresponding UDOOC approaches 2 as $L \rightarrow \infty$, i.e.,

$$
\lim _{L \rightarrow \infty} g_{k}=2
$$

In Table I, we illustrate the asymptotic growth rates of UDOOCs for UWs $\boldsymbol{a}$ and $\boldsymbol{b}$ with lengths up to 8. Also shown are the bounds in Theorem 3. It is seen that for moderately large $L$, all UDOOCs have roughly the same asymptotic growth rate, and hence are about the same good in terms of compressing sources of large size. Furthermore, having $g_{k} \rightarrow 2$ as $L \rightarrow \infty$ means that for very large $L$, UDOOCs can have asymptotic growth rates comparable to the unconstrained OOC, whose asymptotic growth rate equals 2 .
TABLE I

THE ASYMPTOTIC GROWTH RATES FOR UWS $\boldsymbol{a}$ AND $\boldsymbol{b}$ AND THE BOUNDS IN THEOREM 3 WITH VARIOUS $L$

\begin{tabular}{c|ccccccc}
\hline$L$ & 2 & 3 & 4 & 5 & 6 & 7 & 8 \\
\hline $2-2^{-L}$ & 1.75 & 1.875 & 1.938 & 1.969 & 1.984 & 1.992 & 1.996 \\
\hline$g_{a}$ & 1.618 & 1.839 & 1.928 & 1.966 & 1.984 & 1.992 & 1.996 \\
\hline$g_{b}$ & 1 & 1.618 & 1.839 & 1.928 & 1.966 & 1.984 & 1.992 \\
\hline $2-2^{-(L-2)}$ & 1 & 1.5 & 1.75 & 1.875 & 1.938 & 1.969 & 1.984 \\
\hline
\end{tabular}

\section{E. Asymptotic Equivalence}

After presenting the results on asymptotic growth rates, we proceed to define the asymptotic equivalence for UWs and show that the number of asymptotic equivalent UW classes is upper bounded by the number of different overlap vectors in Definition 4.

Definition 5: Two UWs $k$ and $\boldsymbol{k}^{\prime}$ are said to be asymptotically equivalent, denoted by $\boldsymbol{k} \stackrel{\text { a.e. }}{\equiv} \boldsymbol{k}^{\prime}$, if they have the same growth rate, i.e., $g_{\boldsymbol{k}}=g_{\boldsymbol{k}^{\prime}}$.

Following the definition, we have the next proposition.

Proposition 5: Fix the length $L$ of UWs, and denote by $N_{L}$ the number of all possible overlap vectors of length $L$, i.e., $N_{L}=\left|\left\{\underline{r}_{\boldsymbol{k}}: \boldsymbol{k} \in \mathbb{F}^{L}\right\}\right|$. Then, the number of asymptotically equivalent UW classes is upper-bounded by $N_{L}$.

Proof: Since the growth rate of $s_{\boldsymbol{k}, n}$ is given by $\max \left\{|t|: h_{\boldsymbol{k}}\left(z=t^{-1}\right)=0, t \in \mathbb{C}\right\}$, in which the polynomial $h_{\boldsymbol{k}}(z)$, defined in (12), is completely determined by the respective overlap vector $\underline{r}_{k}$. As two different polynomials $h_{\boldsymbol{k}}(z)$ and $h_{\boldsymbol{k}^{\prime}}(z)$, resulting respectively from two different overlap vectors $\underline{r}_{\boldsymbol{k}}$ and $\underline{r}_{\boldsymbol{k}^{\prime}}$, could yield the same growth rate, the number of distinct asymptotic growth rates of $s_{\boldsymbol{k}, n}$ for various $\boldsymbol{k}$ must be upper-bounded by $N_{L}$. The proof is then completed after invoking the result from Proposition 4 that $s_{\boldsymbol{k}, n}$ and $c_{\boldsymbol{k}, n}$ have the same growth rate.

One may find the number of asymptotically equivalent UW classes by a brutal force algorithm when $L$ is small. With the help of Proposition 5, an efficient algorithm for its upper bound $N_{L}$ is available in [25], in which $\underline{\boldsymbol{r}}_{\boldsymbol{k}}$ is regarded as (auto)correlations of a string. Values of $N_{L}$ for various $L$ are accordingly listed in Table II. This table shows the trend, as being pointed out in [17], that $\ln N_{L}$ grows at the speed of $(\ln L)^{2}$, or specifically,

$$
\frac{1}{2 \ln 2} \leq \liminf _{L \rightarrow \infty} \frac{\ln N_{L}}{(\ln L)^{2}} \leq \limsup _{L \rightarrow \infty} \frac{\ln N_{L}}{(\ln L)^{2}} \leq \frac{1}{2 \ln \frac{3}{2}}
$$

TABLE II

$N_{L}$ VALUES FOR VARIOUS $L$. IT IS STATED IN [25] THAT THE LOWER ASYMPTOTIC BOUND $1 /(2 \ln (2)) \approx 0.72$ IN (30) ONLY HOLDS FOR VERY LARGE $L$; HENCE, THIS LOWER BOUND IS NOT VALID FOR $L \leq 13$ IN THIS TABLE .

\begin{tabular}{c|ccccccccccccc}
\hline$L$ & 1 & 2 & 3 & 4 & 5 & 6 & 7 & 8 & 9 & 10 & 11 & 12 & 13 \\
\hline$N_{L}$ & 1 & 2 & 3 & 4 & 6 & 8 & 10 & 13 & 17 & 21 & 27 & 30 & 37 \\
\hline$\frac{\ln N_{L}}{(\ln L)^{2}}$ & - & 1.44 & .91 & .72 & .69 & .65 & .61 & .59 & 59 & .57 & .57 & .55 & .55 \\
\hline
\end{tabular}




\section{Encoding And Decoding Algorithms of UDOOCS}

In this section, the encoding and decoding algorithms of UDOOCs are presented. Also provided are upper bounds for the averaged codeword length of the resulting UDOOC.

Denote by $\mathcal{U}=\left\{u_{1}, u_{2}, \cdots, u_{M}\right\}$ the source alphabet of size $M$ to be encoded. Assume without loss of generality that $p_{1} \geq p_{2} \geq \cdots \geq p_{M}$, where $p_{i}$ is the probability of occurrence for source symbol $u_{i}$.

Then, an optimal lossless source coding scheme for UDOOCs associated with UW $\boldsymbol{k}$ should assign codewords of shorter lengths to messages with higher probabilities and reserve longer codewords for less likely messages. By following this principle, the encoding mapping $\phi_{\boldsymbol{k}}$ from $\mathcal{U}$ to $\mathcal{C}_{\boldsymbol{k}}$ should satisfy $\ell\left(\phi_{\boldsymbol{k}}\left(u_{i}\right)\right) \leq \ell\left(\phi_{\boldsymbol{k}}\left(u_{j}\right)\right)$ whenever $i \leq j$, where $\ell\left(\phi_{\boldsymbol{k}}\left(u_{i}\right)\right)$ denotes the length of bit stream $\phi_{\boldsymbol{k}}\left(u_{i}\right)$. The coding system thus requires an ordering of the words in $\mathcal{C}_{\boldsymbol{k}}$ according to their lengths. This can be achieved in terms of the recurrence equation for $c_{\boldsymbol{k}, n}$ (for example, (22)). As such, $\phi_{\boldsymbol{k}}\left(u_{1}\right)$ must be the null word, and the mapping $\phi_{\boldsymbol{k}}$ must always form a bijection mapping between $\left\{u_{i}: F_{\boldsymbol{k}, n-1}<i \leq F_{\boldsymbol{k}, n}\right\}$ and $\mathcal{C}_{\boldsymbol{k}}(n)$ for every integer $n \geq 1$, where

$$
F_{\boldsymbol{k}, n}:= \begin{cases}\sum_{i=0}^{n} c_{\boldsymbol{k}, i}, & \text { if } n \geq 0, \\ 0, & \text { otherwise. }\end{cases}
$$

This optimal assignment results in average codeword length:

$$
L_{\boldsymbol{k}}=\ell(\boldsymbol{k})+\sum_{i=1}^{M} p_{i} \cdot \ell\left(\phi_{\boldsymbol{k}}\left(u_{i}\right)\right),
$$

where the first term $\ell(\boldsymbol{k})$ accounts for the insertion of UW $\boldsymbol{k}$ to separate adjacent codewords.

\section{A. Upper Bounds on Average Codeword Length of UDOOCs}

The average codeword length $L_{k}$ is clearly a function of the source distributions and does not in general exhibit a closed-form formula. In order to understand the general compression performance of UDOOCs, three upper bounds on $L_{k}$ are established in this subsection. The first upper bound is applicable to the situation when the largest probability $p_{1}$ of source symbols is given. Other than $p_{1}$, the second upper bound additionally requires the knowledge of the source entropy. When both the largest and second largest probabilities (i.e., $p_{1}$ and $p_{2}$ ) of source symbols are present apart from the source entropy, the third upper bound can be used. Note that the third upper bound holds for all UWs and requires no knowledge about $\boldsymbol{k}$; therefore, one might predict that the third upper bound could be looser than the other two. Experiments using English text from Alice's Adventures in Wonderland however indicate that such an intuitive prediction is not always valid. Nevertheless, the second upper bound is better than the first one in most cases we have examined. Details are given below.

Proposition 6 (The first upper bound on $L_{\boldsymbol{k}}$ ): For UW $\boldsymbol{k}$ of length $L$, the average codeword length $L_{\boldsymbol{k}}$ is upper-bounded as follows:

$$
L_{k} \leq L+\left(1-p_{1}\right) N_{k}
$$

where $N_{k}$ is the smallest integer such that $F_{k, N_{k}} \geq M$.

Proof: It can be derived from (32) and $\ell\left(\phi_{\boldsymbol{k}}\left(u_{1}\right)\right)=0$ that

$$
\begin{aligned}
L_{\boldsymbol{k}} & =L+\sum_{i=2}^{M} p_{i} \ell\left(\phi_{\boldsymbol{k}}\left(u_{i}\right)\right) \\
& \leq L+\sum_{i=2}^{M} p_{i} \ell\left(\phi_{\boldsymbol{k}}\left(u_{M}\right)\right) \\
& =L+\left(1-p_{1}\right) N_{\boldsymbol{k}} .
\end{aligned}
$$

Proposition 7 (The second upper bound on $L_{k}$ ): Suppose $g_{k}>1$. Then

$$
\begin{aligned}
L_{\boldsymbol{k}} \leq L+\frac{\mathrm{H}(\mathcal{U})+p_{1} \log _{2}\left(p_{1}\right)}{\log _{2}\left(g_{\boldsymbol{k}}\right)} & \\
& +\left(1-p_{1}\right)\left(1-\log _{g_{\boldsymbol{k}}}\left(K_{\boldsymbol{k}}\right)\right)
\end{aligned}
$$

where $\mathrm{H}(\mathcal{U})=\sum_{i=1}^{M} p_{i} \log _{2}\left(1 / p_{i}\right)$ is the source entropy with units in bits, $K_{k}$ is a constant given by

$$
K_{\boldsymbol{k}}=\min \left\{g_{\boldsymbol{k}}^{1-n_{i}} F_{\boldsymbol{k}, n_{i}-1}: i=2, \cdots, M\right\},
$$

and $n_{i}$ is the smallest integer satisfying $F_{\boldsymbol{k}, n_{i}} \geq i$.

Proof: From the definitions of $n_{i}$ and $K_{k}$ we have

$$
K_{\boldsymbol{k}} g_{\boldsymbol{k}}^{n_{i}-1} \leq F_{\boldsymbol{k}, n_{i}-1}<i \leq \frac{1}{p_{i}}
$$

where the last inequality follows from that $p_{i} \leq \frac{1}{i}$ for $1 \leq$ $i \leq M$ as $p_{1} \geq p_{2} \geq \cdots \geq p_{M}$. By $g_{\boldsymbol{k}}>1$ the above implies

$$
n_{i} \leq 1-\log _{g_{k}}\left(K_{\boldsymbol{k}} p_{i}\right)=1-\log _{g_{k}}\left(p_{i}\right)-\log _{g_{k}}\left(K_{\boldsymbol{k}}\right) .
$$

Note that $\ell\left(\phi_{\boldsymbol{k}}\left(u_{i}\right)\right) \leq n_{i}$ by the property of optimal lossless compression function $\phi_{\boldsymbol{k}}$. Consequently, we have

$$
\begin{aligned}
L_{\boldsymbol{k}} & =L+\sum_{i=2}^{M} p_{i} \ell\left(\phi_{\boldsymbol{k}}\left(u_{i}\right)\right) \\
& \leq L+\sum_{i=2}^{M} p_{i} n_{i} \\
& \leq L+\sum_{i=2}^{M} p_{i}\left(1-\log _{g_{\boldsymbol{k}}}\left(p_{i}\right)-\log _{g_{\boldsymbol{k}}}\left(K_{\boldsymbol{k}}\right)\right) \\
& =L-\sum_{i=2}^{M} p_{i} \log _{g_{\boldsymbol{k}}}\left(p_{i}\right)+\sum_{i=2}^{M} p_{i}\left(1-\log _{g_{\boldsymbol{k}}}\left(K_{\boldsymbol{k}}\right)\right) \\
& =L+\frac{\mathrm{H}(\mathcal{U})+p_{1} \log _{g_{\boldsymbol{k}}}\left(p_{1}\right)}{\log _{2}\left(g_{\boldsymbol{k}}\right)}+\left(1-p_{1}\right)\left(1-\log _{g_{\boldsymbol{k}}} K_{\boldsymbol{k}}\right)
\end{aligned}
$$

The previous two upper bounds require the computations of either $N_{k}$, or $g_{k}$ and $K_{k}$; hence, they are functions of UW $k$. Next we provide a simple third upper bound that holds universally for all UWs.

Proposition 8 (The third upper bound on $L_{k}$ ): For UW $\boldsymbol{k}$ of length $L>2$,

$$
L_{\boldsymbol{k}} \leq L+\frac{\mathrm{H}(\mathcal{U})+p_{1} \log _{2}\left(p_{1}\right)+p_{2} \log _{2}\left(p_{2}\right)}{\log _{2}\left(2-2^{2-L}\right)}
$$




$$
+\left(2-2 p_{1}-p_{2}\right) .
$$

Proof: First, we claim that

$$
c_{\boldsymbol{k}, n} \geq 2^{n-2} \text { for } 2 \leq n \leq L+1 .
$$

This claim can be established by showing that for any binary sequence $\boldsymbol{b}=b_{1} \ldots b_{m} \in \mathbb{F}^{m}$, where $0 \leq m=n-2 \leq L-1$, there exist a prefix bit $p$ and a suffix bit $q$, where $p, q \in \mathbb{F}$, such that $\boldsymbol{k}$ is not an internal subword of $\boldsymbol{k} p \boldsymbol{b} q \boldsymbol{k}$. This can be done in two steps: i) there exists $q \in \mathbb{F}$ such that $\boldsymbol{k}$ is not a subword of $\boldsymbol{u}:=\boldsymbol{b} q k_{1}^{L-1}$, and ii) there exists $p \in \mathbb{F}$ such that $\boldsymbol{k}$ is not an internal subword of $\boldsymbol{k} p \boldsymbol{u}$.

Because the first step trivially holds when $m=0$, we only need to focus on the case of $m>0$. Utilizing the prove-bycontradiction argument, we suppose that $\boldsymbol{k}$ is a subword of both $\boldsymbol{b} q k_{1}^{L-1}$ and $\boldsymbol{b} \bar{q} k_{1}^{L-1}$, where $\bar{q}=1-q$. This implies the existence of indices $1 \leq i<j \leq m+1$ such that

$\underbrace{b_{i} \cdots b_{m} q k_{1} \cdots k_{L-m+i-2}}_{=\boldsymbol{d}}=\underbrace{b_{j} \cdots b_{m} \bar{q} k_{1} \cdots k_{L-m+j-2}}_{=\tilde{\boldsymbol{d}}}=\boldsymbol{k}$

where we abuse the notations to let

$$
\boldsymbol{d}= \begin{cases}\boldsymbol{b} q k_{1} \cdots k_{L-m-1}, & \text { if } i=1 \\ b_{i} \cdots b_{m} q k_{1} \cdots k_{L-m+i-2}, & \text { if } 1<i<m+1 \\ q k_{1} \cdots k_{L-1}, & \text { if } i=m+1\end{cases}
$$

and similar notational abuse is applied to $\tilde{\boldsymbol{d}}$ and $j$. After canceling out common terms in the respective sums of the first $(m+2-i)$ bits of $\boldsymbol{d}$ and $\tilde{\boldsymbol{d}}$, we obtain

$$
b_{i}+\cdots+b_{j-1}+q=\bar{q}+k_{1}+\cdots+k_{j-i} .
$$

Since $\boldsymbol{d}=\boldsymbol{k}$, the above then implies $q=\bar{q}$, which leads to a contradiction. The validity of the first step is verified.

After verifying $\boldsymbol{u}=\boldsymbol{b} q k_{1}^{L-1} \in \mathcal{S}_{\boldsymbol{k}}(m+L)$, we can follow the proof of Proposition 4 to confirm the second step (See the paragraph regarding (14) and (15)). The claim in (37) is thus validated. Note that the equality in (37) holds when $\boldsymbol{k}$ is all-zero or all-one.

Next, we note also from the proof of Proposition 4 that $c_{\boldsymbol{k}, n+2} \geq s_{\boldsymbol{k}, n}$ for $n \geq L$. Since $s_{\boldsymbol{k}, L}=2^{L}-1$, we immediately have $c_{\boldsymbol{k}, L+2} \geq 2^{L}-1$. On the other hand, we can obtain from (25) that ${ }^{6}$

$$
\frac{s_{\boldsymbol{k}, n}}{s_{\boldsymbol{k}, n-1}} \geq 2-2^{2-L} \text { for } n \geq L .
$$

This concludes:

$$
c_{\boldsymbol{k}, n} \geq \begin{cases}1, & \text { if } 0 \leq n \leq 1, \\ 2^{n-2}, & \text { if } 2 \leq n \leq L+1, \\ \left(2-2^{2-L}\right)^{n-L-2}\left(2^{L}-1\right), & \text { if } n \geq L+2,\end{cases}
$$

where $c_{\boldsymbol{k}, 0}=1$ because $\mathcal{C}_{\boldsymbol{k}}(0)$ contains only the null codeword, and $c_{\boldsymbol{k}, 1} \geq 1$ can be verified again by that $\boldsymbol{k}$ cannot be the internal subword of both $\boldsymbol{k} p \boldsymbol{k}$ and $\boldsymbol{k} \bar{p} \boldsymbol{k} .{ }^{7}$ The lower bound (39) then indicates that if $2^{L}-1 \geq\left(2-2^{2-L}\right)^{L}$ for $L>2$, we can immediately have the following exponential lower bound for $c_{k, n}$, i.e.,

$$
c_{k, n} \geq \begin{cases}1, & \text { if } 0 \leq n \leq 1 \\ \left(2-2^{2-L}\right)^{n-2}, & \text { if } n \geq 2 .\end{cases}
$$

A stronger claim of $2^{L}-1 \geq\left(2-2^{2-L}\right)^{L}$ for $L>0$ simply follows from

$$
2^{L}-1-\left(2-2^{2-L}\right)^{L}>2^{L}-1-2^{L-1}\left(2-2^{2-L}\right)=1 .
$$

Hence, codeword lengths of the optimal UDOOC code must satisfy: ${ }^{8}$

$$
\ell\left(\phi_{\boldsymbol{k}}\left(u_{i}\right)\right) \leq \log _{2-2^{2-L}}(i)+2 \text { for } i \geq 3 .
$$

Consequently,

$$
L_{\boldsymbol{k}}=L+\sum_{i=2}^{M} p_{i} \ell\left(\phi_{\boldsymbol{k}}\left(u_{i}\right)\right)
$$

\footnotetext{
${ }^{6}$ We can prove (38) by induction. Extending the definition of $\mathcal{S}_{\boldsymbol{k}}(n)$ in (8), we obtain that $s_{\boldsymbol{k}, n}=2^{n}$ for $0 \leq n<L$. This implies

$$
\frac{s_{\boldsymbol{k}, L}}{s_{\boldsymbol{k}, L-1}}=\frac{2^{L}-1}{2^{L-1}}=2-2^{1-L} \geq 2-2^{2-L}
$$

and

$$
\frac{s_{\boldsymbol{k}, m}}{s_{\boldsymbol{k}, m-1}}=\frac{2^{m}}{2^{m-1}}=2 \geq 2-2^{2-L} \text { for all } 1 \leq m<L .
$$

Now we suppose that for some $n \geq L$ fixed, (38) is true for all $1 \leq m \leq n$, i.e.,

$$
\frac{s_{\boldsymbol{k}, m}}{s_{\boldsymbol{k}, m-1}} \geq 2-2^{2-L} \text { for all } 1 \leq m \leq n .
$$

Then, we derive by (25) that

$$
\begin{aligned}
\frac{s_{\boldsymbol{k}, n+1}}{s_{\boldsymbol{k}, n}} & \geq 2-\frac{s_{\boldsymbol{k}, n-L+1}}{s_{\boldsymbol{k}, n}} \geq 2-\frac{s_{\boldsymbol{k}, n-L+1}}{s_{\boldsymbol{k}, n-L+1}\left(2-2^{2-L}\right)^{L-1}} \\
& =2-\left(2-2^{2-L}\right)^{1-L} \geq 2-2^{2-L} .
\end{aligned}
$$
}

This completes the proof of (38).

${ }^{7}$ If it were not true, then there exist indices $i$ and $j, 2 \leq i<j \leq L+1$, such that $\boldsymbol{k}=k_{i} \cdots k_{L} p k_{1} \cdots k_{i-2}=k_{j} \cdots k_{L} \bar{p} k_{1} \cdots k_{j-2}$; hence, $p-k_{i}=$ $\bar{p}-k_{j}$ with $k_{i}=k_{j}=k_{1}$. The desired contradiction is obtained.

${ }^{8} \mathrm{By}(31)$ and (40), we have that for $i \geq 3$ and $n_{i}=\ell\left(\phi_{\boldsymbol{k}}\left(u_{i}\right)\right)$,

$$
i>F_{\boldsymbol{k}, n_{i}-1}=\sum_{t=0}^{n_{i}-1} c_{\boldsymbol{k}, t} \geq 2+\frac{\left(2-2^{2-L}\right)^{n_{i}-2}-1}{1-2^{2-L}}
$$

which implies $\log _{2-2^{2-L}}\left[(i-2)\left(1-2^{2-L}\right)+1\right]+2>n_{i}=\ell\left(\phi_{\boldsymbol{k}}\left(u_{i}\right)\right)$. Since $(i-2)\left(1-2^{2-L}\right)+1 \leq i$ for $i \geq 2-2^{L-2}$, we obtain

$\ell\left(\phi_{\boldsymbol{k}}\left(u_{i}\right)\right)<\log _{2-2^{2-L}}\left[(i-2)\left(1-2^{2-L}\right)+1\right]+2 \leq \log _{2-2^{2-L}}(i)+2$. 


$$
\begin{aligned}
& =L+p_{2}+\sum_{i=3}^{M} p_{i} \ell\left(\phi_{\boldsymbol{k}}\left(u_{i}\right)\right) \\
& \leq \quad L+p_{2}+\sum_{i=3}^{M} p_{i}\left(\log _{2-2^{2-L}}(i)+2\right) \\
& =\quad L+2-2 p_{1}-p_{2}+\sum_{i=3}^{M} p_{i} \log _{2-2^{2-L}}(i) \\
& \leq \quad L+2-2 p_{1}-p_{2}+\sum_{i=3}^{M} p_{i} \log _{2-2^{2-L}}\left(\frac{1}{p_{i}}\right) \\
& +\quad L+2-2 p_{1}-p_{2} \quad \frac{\mathrm{H}(\mathcal{U})+p_{1} \log _{2}\left(p_{1}\right)+p_{2} \log _{2}\left(p_{2}\right)}{\log _{2}\left(2-2^{2-L}\right)}
\end{aligned}
$$

where (41) follows from that $p_{1} \geq p_{2} \geq \cdots \geq p_{i}$ implies $p_{i} \leq \frac{1}{i}$ for $1 \leq i \leq M$.

We next study the asymptotic compression performance of UDOOCs, i.e., the situation when the source has infinitely many alphabets. Note first that with complete knowledge of the source statistics $\left\{p_{i}: i=1, \ldots, M\right\}$, the upper bound (34) in Proposition 7 can be reformulated using similar arguments as

$$
\begin{aligned}
L_{\boldsymbol{k}} \leq L+\frac{\mathrm{H}(\mathcal{U})+p_{1} \log _{2}\left(p_{1}\right)}{\log _{2}\left(g_{\boldsymbol{k}}\right)} & \\
& +\left(1-p_{1}\right)\left(1-\log _{g_{\boldsymbol{k}}}\left(T_{\boldsymbol{k}}\right)\right)
\end{aligned}
$$

where $T_{\boldsymbol{k}}$ is given by

$$
T_{\boldsymbol{k}}=\min \left\{g_{\boldsymbol{k}}^{1-n_{i}} F_{\boldsymbol{k}, n_{i}-1}: i=2, \cdots, M\right\},
$$

and $n_{i}$ is the smallest integer satisfying $F_{\boldsymbol{k}, n_{i}} \geq \frac{1}{p_{i}}$. Secondly, we can further extend the above upper bound (42) to the case of grouping $t$ source symbols (with repetition) to form a new "grouped" source for UDOOC compression. The alphabet set of the new source is therefore $\mathcal{U}^{t}$ of size $M^{t}$. Let $L_{\boldsymbol{k}, t}$ be the per-letter average codeword length of UDOOCs for the $t$ grouped source. Then, applying (42) to the $t$-grouped source yields the following upper bound on $L_{\boldsymbol{k}, t}$

$$
\begin{aligned}
L_{\boldsymbol{k}, t} \leq \frac{1}{t}\left(L+\frac{\mathrm{H}\left(\mathcal{U}^{t}\right)+q_{1} \log _{2}\left(q_{1}\right)}{\log _{2}\left(g_{\boldsymbol{k}}\right)}\right. & \\
& \left.+\left(1-q_{1}\right)\left(1-\log _{g_{\boldsymbol{k}}}\left(T_{\boldsymbol{k}, t}\right)\right)\right),
\end{aligned}
$$

where

$$
T_{\boldsymbol{k}, t}=\min \left\{g_{\boldsymbol{k}}^{1-n_{i, t}} F_{\boldsymbol{k}, n_{i, t}-1}: i=2, \cdots, M^{t}\right\},
$$

$n_{i, t}$ is the smallest integer satisfying $F_{\boldsymbol{k}, n_{i, t}} \geq \frac{1}{q_{i}}$, and $q_{i}$ is the $i$ th largest probability of the grouped source. For independent and identically distributed (i.i.d.) source, we have $\mathrm{H}\left(\mathcal{U}^{t}\right)=t \mathrm{H}(\mathcal{U})$. Moreover, assuming $M>1$ and $p_{1}<1$ for the nontrivial i.i.d. sources, we have $q_{1}=p_{1}^{t} \rightarrow 0$ as $t \rightarrow \infty$, and $T_{\boldsymbol{k}, t}$ can be shown to converge to some finite positive constant

$$
T_{\boldsymbol{k}, \infty}:=\lim _{t \rightarrow \infty} T_{\boldsymbol{k}, t}
$$

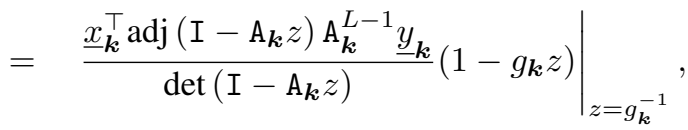

where the last step follows from the conventional expansion theory for power series and also from the fact of $g_{k}$ being the unique maximal eigenvalue of the adjacency matrix $A_{k}$ under $L>2$ (cf. Proposition 3). To elaborate, from the power series expansion, we have that $c_{\boldsymbol{k}, n}=\sum_{i} a_{i, n} \lambda_{i}^{n}+c$, where $c$ is some constant, $\left\{\lambda_{i}\right\}$ is the set of nonzero distinct eigenvalues of $\mathrm{A}_{\boldsymbol{k}}$, and $a_{i, n}$ is the coefficient associated with $\lambda_{i}$ (which could a polynomial function of $n$ if $\lambda_{i}$ has algebraic multiplicity larger than one). In particular, assuming $\lambda_{1}$ is the largest eigenvalue, we can establish that $T_{\boldsymbol{k}, \infty}=a_{1, n}=a_{1}$, where the second equality emphasizes that $a_{1, n}$ is a constant independent of $n$ since $\lambda_{1}^{-1}=g_{k}^{-1}$ is a simple zero for $h_{k}(z)$ when the digraph $G_{\boldsymbol{k}}$ is strongly connected.

By taking limits (letting $t \rightarrow \infty$ ) on both sides of (44) and by noting that $\lim _{t \rightarrow \infty} q_{1}=\lim _{t \rightarrow \infty} p_{1}^{t}=0$ and $T_{\boldsymbol{k}, \infty}$ is some finite positive constant, we summarize the asymptotic compression performance of UDOOCs in the next proposition.

Proposition 9: Given $g_{k}>1$ and a nontrivial i.i.d. source, we have

$$
\lim _{t \rightarrow \infty} L_{\boldsymbol{k}, t} \leq \frac{\mathrm{H}(\mathcal{U})}{\log _{2}\left(g_{\boldsymbol{k}}\right)} \leq \frac{\mathrm{H}(\mathcal{U})}{\log _{2}\left(2-2^{2-L}\right)}
$$

Two remarks are made based on Proposition 9. First, the larger asymptotic bound $\mathrm{H}(\mathcal{U}) / \log _{2}\left(2-2^{2-L}\right)$ in (46) immediately gives

$$
\lim _{L \rightarrow \infty} \lim _{t \rightarrow \infty} L_{\boldsymbol{k}, t}=\mathrm{H}(\mathcal{U}) .
$$

Hence, if both $t$ and $L$ are sufficiently large, the per-letter average codeword length of UDOOCs can achieve the entropy rate $\mathrm{H}(\mathcal{U})$ of the i.i.d. source. Secondly, the bound of $\mathrm{H}(\mathcal{U}) / \log _{2}\left(g_{\boldsymbol{k}}\right)$ in (46) is actually achievable by taking the all-zero UW with the source being uniformly distributed. In other words,

$$
\lim _{t \rightarrow \infty} L_{\boldsymbol{a}, t}=\frac{\mathrm{H}(\mathcal{U})}{\log _{2}\left(g_{\boldsymbol{a}}\right)}=\frac{\log _{2}(M)}{\log _{2}\left(g_{\boldsymbol{a}}\right)},
$$

where $\boldsymbol{a}=0 \ldots 0$. For better readability, we relegate the proof of (47) to Appendix D.

Tables III and IV evaluate the bounds for the English text source with letter probabilities from [36] and a true text source from Alice's Adventure in Wonderland with empirical frequencies directly obtained from the book, respectively. The source alphabet of the English text and that from Alice's Adventure in Wonderland is of size 27, where letters of upper and lower cases are regarded the same and all symbols other than the 26 English letters are treated as one. It can be observed from Table III that bound (33) is always the best among all three bounds but still has a visible gap to the resultant average codeword length $L_{\boldsymbol{k}}$. Table IV however shows that the three bounds may take turn to be on top of the other two. For example, under $\boldsymbol{k}=\boldsymbol{a},(33),(34)$ and (36) are the lowest when $(L, t)=(3,1)$, $(L, t)=(5,2)$ and $(L, t)=(6,3)$, respectively. Table IV also indicates that enlarging the value of $t$ may help improving the per-letter average codeword length as well as the bounds 
of UDOOCs. Comparison of the per-letter average codeword length of UDOOCs with the source entropy will be provided later in the simulation section.

TABLE III

UPPER BOUNDS (33), (34) AND (36) ON THE AVERAGE CODEWORD LENGTH $L_{\boldsymbol{k}}$ OF UDOOCS FOR ENGLISH TEXT SOURCE WITH LETTER PROBABILITIES FROM [36]. HERE, $\boldsymbol{a}=0 \cdots 0$ AND $\boldsymbol{b}=0 \cdots 01$.

\begin{tabular}{c|c|rrrr}
\hline $\boldsymbol{k}$ & & $L=3$ & $L=4$ & $L=5$ & $L=6$ \\
\hline \multirow{4}{*}{$\boldsymbol{a}$} & $L_{\boldsymbol{a}}$ & 6.432 & 7.411 & 8.411 & 9.411 \\
\cline { 2 - 6 } & $(33)$ & 8.330 & 9.330 & 10.330 & 11.330 \\
\cline { 2 - 6 } & $(34)$ & 9.606 & 10.496 & 11.488 & 12.484 \\
\hline \multirow{3}{*}{$\boldsymbol{b}$} & $L_{\boldsymbol{b}}$ & 5.215 & 6.185 & 7.185 & 8.185 \\
\cline { 2 - 6 } & $(33)$ & 6.553 & 7.553 & 8.553 & 9.553 \\
\cline { 2 - 6 } & $(34)$ & 10.385 & 10.206 & 10.889 & 11.769 \\
\hline- & $(36)$ & 10.831 & 10.140 & 10.652 & 11.456 \\
\hline
\end{tabular}

TABLE IV

UPPER BOUNDS (33), (34) AND (36) ON THE PER-LETTER AVERAGE CODEWORD LENGTH $L_{\boldsymbol{k}, t}$ OF UDOOCS FOR ENGLISH TEXT SOURCE FROM Alice's Adventure in Wonderland. HERE, $\boldsymbol{a}=0 \cdots 0$ AND $\boldsymbol{b}=0 \cdots 01$.

\begin{tabular}{|c|c|c|c|c|c|c|}
\hline$k$ & & & $L=3$ & $L=4$ & $L=5$ & $L=6$ \\
\hline \multirow{9}{*}{$a$} & \multirow{3}{*}{$L_{\boldsymbol{a}, t}$} & $t=1$ & 5.773 & 6.757 & 7.757 & 7.757 \\
\hline & & $t=2$ & 4.498 & 4.920 & 5.397 & 5.891 \\
\hline & & $t=3$ & 3.862 & 4.089 & 4.388 & 4.709 \\
\hline & \multirow{3}{*}{ (33) } & $t=1$ & 7.459 & 8.459 & 9.459 & 10.459 \\
\hline & & $t=2$ & 6.569 & 7.069 & 7.569 & 7.608 \\
\hline & & $t=3$ & 5.770 & 5.786 & 6.119 & 6.134 \\
\hline & \multirow{3}{*}{ (34) } & $t=1$ & 8.700 & 9.596 & 10.585 & 11.580 \\
\hline & & $t=2$ & 6.548 & 6.886 & 7.333 & 7.813 \\
\hline & & $t=3$ & 5.771 & 5.586 & 6.120 & 6.135 \\
\hline \multirow{9}{*}{$b$} & \multirow{3}{*}{$L_{\boldsymbol{b}, t}$} & $t=1$ & 4.792 & 5.774 & 6.774 & 7.774 \\
\hline & & $t=2$ & 3.791 & 4.134 & 4.598 & 5.090 \\
\hline & & $t=3$ & 3.455 & 3.532 & 3.802 & 4.115 \\
\hline & \multirow{3}{*}{ (33) } & $t=1$ & 6.716 & 6.973 & 7.973 & 8.973 \\
\hline & & $t=2$ & 6.108 & 6.147 & 6.647 & 7.147 \\
\hline & & $t=3$ & 5.452 & 5.150 & 5.483 & 5.816 \\
\hline & \multirow{3}{*}{ (34) } & $t=1$ & 9.399 & 9.366 & 10.089 & 10.984 \\
\hline & & $t=2$ & 7.356 & 6.819 & 7.040 & 7.435 \\
\hline & & $t=3$ & 5.453 & 5.150 & 5.483 & 5.817 \\
\hline \multirow{3}{*}{-} & \multirow{3}{*}{ (36) } & $t=1$ & 9.676 & 9.221 & 9.801 & 10.632 \\
\hline & & $t=2$ & 8.106 & 7.035 & 7.399 & 7.816 \\
\hline & & $t=3$ & 6.947 & 5.815 & 5.726 & 5.889 \\
\hline
\end{tabular}

\section{B. General Encoding and Decoding Mappings for UDOOCs}

In this subsection, the encoding and decoding mappings for a UDOOC with general UW are introduced.

The practice of UDOOC requires the encoding function $\phi_{\boldsymbol{k}}$ to be a bijective mapping between the subset of source letters $\mathcal{U}_{\boldsymbol{k}}(n):=\left\{u_{m}: F_{\boldsymbol{k}, n-1}<m \leq F_{\boldsymbol{k}, n}\right\}$ and the set of length$n$ codewords $\mathcal{C}_{\boldsymbol{k}}(n)$ for all $n$. Since the resulting average codeword length will be the same for any such bijective mapping from $\mathcal{U}_{\boldsymbol{k}}(n)$ to $\mathcal{C}_{\boldsymbol{k}}(n)$, we are free to devise one that facilities efficient encoding and decoding of message $u_{m}$. The bijective encoding mapping $\phi_{\boldsymbol{k}}$ that we propose is described in the following.

We define for any binary stream $\boldsymbol{d}$ of length $\leq n$,

$$
\mathcal{C}_{\boldsymbol{k}}(\boldsymbol{d}, n):=\left\{\boldsymbol{c} \in \mathcal{C}_{\boldsymbol{k}}(n): \boldsymbol{d} \text { is a prefix of } \boldsymbol{c} \text {, or } \boldsymbol{c}=\boldsymbol{d}\right\} \text {. }
$$

Obviously, $\mathcal{C}_{\boldsymbol{k}}(\boldsymbol{d}, n) \cap \mathcal{C}_{\boldsymbol{k}}(\tilde{\boldsymbol{d}}, n)=\emptyset$ for every pair of distinct $\boldsymbol{d}$ and $\tilde{\boldsymbol{d}}$ of the same length, and for any fixed $i$ with $1 \leq i \leq n$,

$$
\mathcal{C}_{\boldsymbol{k}}(n)=\bigcup_{\boldsymbol{d} \in \mathbb{F}^{i}} \mathcal{C}_{\boldsymbol{k}}(\boldsymbol{d}, n)
$$

Then, given message $u_{m} \in \mathcal{U}_{k}(n)$, i.e., the number $n$ is chosen such that $F_{\boldsymbol{k}, n-1}<m \leq F_{\boldsymbol{k}, n}$, the proposed encoding mapping $\phi_{\boldsymbol{k}}$ produces the codeword $\phi_{\boldsymbol{k}}\left(u_{m}\right)=c_{1} c_{2} \cdots c_{n}$ for source letter $u_{m}$ recursively according to the rule that for $i=1,2, \ldots, n$,

$$
c_{i}= \begin{cases}0, & \text { if } \rho_{i-1} \leq\left|\mathcal{C}_{\boldsymbol{k}}\left(c_{1} \cdots c_{i-1} 0, n\right)\right| \\ 1, & \text { if } \rho_{i-1}>\left|\mathcal{C}_{\boldsymbol{k}}\left(c_{1} \cdots c_{i-1} 0, n\right)\right|\end{cases}
$$

where the progressive metric $\rho_{i}$ is also maintained recursively as:

$$
\begin{aligned}
\rho_{i} & :=\rho_{i-1}-c_{i}\left|\mathcal{C}_{\boldsymbol{k}}\left(c_{1} \cdots c_{i-1} 0, n\right)\right| \\
& = \begin{cases}\rho_{i-1}, & \text { if } c_{i}=0 \\
\rho_{i-1}-\left|\mathcal{C}_{\boldsymbol{k}}\left(c_{1} \cdots c_{i-1} 0, n\right)\right|, & \text { if } c_{i}=1\end{cases}
\end{aligned}
$$

with an initial value $\rho_{0}=m-F_{\boldsymbol{k}, n-1}$. This encoding mapping actually assigns codewords according to their lexicographical ordering.

Example 3: Taking $\boldsymbol{k}=010$ as an example, we can see from Fig. 4 that the seven codewords of length 4, i.e., 0000, 0011, 0110, 0111, 1100, 1110 and 1111, will be respectively assigned to source letters $u_{9}, u_{10}, u_{11}, u_{12}, u_{13}, u_{14}$ and $u_{15}$. The progressive metrics $\rho_{0}, \rho_{1}, \rho_{2}, \rho_{3}$ for source letter $u_{11}$ are $3,3,1,1$, respectively, with $\left|\mathcal{C}_{\boldsymbol{k}}(0,4)\right|=4,\left|\mathcal{C}_{\boldsymbol{k}}(00,4)\right|=2$, $\left|\mathcal{C}_{\boldsymbol{k}}(010,4)\right|=0$ and $\left|\mathcal{C}_{\boldsymbol{k}}(0110,4)\right|=1$.

Note again that given $m$ (equivalently, $u_{m}$ ), $n$ can be determined via $F_{\boldsymbol{k}, n-1}<m \leq F_{\boldsymbol{k}, n}$. At the end of the $n$th recursion, we must have

$$
m=F_{\boldsymbol{k}, n-1}+\sum_{i=1}^{n} c_{i}\left|\mathcal{C}_{\boldsymbol{k}}\left(c_{1}^{i-1} 0, n\right)\right|+1 .
$$

We emphasize that (52) actually gives the corresponding computation-based decoding function $\psi_{\boldsymbol{k}}: \mathcal{C}_{\boldsymbol{k}}(n) \rightarrow \mathcal{U}_{\boldsymbol{k}}(n)$ for codewords $c$ of length $n$.

One straightforward way to implement $\phi_{\boldsymbol{k}}$ and $\psi_{\boldsymbol{k}}$ is to pre-store the value of $\left|\mathcal{C}_{\boldsymbol{k}}(\boldsymbol{d} 0, n)\right|$ for every $\boldsymbol{d}$ and $n$. By considering the huge number of all possible prefixes $\boldsymbol{d}$ for each $n$, this straightforward approach does not seem to be an attractive one.

Alternatively, we find that $\left|\mathcal{C}_{\boldsymbol{k}}(\boldsymbol{d} 0, n)\right|$ can be obtained through adjacency matrix $A_{k}$ introduced in Section II. The advantage of this alternative approach is that there is no need to pre-store or pre-construct any part of the codebook $\mathcal{C}_{\boldsymbol{k}}$, and the value of $\left|\mathcal{C}_{\boldsymbol{k}}(\boldsymbol{d} 0, n)\right|$ is computed only when it is required during the encoding or decoding processes. Moreoever, for specific UWs such as $00 \ldots 0,00 \ldots 01$, and their binary complements, we can further reduce the required computations.

In the following subsections, we will first introduce the encoding and decoding algorithms for specific UWs as they can be straightforwardly understood. Algorithms for general UWs require an additional computation of $\left|\mathcal{C}_{\boldsymbol{k}}(\boldsymbol{d} 0, n)\right|$ and will be presented in subsequent subsections.

\section{Encoding and Decoding Algorithms for $U W=11 \ldots 1$}

It has been inferred from Proposition 2 that the encoding and decoding of the UDOOC with UW $k=00 \ldots 0$ can be 
equivalently done through the encoding and decoding of the UDOOC with UW $\boldsymbol{k}=11 \ldots 1$ as one can be obtained from the other by binary complementing. Thus, we only focus on the case of $\boldsymbol{k}=11 \ldots 1$ in this subsection.

For this specific UW, we observe that a codeword $c=$ $\boldsymbol{d} 0 \boldsymbol{b} \in \mathcal{C}_{\boldsymbol{k}}(\boldsymbol{d} 0, n)$ if, and only if, $0 \boldsymbol{b} \in \mathcal{C}_{\boldsymbol{k}}(n-\ell(\boldsymbol{d}))$ is a codeword of length $n-\ell(\boldsymbol{d})$, where $\ell(\boldsymbol{d})$ is the length of prefix bitstream $\boldsymbol{d}$. We thus obtain

$$
\left|\mathcal{C}_{\boldsymbol{k}}(\boldsymbol{d} 0, n)\right|=c_{\boldsymbol{k}, n-\ell(\boldsymbol{d})} .
$$

It can be shown that the LCCDE for $c_{\boldsymbol{k}, n}$ with $\boldsymbol{k}=11 \ldots 1$ is

$$
c_{\boldsymbol{k}, n}=\sum_{i=1}^{L} c_{\boldsymbol{k}, n-i}, \text { for all } n>L,
$$

where the initial values are

$$
c_{\boldsymbol{k}, n}= \begin{cases}1, & n=0,1,2, \\ 2^{n-2}, & n=3, \ldots, L .\end{cases}
$$

Based on (53), (54) and (55), the algorithmic encoding and decoding procedures can be described below.
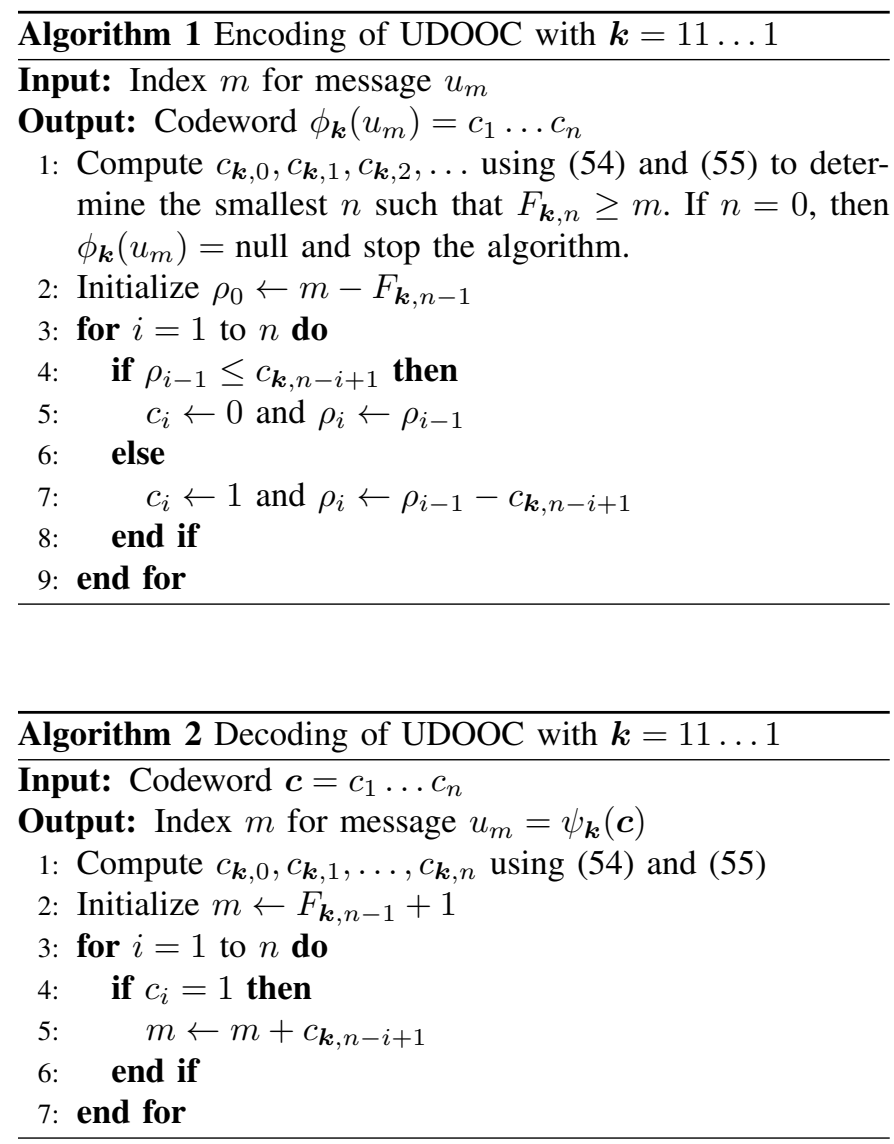

\section{Encoding and Decoding Algorithms for $11 \cdots 10$}

Again, Proposition 2 infers that the encoding and decoding of the UDOOC with UW $\boldsymbol{k}=00 \ldots 01$ can be equivalently done through the encoding and decoding of the UDOOC with UW $\boldsymbol{k}=11 \ldots 10$. We simply take $\boldsymbol{k}=11 \cdots 10$ for illustration.
It can be derived from (12) that for $k=11 \cdots 10$,

$$
c_{\boldsymbol{k}, n}=2 c_{\boldsymbol{k}, n-1}-c_{\boldsymbol{k}, n-L}
$$

with initial condition

$$
c_{\boldsymbol{k}, n}= \begin{cases}1, & n=0 \\ 2^{n}, & n=1, \ldots, L-1 \\ 2^{L}-1, & n=L .\end{cases}
$$

It remains to determine $\left|\mathcal{C}_{\boldsymbol{k}}(\boldsymbol{d} 0, n)\right|$. Observe that $\boldsymbol{d} 0 \boldsymbol{b} \in$ $\mathcal{C}_{\boldsymbol{k}}(\boldsymbol{d} 0, n)$ if, and only if, $\boldsymbol{b} \in \mathcal{C}_{\boldsymbol{k}}(n-\ell(\boldsymbol{d})-1)$; hence, $\left|\mathcal{C}_{\boldsymbol{k}}(\boldsymbol{d} 0, n)\right|=c_{\boldsymbol{k}, n-\ell(d)-1}$. We summarize the encoding and decoding algorithms of UDOOCs with $\boldsymbol{k}=11 \ldots 10$ in Algorithms 3 and 4, respectively.


\section{E. Encoding and Decoding Algorithms for General UW $\boldsymbol{k}$}

It is clear from the discussions in the previous two subsections as well as from (50) that to determine $c_{i}$ in the encoding algorithm, we only need to keep track of the most recent $\rho_{i-1}$, instead of retaining sequentially all of $\rho_{0}, \ldots, \rho_{i-2}$. We address the recursion for the update of $\rho_{i}$ in (51) only to facilitate our interpretation on the operation of the progressive metric. The same approach will be followed in the presentation of the general encoding algorithm below, where a progressive matrix $\mathrm{D}_{i}$ is used in addition to the progressive metric $\rho_{i}$.

The encoding algorithm for general UWs consists of two phases. Given the index $m$, we first identify the smallest $n$ such that $F_{\boldsymbol{k}, n} \geq m$. Note that the computation of $F_{\boldsymbol{k}, n}$ requires the knowledge of $c_{\boldsymbol{k}, n}$, which can be recursively obtained using 
the LCCDE in (18). In the second phase, as seen from the two previous subsections, we need to determine the cardinality of $\mathcal{C}_{\boldsymbol{k}}(\boldsymbol{d}, n)$ for any prefix $\boldsymbol{d}$ with $\ell(\boldsymbol{d}) \leq n$. Thus, our target in this subsection is to provide an expression for $\left|\mathcal{C}_{\boldsymbol{k}}(\boldsymbol{d}, n)\right|$ that holds for general $\boldsymbol{k}$ and $\boldsymbol{d}$.

Define $E_{\boldsymbol{k}, 0}$ and $E_{\boldsymbol{k}, 1}$ for digraph $G_{\boldsymbol{k}}=\left(V, E_{\boldsymbol{k}}\right)$ as

$$
\begin{aligned}
& E_{\boldsymbol{k}, 0}:=\left\{(\boldsymbol{i}, \boldsymbol{j}) \in E_{\boldsymbol{k}}: j_{L-1}=0\right\} \\
& E_{\boldsymbol{k}, 1}:=\left\{(\boldsymbol{i}, \boldsymbol{j}) \in E_{\boldsymbol{k}}: j_{L-1}=1\right\} .
\end{aligned}
$$

Literally speaking, $E_{\boldsymbol{k}, 0}\left(\right.$ resp. $\left.E_{\boldsymbol{k}, 1}\right)$ is the set of edges in $E_{\boldsymbol{k}}$, whose ending vertex has its last bit $j_{L-1}$ equal to 0 (resp. 1). Let $\mathrm{A}_{\boldsymbol{k}, 0}$ and $\mathrm{A}_{\boldsymbol{k}, 1}$ be the adjacency matrices respectively for digraphs $G_{\boldsymbol{k}, 0}=\left(V, E_{\boldsymbol{k}, 0}\right)$ and $G_{\boldsymbol{k}, 1}=\left(V, E_{\boldsymbol{k}, 1}\right)$. Obviously, $\mathrm{A}_{\boldsymbol{k}}=\mathrm{A}_{\boldsymbol{k}, 0}+\mathrm{A}_{\boldsymbol{k}, 1}$. Based on the two adjacency matrices, we derive

$$
\left|\mathcal{C}_{\boldsymbol{k}}(\boldsymbol{d}, n)\right|=\underline{x}_{\boldsymbol{k}}^{\top} \mathrm{D}_{i} \mathrm{~A}_{\boldsymbol{k}}^{(n+L-1)-i} \underline{y} \boldsymbol{k}
$$

where for a prefix stream $\boldsymbol{d}=d_{1} \ldots d_{i}$,

$$
\mathrm{D}_{i}:=\prod_{t=1}^{i} \mathrm{~A}_{\boldsymbol{k}, d_{t}},
$$

and $\underline{x}_{k}$ and $\underline{y}_{k}$ are the initial and ending vectors for digraph $G_{\boldsymbol{k}}$ defined in Section III-A. With (60) and (61), the general encoding and decoding algorithms are given in Algorithms 5 and 6, respectively. Verification of the two algorithms is relegated to Appendix $\mathrm{C}$ for better readability.
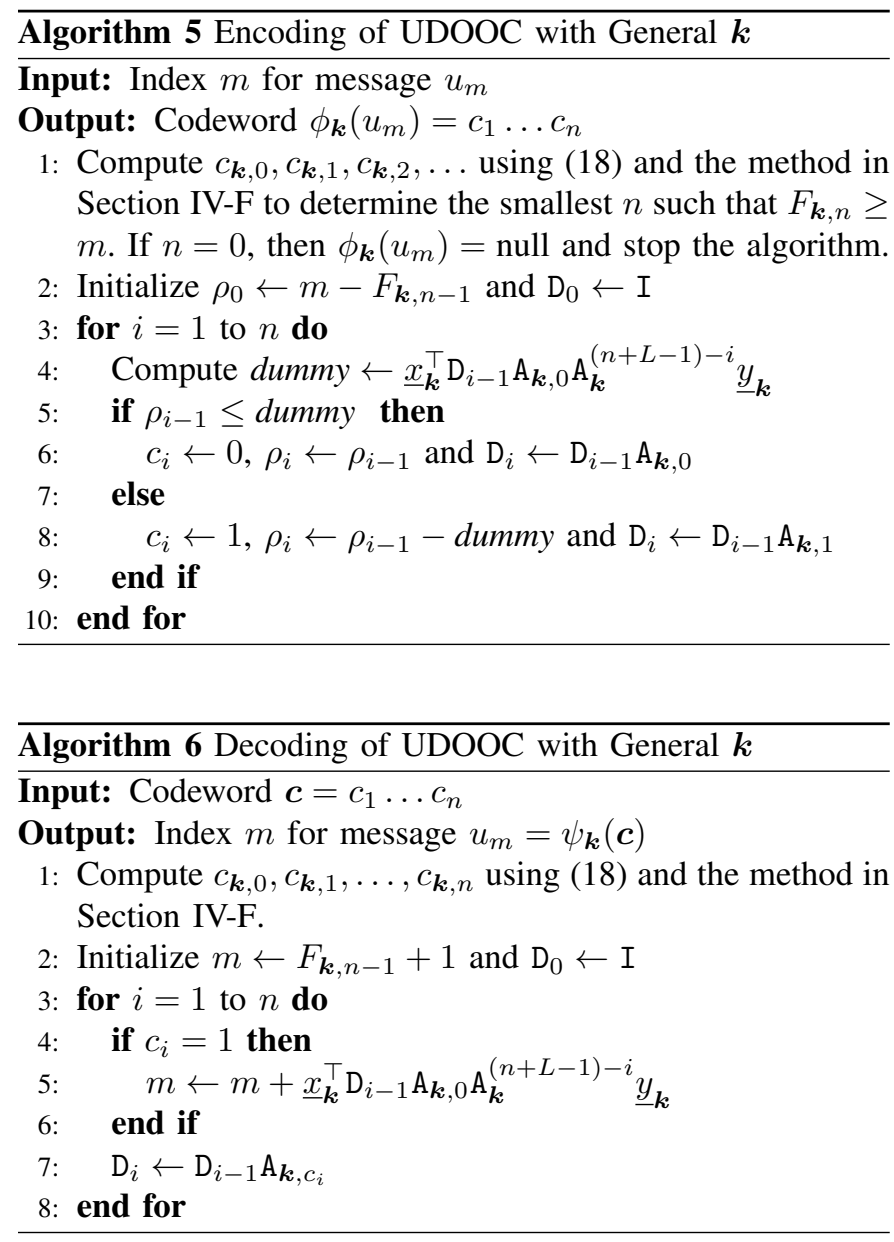

F. Exemplified Realization of the Encoding and Decoding Algorithms for General UW $\boldsymbol{k}$

The matrix expressions in (60) and (61) facilitate the presentation of Algorithms 5 and 6 for general UW; however, their implementation involves extensive computation of matrix multiplications. Since the entries in each row or column of $A_{k}$ are all 0's except for at most two 1's, the complexity of computing

$$
c_{\boldsymbol{k}, n}=\underline{x}_{\boldsymbol{k}}^{\top} \mathrm{A}_{\boldsymbol{k}}^{n+L-1} \underline{y}_{\boldsymbol{k}}
$$

and

$$
\left|\mathcal{C}_{\boldsymbol{k}}(\boldsymbol{d} 0, n)\right|=\underline{x}_{\boldsymbol{k}}^{\top} \mathrm{D}_{\ell(\boldsymbol{d})} \mathrm{A}_{\boldsymbol{k}, 0} \mathrm{~A}_{\boldsymbol{k}}^{n+L-\ell(\boldsymbol{d})-2} \underline{y}_{\boldsymbol{k}}
$$

is in fact relatively small. Furthermore, it is much easier to compute $c_{\boldsymbol{k}, n}$ than $\left|\mathcal{C}_{\boldsymbol{k}}(\boldsymbol{d} 0, n)\right|$. To see this, note from (6) that we have the following enumeration for $c_{\boldsymbol{k}, n}$

$$
\sum_{n=0}^{\infty} c_{\boldsymbol{k}, n} z^{n}=\frac{\underline{x}_{k}^{\top} \operatorname{adj}\left(\mathrm{I}-\mathrm{A}_{\boldsymbol{k}} z\right) \mathrm{A}_{\boldsymbol{k}}^{L-1} \underline{y} \boldsymbol{k}}{\operatorname{det}\left(\mathrm{I}-\mathrm{A}_{\boldsymbol{k}} z\right)} .
$$

Thus, simply evaluating the RHS of the above equation gives the values of $c_{\boldsymbol{k}, n}$ for $n=1,2, \ldots, L-1$. The remaining values of $c_{\boldsymbol{k}, n}$ for $n \geq L$ can be easily determined through the recursion formula (18).

Another way to compute the values of $c_{\boldsymbol{k}, n}$ can be easily obtained by modifying the algorithm for computing the values of $\left|\mathcal{C}_{\boldsymbol{k}}(\boldsymbol{d} 0, n)\right|$, which we now discuss. The first step to compute $\left|\mathcal{C}_{\boldsymbol{k}}(\boldsymbol{d} 0, n)\right|$ is to break up formula (63) into:

$$
\left|\mathcal{C}_{\boldsymbol{k}}(\boldsymbol{d} 0, n)\right|=\underbrace{\left(\underline{x}_{\boldsymbol{k}}^{\top} \mathrm{D}_{\ell(\boldsymbol{d})}\right)}_{\underline{u}^{\top}} \mathrm{A}_{\boldsymbol{k}, 0} \mathrm{~A}_{\boldsymbol{k}}^{n-\ell(\boldsymbol{d})-1} \underbrace{\left(\mathrm{A}_{\boldsymbol{k}}^{L-1} \underline{y}_{\boldsymbol{k}}\right)}_{\underline{w}_{k}} .
$$

We note that from the choice of $\boldsymbol{d}$ in the encoding algorithm 5, we must have $\left|\mathcal{C}_{\boldsymbol{k}}(\boldsymbol{d}, n)\right| \geq 1 .{ }^{9}$ Hence, $\underline{u}=\mathrm{D}_{\ell(\boldsymbol{d})}^{\top} \underline{x}_{\boldsymbol{k}}$ is actually a zero-one indication vector of length $2^{L-1}$ for the rightmost $(L-1)$ bits of $k_{2}^{L} \boldsymbol{d}$, i.e., all components of vector $\underline{u}=\left[\begin{array}{llll}u_{1} & u_{2} & \cdots & u_{2^{L-1}}\end{array}\right]^{\top}$ are 0 's except the $(j+1)$ th component (being 1's), where $j$ is the integer corresponding to the binary representation of the rightmost $(L-1)$ bits of $k_{2}^{L} \boldsymbol{d}$. Hence, $\underline{u}$ can be directly determined without any computation. In addition, we can pre-compute $\underline{w}_{\boldsymbol{k}}$ since it is the same for all $n$ and $\boldsymbol{d}$. Our task is therefore reduced to computing the value of

$$
\left|\mathcal{C}_{\boldsymbol{k}}(\boldsymbol{d} 0, n)\right|=\underline{u}^{\top} \mathrm{A}_{\boldsymbol{k}, 0} \mathrm{~A}_{\boldsymbol{k}}^{n-\ell(\boldsymbol{d})-1} \underline{w}_{\boldsymbol{k}} .
$$

Below, we demonstrate how to utilize a finite state machine based on the digraph $G_{\boldsymbol{k}}$ to evaluate $\left|\mathcal{C}_{\boldsymbol{k}}(\boldsymbol{d} 0, n)\right|$ without resorting to matrix operations.

Notations that are used to describe the finite state machine are addressed first. Let $\mathfrak{S}=\left\{s_{00 \cdots 0}, s_{00 \cdots 01}, \cdots, s_{11 \cdots 1}\right\}$ be the set of states indexed by all binary bit-streams of length $L-1$. We say $s_{i}=s_{i_{1} \cdots i_{L-1}}$ is a counting state if the $(i+1)$ th component of $\underline{w}_{k}$ is 1 , where $i$ is the integer corresponding to

\footnotetext{
${ }^{9}$ Given any choice of prefix $\boldsymbol{d}$, it is possible that $\left|\mathcal{C}_{\boldsymbol{k}}(\boldsymbol{d}, n)\right|=0$ if $\boldsymbol{d} \notin \mathcal{C}_{\boldsymbol{k}}$ and in this case we have $\underline{u}=\underline{0}$ in (64). However, the prefix $\boldsymbol{d}$ considered in our encoding algorithm, Algorithm 5, is always a prefix of some codeword;
} hence we have $\left|\mathcal{C}_{\boldsymbol{k}}(\boldsymbol{d}, n)\right|>0$. 
binary representation of $\boldsymbol{i}=i_{1} \ldots i_{L-1} \cdot{ }^{10}$ Denote by $\mathfrak{C}_{\boldsymbol{k}}$ the set of all counting states corresponding to $k$. Also, for each state $s_{\boldsymbol{k}} \in \mathfrak{S}$ we define

$$
\begin{aligned}
\mathcal{I}\left(s_{\boldsymbol{i}}\right) & =\left\{s_{\boldsymbol{j}}:(\boldsymbol{j}, \boldsymbol{i}) \in E_{\boldsymbol{k}}\right\} \\
\mathcal{O}\left(s_{\boldsymbol{i}}\right) & =\left\{s_{\boldsymbol{j}}:(\boldsymbol{i}, \boldsymbol{j}) \in E_{\boldsymbol{k}}\right\} \\
\mathcal{I}_{b}\left(s_{\boldsymbol{i}}\right) & =\left\{s_{\boldsymbol{j}}:(\boldsymbol{j}, \boldsymbol{i}) \in E_{\boldsymbol{k}, b}\right\} \\
\mathcal{O}_{b}\left(s_{\boldsymbol{i}}\right) & =\left\{s_{\boldsymbol{j}}:(\boldsymbol{i}, \boldsymbol{j}) \in E_{\boldsymbol{k}, b}\right\}
\end{aligned}
$$

for $b=0,1$, where the edge-sets $E_{\boldsymbol{k}, 0}$ and $E_{\boldsymbol{k}, 1}$ are defined in (58) and (59), respectively. Literally speaking, from digraph $G_{\boldsymbol{k}}, \mathcal{I}\left(s_{\boldsymbol{i}}\right)$ is the set of states that link directionally to $s_{\boldsymbol{i}}$, $\mathcal{O}\left(s_{i}\right)$ is the set of states that are linked directionally by $s_{i}$, and $\mathcal{I}_{0}\left(s_{i}\right)$ is the set of states that link to $s_{\boldsymbol{i}}$ via a so-called 0 -edge in $E_{\boldsymbol{k}, 0}$. The sets $\mathcal{I}_{1}\left(s_{\boldsymbol{i}}\right), \mathcal{O}_{0}\left(s_{\boldsymbol{i}}\right)$ and $\mathcal{O}_{1}\left(s_{\boldsymbol{i}}\right)$ all have in a similar meaning.

In our state machine, we associate each state $s_{i}$ with an integer. Without ambiguity, we use $s_{i}$ to also denote the integer associated with it. Define an operator $\Xi_{k}: \mathfrak{S} \rightarrow \mathfrak{S}$, which updates the value associated with each state according to:

$$
\Xi_{k}: s_{i} \leftarrow \sum_{s_{j} \in \mathcal{I}\left(s_{i}\right)} s_{j} \text { for all } s_{i} \in \mathfrak{S} .
$$

It should be noted that the operator $\Xi_{k}$ updates all states in $\mathfrak{S}$ in a parallel fashion. Also, if $\mathcal{I}\left(s_{i}\right)$ is an empty set, operator $\Xi_{\boldsymbol{k}}$ would set $s_{\boldsymbol{i}} \leftarrow 0$. We similarly define operators $\Xi_{\boldsymbol{k}, 0}$ and $\Xi_{\boldsymbol{k}, 1}$ respectively as

$$
\Xi_{\boldsymbol{k}, 0}: s_{\boldsymbol{i}} \leftarrow \sum_{s_{\boldsymbol{j}} \in \mathcal{I}_{0}\left(s_{\boldsymbol{i}}\right)} s_{\boldsymbol{j}} \text { and } \Xi_{\boldsymbol{k}, 1}: s_{\boldsymbol{i}} \leftarrow \sum_{s_{\boldsymbol{j}} \in \mathcal{I}_{1}\left(s_{\boldsymbol{i}}\right)} s_{\boldsymbol{j}} .
$$

An example is provided below to help clarify these notations.

Example 4: For UW $\boldsymbol{k}=000$ of length $L=3$, there are four possible states in $\mathfrak{S}=\left\{s_{00}, s_{01}, s_{10}, s_{11}\right\}$. Because $\mathrm{A}_{\boldsymbol{k}}^{L-1} \underline{y}_{\boldsymbol{k}}=\left[\begin{array}{llll}0 & 1 & 0 & 1\end{array}\right]^{\top}$, we have $\mathfrak{C}_{\boldsymbol{k}}=\left\{s_{01}, s_{11}\right\}$. Create the 0 -edges and 1-edges of the digraph in Fig. 6. Table V then shows $\mathcal{I}\left(s_{\boldsymbol{i}}\right), \mathcal{O}\left(s_{\boldsymbol{i}}\right), \mathcal{I}_{0}\left(s_{\boldsymbol{i}}\right), \mathcal{I}_{1}\left(s_{\boldsymbol{i}}\right), \mathcal{O}_{0}\left(s_{\boldsymbol{i}}\right)$ and $\mathcal{O}_{1}\left(s_{\boldsymbol{i}}\right)$ for each $s_{\boldsymbol{i}}$.

TABLE V

VARIOUS STATE SETS FOR UW $\boldsymbol{k}=000$

\begin{tabular}{|c|c|c|c|c|}
\hline$s_{\boldsymbol{i}}$ & $s_{00}$ & $s_{01}$ & $s_{10}$ & $s_{11}$ \\
\hline $\mathcal{I}\left(s_{\boldsymbol{i}}\right)$ & $\left\{s_{10}\right\}$ & $\left\{s_{00}, s_{10}\right\}$ & $\left\{s_{01}, s_{11}\right\}$ & $\left\{s_{01}, s_{11}\right\}$ \\
\hline $\mathcal{O}\left(s_{\boldsymbol{i}}\right)$ & $\left\{s_{01}\right\}$ & $\left\{s_{10}, s_{11}\right\}$ & $\left\{s_{00}, s_{01}\right\}$ & $\left\{s_{10}, s_{11}\right\}$ \\
\hline $\mathcal{I}_{0}\left(s_{\boldsymbol{i}}\right)$ & $\left\{s_{10}\right\}$ & \{\} & $\left\{s_{01}, s_{11}\right\}$ & \{\} \\
\hline $\mathcal{I}_{1}\left(s_{\boldsymbol{i}}\right)$ & \{\} & $\left\{s_{00}, s_{10}\right\}$ & \{\} & $\left\{s_{01}, s_{11}\right\}$ \\
\hline $\mathcal{O}_{0}\left(s_{\boldsymbol{i}}\right)$ & \{\} & $\left\{s_{10}\right\}$ & $\left\{s_{00}\right\}$ & $\left\{s_{10}\right\}$ \\
\hline $\mathcal{O}_{1}\left(s_{\boldsymbol{i}}\right)$ & $\left\{s_{01}\right\}$ & $\left\{s_{11}\right\}$ & $\left\{s_{01}\right\}$ & $\left\{s_{11}\right\}$ \\
\hline
\end{tabular}

According to the first row in Table $\mathrm{V}$, the operator $\Xi_{\boldsymbol{k}}$ simultaneously updates all states in $\mathfrak{S}$ according to

$$
\begin{aligned}
& s_{00} \leftarrow s_{10} \\
& \Xi_{k}: \quad \leftarrow s_{00}+s_{10} \\
& s_{10} \leftarrow s_{01}+s_{11} \\
& s_{11} \leftarrow s_{01}+s_{11}
\end{aligned}
$$

\footnotetext{
${ }^{10}$ Here we implicitly use a fact that $\underline{w}_{k}$ is a binary zero-one vector. Note that the $(i+1)$ th component of $\underline{w}_{\boldsymbol{k}}=\left[\begin{array}{llll}w_{1} & w_{2} & \cdots & w_{2^{L-1}}\end{array}\right]^{\top}=\mathrm{A}_{\boldsymbol{k}}^{L-1} \underline{y}_{\boldsymbol{k}}$ is equal to the number of distinct walks from vertex $i_{1} \cdots i_{L-1}$ to vertex $k_{1} \cdots k_{L-1}$ on digraph $G_{\boldsymbol{k}}$. This fact follows since there is at most one walk of length $(L-1)$ between the above two vertexes.
}

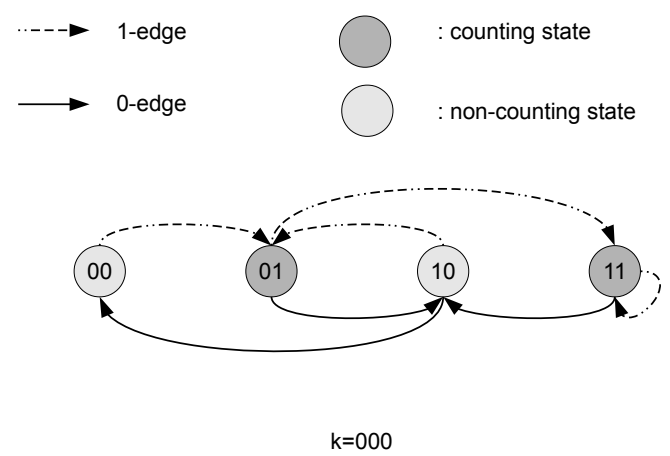

Fig. 6. Digraph $G_{000}$ for UW $\boldsymbol{k}=000$

Likewise, the operators $\Xi_{\boldsymbol{k}, 0}$ and $\Xi_{\boldsymbol{k}, 1}$ simultaneously update all states in $\mathfrak{S}$ according to

$$
\begin{array}{rlrl}
s_{00} \leftarrow s_{10} & & s_{00} \leftarrow 0 \\
\Xi_{k, 0}: & s_{01} \leftarrow 0 \\
s_{10} \leftarrow s_{01}+s_{11} & \text { and } \quad \Xi_{k, 1}: & s_{01} \leftarrow s_{00}+s_{10} \\
s_{11} \leftarrow 0 & s_{10} \leftarrow 0 \\
& & s_{11} \leftarrow s_{01}+s_{11} .
\end{array}
$$

With the above, we now demonstrate how to compute $c_{\boldsymbol{k}, i}$ and $\left|\mathcal{C}_{\boldsymbol{k}}(\boldsymbol{d} 0, n)\right|$ using the finite state machine. Note $c_{\boldsymbol{k}, n}=$ $\underline{x}_{k}^{\top} \mathrm{A}_{\boldsymbol{k}}^{n} \underline{w}_{\boldsymbol{k}}$; hence to compute $c_{\boldsymbol{k}, n}$, the states are initialized such that $s_{k_{2} \ldots k_{L}}=1$ and $s_{i}=0$ for all remaining $\boldsymbol{i} \neq k_{2}^{L}$. Note that these initial values correspond exactly to the component values of vector $\underline{x}_{\boldsymbol{k}}$. Next we apply $n$ times the operator $\Xi_{\boldsymbol{k}}$ to update the states in $\mathfrak{S}$. It can be seen that the resulting values of the states correspond exactly to the contents of the row vector $\underline{x}_{\boldsymbol{k}}^{\top} \mathrm{A}_{\boldsymbol{k}}^{n}$. Thus, the value of $c_{\boldsymbol{k}, n}$ can be obtained by summing the values of the counting states. Again, we remark that we only need the finite state machine for computing the values of $c_{\boldsymbol{k}, n}$ for $n=1,2, \ldots, L-1$, as the values of $c_{\boldsymbol{k}, n}$ for $n \geq L$ can be easily determined by the recursion formula (18).

On the other hand, to compute

$$
\left|\mathcal{C}_{\boldsymbol{k}}(\boldsymbol{d} 0, n)\right|=\underline{u}^{\top} \mathrm{A}_{\boldsymbol{k}, 0} \mathrm{~A}_{\boldsymbol{k}}^{n-\ell(\boldsymbol{d})-1} \underline{w}_{\boldsymbol{k}}
$$

for a given prefix $\boldsymbol{d}$, we initialize the values associated with all states to be zero except $s_{u_{m-L+2} \cdots u_{m}}=1$, where $u_{m-L+2} \cdots u_{m}$ is the rightmost $(L-1)$ elements in $\boldsymbol{u}=$ $u_{1} \ldots u_{m}=k_{2}^{L} \boldsymbol{d}$. Apply the operator $\Xi_{\boldsymbol{k}, 0}$ to all states in $\mathfrak{S}$ once, followed by updating all the states $(n-\ell(\boldsymbol{d})-1)$ times via operator $\Xi_{\boldsymbol{k}}$. Then, the sum of the values of all counting states equals $\left|\mathcal{C}_{\boldsymbol{k}}(\boldsymbol{d} 0, n)\right|$.

\section{PRACTICE AND PERFORMANCE OF UDOOCS}

In Fig. 7, we compare the numbers of length- $n$ codewords for all UWs of lengths $L=2,3,4$ and 5. These numbers are plotted in logarithmic scale and are normalized against the number of length- $n$ codewords for the all-zero 
UW $k=0 \ldots 00$ to facilitate their comparison. By the equivalence relation defined in Definition 2, only one UW in each equivalence class needs to be illustrated. We have the following observations.

1) The logarithmic ratio $\log _{2}\left(c_{\boldsymbol{k}, n} / c_{\boldsymbol{a}, n}\right)$, where $\boldsymbol{a}=$ $0 \ldots 00$, exhibits some transient fluctuation for $n \leq L$ but becomes a steady straight line of negative slope after $n>L$. This hints that $c_{\boldsymbol{k}, n}$ has a steady exponential growth when $n$ is beyond $L$.

2) The number $c_{\boldsymbol{b}, n}$, where $\boldsymbol{b}=00 \ldots 01$, is always the largest among all $c_{\boldsymbol{k}, n}$ when $n$ is small. However, this number has an apparent trend to be overtaken by those of other UWs as $n$ grows and will be eventually smaller than the number of length- $n$ codewords for the all-zero UW. This result matches the statement of Theorem 2 .

3) As a contrary, the number $c_{\boldsymbol{a}, n}$ for the all-zero UW $\boldsymbol{a}=0 \ldots 00$ is the smallest among all $c_{\boldsymbol{k}, n}$ for UWs of the same length when $n$ is small. Although Theorem 2 indicates that this number will eventually be the largest, Fig. 7 shows that such would happen only when $n$ is very large.

4) As a result of the two previous observations, UW $\boldsymbol{b}=00 \ldots 01$ perhaps remains a better choice in the compression of sources with practical number of source letters even though it is asymptotically the worst. We will confirm this inference by the later practice of UDOOCs on a real text source from the book Alice's Adventure in Wonderland.

We next investigate the compression rates of UDOOCs and compare them with those of the Huffman and LempelZiv (specifically, LZ77 and LZ78) codes. In this experiment, the standard Huffman code in the communication toolbox of Matlab is used instead of the adaptive Huffman code. The LZ77 executable is obtained from the basic compression library in [34], while the LZ78 is self-implemented using $\mathrm{C}++$ programing language. As a convention, the data is binary ASCII encoded before it is fed into the two Lempel-Ziv compression algorithms. The sliding window for the LZ77 is set as 10,000 bits, and the tree-structured LZ78 is implemented without any windowing.

Three different English text sources are used, in which the uppercase and lowercase of each English letter are treated as the same symbol. The first English text source is distributed uniformly over the 26 symbols. The second English text source is assumed independent and identically distributed (i.i.d.) with marginal statistics from [36]. The third one is a realistic English text source from Alice's Adventure in Wonderland, in which any symbols other than the 26 English alphabets are regarded as a "space." In addition, the effect of grouping $t$ symbols as a grouped source for compression is studied, which will be termed $t$-grouper in remarks below. The results are summarized in Tables VI and VII, in which the average codeword length of UDOOCs has already taken into account the length of UWs. We remark on the experimental results as follows.

1) First of all, it can be observed from Table VI that the length-2 UW $\boldsymbol{k}=01$ gives a good per-letter average codeword length only when $t=1$. When the size of source alphabet increases by grouping $t=2$ or $t=3$ letters as one symbol for UDOOC compression, the per-letter average codeword length dramatically grows. Note that $\boldsymbol{k}=01$ is the only UW, whose number of length- $n$ codewords has a linear growth with respect to $n$, i.e., we have $c_{01, n}=n+1$. Since the size of source alphabets increases exponentially in $t$ when $t$ grouper is employed, the resulting per-letter average codeword length also increases exponentially as $t$ grows. Therefore, when UW $=01, t$-grouper will result in an extremely poor performance for moderately large $t$.

2) By independently generating $10^{6}$ letters according to the statistics in [36] for compression, we record the per-letter average codeword in the second row of Table VI. As expected, the Huffman coding scheme gives the smallest per-letter average codeword length of 4.253 bits per letter, when 3 -grouper is used. The gap of perletter average codeword lengths between the 3-grouper Huffman and the 3-grouper UDOOC however can be made as small as $4.795-4.253=0.542$ bits per source letter if $\mathrm{UW}=0001$. This is in contrast to the gap of 1.007 bits when uniform independent English text source is the one to be compressed (cf. the first row in Table VI). We would like to point out that the error propagation of UDOOCs is limited firmly by at most two codewords, while that of the Huffman code may be statistically beyond this range. In comparison with the LZ77 and LZ78, the UDOOC clearly performs better in compression rate for usual independent English text source.

3) When the compression of a source with memory such as the book titled Alice's Adventures in Wonderland [35] is concerned, the third row in Table VI shows that the gap of per-letter average codeword lengths between the optimal 3-grouper Huffman and the 3-grouper UDOOC with $\mathrm{UW}=0001$ is narrowed down to 0.305 bits per letter. The 3-grouper UDOOC with the all-zero UW also performs well for this source. Note that part of the per-letter average codeword length of UDOOCs is contributed by the UW, i.e., $L / t$; hence, in a sense, a larger $t$ and a smaller $L$ are favored (except for $L=2$ ). As can be seen from Table VI, the best compression performance is given by $t=3, L=4$, and $\mathrm{UW}=0001$.

4) For the third English text source, the LZ77 performs better than all of the 1-grouper UDOOC compression schemes but one. We then compare the running time of both algorithms. We reduce the window size of LZ77 so that it has a similar running time to the 1grouper UDOOC scheme. The compression performance of LZ77 degrades down to 5.234 bits per letter, which is larger than that of the 1-grouper UDOOC. Note that we only compare their running time in encoding in Table VII as the decoding efficiency of UDOOCs is seemingly better than that of the LZ77. Considering also the low memory consumption of UDOOCs when a specific UW is pre-given in addition to its simplicity in implementation, the UDOOC can be regarded as a cost- 


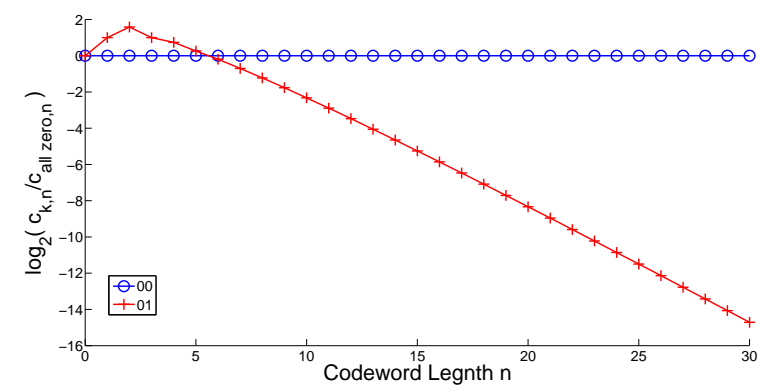

(a) $L=2$



(c) $L=4$

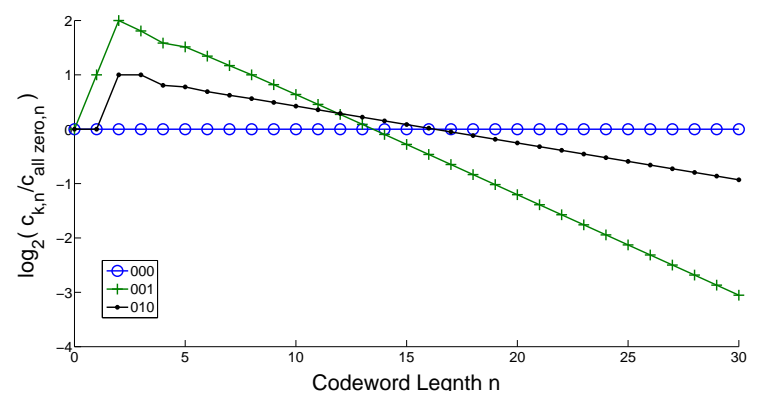

(b) $L=3$

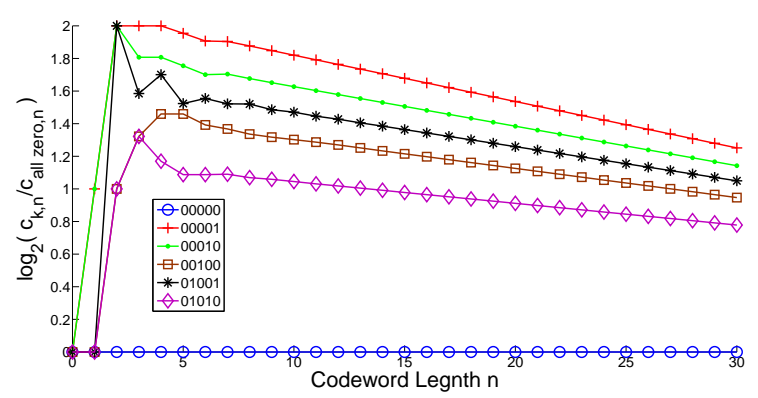

(d) $L=5$

Fig. 7. Normalized numbers of length- $n$ codewords for UWs of lengths $L=2,3,4,5$

effective compression scheme for practical applications.

\section{TABLE VII}

AVERAGE CODEWORD LENGTHS IN BITS PER SOURCE SYMBOL AND RUNNING TIME IN SECONDS FOR THE UDOOC ENCODING AND THE LZ77 ENCODING ON Alice's Adventures in Wonderland. THE PROGRAMS ARE IMPLEMENTED USING C++, AND ARE EXECUTED IN A MICROSOFT WINDOWS-BASED DESKTOP WITH INTEL-CORE7 2.4G CUP AND 8G MEMORY.

\section{APPENDIX A \\ PROOF OF THEOREM 1}

In this section, we will prove (11), the enumeration of $s_{\boldsymbol{k}, n}$ in Theorem 1. Our proof technique is similar to that in [23].

Let $\mathbb{F}^{\infty}:=\bigcup_{n \geq 0} \mathbb{F}^{n}$ be the set of all binary sequences. For a word $\boldsymbol{w}=w_{1} \ldots w_{n} \in \mathbb{F}^{\infty}$ of length $n$, let $\mathcal{F}_{\boldsymbol{k}}(\boldsymbol{w})$ be the set of index pairs indicating the places that $\boldsymbol{w}$ contains $\boldsymbol{k}$ as a subword, i.e.,

\begin{tabular}{c|c|c|c}
\hline \hline \multicolumn{2}{|c|}{ Type } & Average Codewrod length & Running Time \\
\hline \multirow{2}{*}{ UDOOC } & UW $\boldsymbol{k}=00$ & 4.887 & $0.0162 \mathrm{sec}$ \\
\cline { 2 - 4 } & UW $\boldsymbol{k}=01$ & 4.068 & $0.0158 \mathrm{sec}$ \\
\hline \multirow{2}{*}{ LZ77 } & Window Size $=10^{4}$ bits & 4.661 & $0.0328 \mathrm{sec}$ \\
\cline { 2 - 4 } & Window Size $=3000$ bits & 5.234 & $0.01607 \mathrm{sec}$ \\
\hline \hline
\end{tabular}

\section{CONCLUSION}

In this paper, we have provided a general construction of UDOOCs with arbitrary UW. Combinatorial properties of UDOOCs are subsequently investigated. Based on our studies, the appropriate UW for the UDOOC compression of a given source can be chosen. Various encoding and decoding algorithms for general UDOOCs, as well as their efficient counterparts for specific UWs like $k=00 \ldots 0$, $00 \ldots 01$, are also provided. Performances of UDOOCs are then compared with the Huffman and Lempel-Ziv codes. Our experimental results show that the UDOOC can be a good practical candidate for lossless data compression when a costefficient solution is desired.

$$
\mathcal{F}_{\boldsymbol{k}}(\boldsymbol{w})=\left\{(i, j): \boldsymbol{k}=w_{i}^{j}\right\}
$$

Further denote by $\ell(\boldsymbol{w})$ the length of word $\boldsymbol{w}$. Then

$$
\begin{aligned}
f(z) & =\sum_{n \geq 0} s_{\boldsymbol{k}, n} z^{n} \\
& \stackrel{(\mathrm{i})}{=} \sum_{\boldsymbol{w} \in \mathbb{F}^{\infty}} z^{\ell(\boldsymbol{w})} 0^{\left|\mathcal{F}_{\boldsymbol{k}}(\boldsymbol{w})\right|} \\
& =\sum_{\boldsymbol{w} \in \mathbb{F}^{\infty}} z^{\ell(\boldsymbol{w})} \prod_{a \in \mathcal{F}_{\boldsymbol{k}}(\boldsymbol{w})}(1+(-1)) \\
& \stackrel{(\mathrm{ii})}{=} \sum_{\boldsymbol{w} \in \mathbb{F}^{\infty}} z^{\ell(\boldsymbol{w})} \sum_{A \subseteq \mathcal{F}_{\boldsymbol{k}}(\boldsymbol{w})}(-1)^{|A|}
\end{aligned}
$$

where in (i) we have adopted the convention of $0^{0}=1$, and (ii) follows from the inclusion-exclusion principle. In light of (65), we will regard the pair $(\boldsymbol{w}, A)$ with $A \subseteq \mathcal{F}_{\boldsymbol{k}}(\boldsymbol{w})$ as a marked word. The set of all marked words is thus defined as

$$
\mathcal{M}_{\boldsymbol{k}}:=\left\{(\boldsymbol{w}, A): \boldsymbol{w} \in \mathbb{F}^{\infty} \text { and } A \subseteq \mathcal{F}_{\boldsymbol{k}}(\boldsymbol{w})\right\}
$$

Define the following weight function for elements in $\mathcal{M}_{\boldsymbol{k}}$

$$
\pi(\boldsymbol{w}, A):=z^{\ell(\boldsymbol{w})}(-1)^{|A|}
$$


TABLE VI

AVERAGE CODEWORD LENGTHS IN BITS PER SOURCE SYMBOL FOR THE COMPRESSION OF THREE DIFFERENT SOURCES. THE BEST ONE AMONG 1-GROUPER, 2-GROUPER AND 3-GROUPER OF THE SAME COMPRESSION SCHEME IS BOLDFACED.

\begin{tabular}{|c|c|c|c|c|c|c|c|c|c|c|c|c|c|c|c|}
\hline Type & \multicolumn{3}{|c|}{ Entropy } & \multirow[t]{2}{*}{ LZ77 } & \multirow[t]{2}{*}{ LZ78 } & \multicolumn{3}{|c|}{ Huffman } & & \multicolumn{3}{|c|}{$\mathbf{U W}=00 \cdots 0$} & \multicolumn{3}{|c|}{$\mathbf{U W}=00 \cdots 01$} \\
\hline \multirow[b]{2}{*}{$\begin{array}{c}\text { Independent } \\
\text { English Letter with } \\
\text { Uniform distribution }\end{array}$} & $t=1$ & $t=2$ & $t=3$ & & & $t=1$ & $\bar{t} t=2$ & $t=3$ & & $t=1$ & $t=2$ & $t=3$ & $t=1$ & $t=2$ & $t=3$ \\
\hline & 4.700 & 4.700 & 4.700 & 7.992 & 7.178 & 4.768 & 4.738 & 4.702 & $\begin{array}{l}L=2 \\
L=4 \\
L=6\end{array}$ & $\begin{array}{l}6.961 \\
8.576 \\
10.58\end{array}$ & $\begin{array}{l}6.820 \\
6.831 \\
7.748\end{array}$ & $\begin{array}{l}6.790 \\
6.213 \\
6.746\end{array}$ & $\begin{array}{l}\mathbf{5 . 8 4 6} \\
7.000 \\
9.000\end{array}$ & $\begin{array}{l}12.76 \\
5.899 \\
6.768\end{array}$ & $\begin{array}{l}41.99 \\
\mathbf{5 . 7 0 9} \\
\mathbf{6 . 1 0 4}\end{array}$ \\
\hline \multirow[b]{2}{*}{$\begin{array}{c}\text { Independent } \\
\text { English Letter with } \\
\text { Usual distribution }\end{array}$} & $t=1$ & $t=2$ & $t=3$ & & & $t=1$ & $t=2$ & $t=3$ & & $t=1$ & $t=2$ & $t=3$ & $t=1$ & $t=2$ & $t=3$ \\
\hline & 4.246 & 4.246 & 4.246 & 7.925 & 6.626 & 4.274 & 4.261 & 4.253 & $\begin{array}{l}L=2 \\
L=4 \\
L=6\end{array}$ & $\begin{array}{l}5.591 \\
7.411 \\
9.411 \\
\end{array}$ & $\begin{array}{l}\mathbf{5 . 5 5 0} \\
5.872 \\
6.818 \\
\end{array}$ & $\begin{array}{l}5.637 \\
\mathbf{5 . 3 5 1} \\
\mathbf{5 . 9 2 4} \\
\end{array}$ & $\begin{array}{l}4.557 \\
6.185 \\
8.185 \\
\end{array}$ & $\begin{array}{l}7.771 \\
4.970 \\
5.882 \\
\end{array}$ & $\begin{array}{r}20.907 \\
\mathbf{4 . 7 9 5} \\
\mathbf{5 . 2 7 4} \\
\end{array}$ \\
\hline \multirow[b]{2}{*}{$\begin{array}{c}\text { Alice's } \\
\text { Adventures } \\
\text { in Wonderland }\end{array}$} & $\bar{t} t=1$ & $\bar{t} t=2$ & $\bar{t} t=3$ & & & $\bar{t} t=1$ & $\bar{t} t=2$ & $\bar{t} t=3$ & & $\bar{t} t=1$ & $\bar{t} t=2$ & $\bar{t} t=3$ & $\bar{t} t=1$ & $\bar{t} t=2$ & $\bar{t} t=3$ \\
\hline & 3.914 & 3.570 & 3.215 & 4.661 & 6.028 & 3.940 & 3.585 & 3.226 & $\begin{array}{l}L=2 \\
L=4 \\
L=6\end{array}$ & $\begin{array}{l}4.887 \\
6.757 \\
8.757 \\
\end{array}$ & $\begin{array}{l}4.340 \\
4.920 \\
5.890\end{array}$ & $\begin{array}{l}3.958 \\
4.089 \\
4.709\end{array}$ & $\begin{array}{l}\mathbf{4 . 0 6 8} \\
5.774 \\
7.774 \\
\end{array}$ & $\begin{array}{l}4.975 \\
4.133 \\
5.089\end{array}$ & $\begin{array}{l}7.573 \\
\mathbf{3 . 5 3 1} \\
\mathbf{4 . 1 1 5}\end{array}$ \\
\hline
\end{tabular}

then (65) can be rewritten as

$$
f(z)=\sum_{(\boldsymbol{w}, A) \in \mathcal{M}_{\boldsymbol{k}}} \pi(\boldsymbol{w}, A)
$$

To determine $f(z)$, below we introduce the concept of a cluster.

Definition 6 (Cluster): We say the marked word $(\boldsymbol{w}, A)$ is a cluster if, and only if,

$$
\bigcup_{\left(i_{t}, j_{t}\right) \in A}\left[i_{t}, j_{t}\right]=[1, \ell(\boldsymbol{w})]
$$

where by $[a, b]$ we mean the closed interval $\{x \in \mathbb{R}: a \leq x \leq$ $b\}$ on the real line. The set of all clusters is thus

$$
\mathcal{T}_{\boldsymbol{k}}=\left\{(\boldsymbol{w}, A) \in \mathcal{M}_{\boldsymbol{k}}:(\boldsymbol{w}, A) \text { is a cluster }\right\} .
$$

Definition 7 (Concatenation of sets of marked words): For any two sets of marked words $\mathcal{A}_{k}$ and $\mathcal{B}_{k}$, we define the concatenation of $\mathcal{A}_{k}$ and $\mathcal{B}_{k}$ as

$$
\mathcal{A}_{\boldsymbol{k}} \vee \mathcal{B}_{\boldsymbol{k}}:=\left\{\left(\boldsymbol{a} \boldsymbol{b}, A \cup \mathfrak{J}(B, \ell(\boldsymbol{a})):(\boldsymbol{a}, A) \in \mathcal{A}_{\boldsymbol{k}},(\boldsymbol{b}, B) \in \mathcal{B}_{\boldsymbol{k}}\right\}\right.
$$

where by $\boldsymbol{a} \boldsymbol{b}$ we meant the usual concatenation of strings $\boldsymbol{a}$ and $\boldsymbol{b}$, and the function $\mathfrak{J}(B, \ell(\boldsymbol{a}))$ is

$$
\mathfrak{J}(B, \ell(\boldsymbol{a})):=\left\{\left(i_{t}+\ell(\boldsymbol{a}), j_{t}+\ell(\boldsymbol{a})\right):\left(i_{t}, j_{t}\right) \in B\right\} .
$$

Having defined the concatenation operation $\vee$ for sets of marked words, we next claim the following decomposition for the set $\mathcal{M}_{k}$

$$
\mathcal{M}_{k}=\{(\text { null }, \emptyset)\} \cup\left(\mathcal{M}_{k} \vee \mathcal{F}\right) \cup\left(\mathcal{M}_{k} \vee \mathcal{T}_{k}\right),
$$

where $\mathcal{F}:=\{(b, \emptyset): b \in \mathbb{F}\}$.

To show (68), for any $(\boldsymbol{w}, A) \in \mathcal{M}_{\boldsymbol{k}}$ we distinguish the following three disjoint cases:

1) If $\ell(\boldsymbol{w})=0$, it is obvious that $\boldsymbol{w}$ is a null word and $A=\emptyset$ from the definition of $\mathcal{F}_{\boldsymbol{k}}(\boldsymbol{w})$.

2) For $\ell(\boldsymbol{w}) \geq 1$, appending an arbitrary binary word to $\boldsymbol{w}$ results in another marked word $(\boldsymbol{w} b, A)$, which cannot be a cluster since

$$
\bigcup_{\left(i_{t}, j_{t}\right) \in A}\left[i_{t}, j_{t}\right] \subset[1, \ell(\boldsymbol{w})+1] .
$$

Conversely, take any marked word $(\boldsymbol{w}, A)$ from $\mathcal{M}_{\boldsymbol{k}}$ with $\ell(\boldsymbol{w})=n$. If $j_{t}<\ell(\boldsymbol{w})=n$ for all $\left(i_{t}, j_{t}\right) \in A$, then we can delete the rightmost bit from $\boldsymbol{w}$, and the resulting pair $\left(w_{1}^{n-1}, A\right)$ is still a marked word. Summarizing the above gives the following equalities between two sets of marked words

$$
\begin{aligned}
& \left.\left\{(\boldsymbol{w}, A) \in \mathcal{M}_{\boldsymbol{k}}: j_{t}<\ell(\boldsymbol{w}) \text { for all }\left(i_{t}, j_{t}\right) \in A\right)\right\} \\
& \quad=\left\{(\boldsymbol{w} b, A):(\boldsymbol{w}, A) \in \mathcal{M}_{\boldsymbol{k}}, b \in \mathbb{F}\right\} \\
& \quad=\mathcal{M}_{\boldsymbol{k}} \vee \mathcal{F},
\end{aligned}
$$

where the last equality follows from the definition of concatenation operation $V$.

3) The last case concerns the situation when $(\boldsymbol{w}, A)$ satisfies $\ell(\boldsymbol{w})=n \geq 1, A=\left\{\left(i_{1}, j_{1}\right), \ldots,\left(i_{m}, j_{m}\right)\right\}$ and $i_{1}<\cdots<i_{m}<j_{m}=n$. In other words, this is the case when $\max \left\{j_{t}:\left(i_{t}, j_{t}\right) \in A\right\}=\ell(\boldsymbol{w})$, which is disjoint from the second case. For this, let $u$ be the smallest index such that $\left[i_{u+t}, j_{u+t}\right] \cap\left[i_{u+t+1}, j_{u+t+1}\right] \neq \emptyset$ for all $t=0,1, \ldots, m-u+1$. Then obviously we have the following de-concatenation of $(\boldsymbol{w}, A)$

$$
\begin{aligned}
& (\boldsymbol{w}, A)=\left(w_{1}^{i_{u}-1},\left\{\left(i_{t}, j_{t}\right): t=1, \ldots, u-1\right\}\right) \\
& \quad \vee\left(w_{i_{u}}^{n},\left\{\left(i_{t}-i_{u}+1, j_{t}-i_{u}+1\right): t=u, \ldots, m\right\}\right) .
\end{aligned}
$$

Clearly, the first marked word $\left(w_{1}^{i_{u}-1},\left\{\left(i_{t}, j_{t}\right): t=\right.\right.$ $1, \ldots, u-1\}) \in \mathcal{M}_{\boldsymbol{k}}$. The second marked word $\left(w_{i_{u}}^{n},\left\{\left(i_{t}-i_{u}+1, j_{t}-i_{u}+1\right): t=u, \ldots, m\right\}\right)$ is a cluster since

$$
\bigcup_{t=u}^{m}\left[i_{t}-i_{u}+1, j_{t}-i_{u}+1\right]=\left[1, n-i_{u}+1\right]
$$

by the choice of $u$. Hence we arrive at the following equality between two sets of marked words

$$
\begin{aligned}
& \left\{(\boldsymbol{w}, A) \in \mathcal{M}_{\boldsymbol{k}}: \max \left\{j_{t}:\left(i_{t}, j_{t}\right) \in A\right\}=\ell(\boldsymbol{w})\right\} \\
& \quad=\mathcal{M}_{\boldsymbol{k}} \vee \mathcal{T}_{\boldsymbol{k}} .
\end{aligned}
$$

Combining the case of null word and equations (69) and (70) proves the desired claim of (68).

Using the decomposition in (68), we can rewrite (67) in terms of the three sets, i.e., the set for null word, $\mathcal{M}_{\boldsymbol{k}} \vee \mathcal{F}$, 
and $\mathcal{M}_{\boldsymbol{k}} \vee \mathcal{T}_{\boldsymbol{k}}$. In particular, we have

$$
\begin{aligned}
& \sum_{(\boldsymbol{w}, W) \in \mathcal{M}_{\boldsymbol{k}} \vee \mathcal{T}_{\boldsymbol{k}}} \pi(\boldsymbol{w}, W) \\
= & \sum_{(\boldsymbol{a}, A) \in \mathcal{M}_{\boldsymbol{k}}} \sum_{(\boldsymbol{b}, B) \in \mathcal{T}_{\boldsymbol{k}}} z^{\ell(\boldsymbol{a} \boldsymbol{b})}(-1)^{|A \cup \mathfrak{J}(B, \ell(\boldsymbol{a}))|} \\
= & \sum_{(\boldsymbol{a}, A) \in \mathcal{M}_{\boldsymbol{k}}} \sum_{(\boldsymbol{b}, B) \in \mathcal{T}_{\boldsymbol{k}}} z^{\ell(\boldsymbol{a})+\ell(\boldsymbol{b})}(-1)^{|A|+|B|} \\
= & \left(\sum_{(\boldsymbol{a}, A) \in \mathcal{M}_{\boldsymbol{k}}} \pi(\boldsymbol{a}, A)\right)\left(\sum_{(\boldsymbol{b}, B) \in \mathcal{T}_{\boldsymbol{k}}} \pi(\boldsymbol{b}, B)\right) .
\end{aligned}
$$

Similarly, one can show that

$$
\sum_{(\boldsymbol{w}, A) \in \mathcal{M}_{\boldsymbol{k}} \vee \mathcal{F}_{2}} \pi(\boldsymbol{w}, A)=2 z \sum_{(\boldsymbol{w}, A) \in \mathcal{M}_{\boldsymbol{k}}} \pi(\boldsymbol{w}, A) .
$$

Substituting (71) and (72) into (67) gives

$$
f(z)=\sum_{(\boldsymbol{w}, A) \in \mathcal{M}_{\boldsymbol{k}}} \pi(\boldsymbol{w}, A)=1+2 z f(z)+f(z) T(z),
$$

or equivalently,

$$
f(z)=\frac{1}{1-2 z-T(z)},
$$

where $T(z)$ is the weight enumerator of elements in $\mathcal{T}_{\boldsymbol{k}}$ given by

$$
T(z):=\sum_{(\boldsymbol{b}, B) \in \mathcal{T}_{\boldsymbol{k}}} \pi(\boldsymbol{b}, B) .
$$

Determining $T(z)$ is now relatively easy. Recall that the overlap function $r_{\boldsymbol{k}}(i)=\mathbf{1}\left(k_{1}^{L-i}=k_{i+1}^{L}\right)$, where $\mathbf{1}(\cdot)$ is the usual indicator function, shows exactly whether the length- $(L-i)$ prefix of $\boldsymbol{k}$ is also a suffix of $\boldsymbol{k}$. Let $\mathcal{R}_{\boldsymbol{k}}=$ $\left\{i: 1 \leq i \leq L-1, r_{\boldsymbol{k}}(i)=1\right\}$. For any cluster $(\boldsymbol{b}, B) \in \mathcal{T}_{\boldsymbol{k}}$ with $\boldsymbol{b}=b_{1} \ldots b_{n}$, we must have $b_{n-L+1}^{n}=\boldsymbol{k}$ by Definition 6. So for any $i \in \mathcal{R}_{\boldsymbol{k}}$, i.e., $r_{\boldsymbol{k}}(i)=1$, we have $b_{n-L+i+1}^{n}=$ $k_{i+1}^{L}=k_{1}^{L-i}$. Hence the pair

$$
\left(\boldsymbol{b} k_{L-i+1} \ldots k_{L}, B \cup\{(n+i-L+1, n+i)\}\right)
$$

is a cluster in $\mathcal{T}_{\boldsymbol{k}}$. It implies that for $i \in \mathcal{R}_{\boldsymbol{k}}$, the set

$$
\mathcal{T}_{\boldsymbol{k}, i}:=\left\{\begin{array}{r}
\left(\boldsymbol{b} k_{L-i+1}^{L}, B \cup\{(n+i-L+1, n+i)\}\right): \\
(\boldsymbol{b}, B) \in \mathcal{T}_{\boldsymbol{k}}, n=\ell(\boldsymbol{b})
\end{array}\right\}
$$

is a subset of $\mathcal{T}_{\boldsymbol{k}}$.

On the other hand, take any $(\boldsymbol{b}, B) \in \mathcal{T}_{\boldsymbol{k}}$ with $\ell(\boldsymbol{b})=n$ and $B=\left\{\left(i_{t}, j_{t}\right): t=1, \ldots, m\right\}$, where $1=i_{1}<i_{2}<$ $\cdots<i_{m}<j_{m}=n$ and $i_{m}=n-L+1$. If $m=1$, then $\boldsymbol{b}=\boldsymbol{k}$ and $B=\{(1, L)\}$. Hence we consider the case when $m>1$. As $(\boldsymbol{b}, B)$ is a cluster, $\left[i_{m-1}, j_{m-1}\right] \cap\left[i_{m}, j_{m}\right] \neq \emptyset$ and $b_{i_{m-1}}^{j_{m-1}}=b_{i_{m}}^{j_{m}}=\boldsymbol{k}$. Therefore, we must have $b_{i_{m}}^{j_{m-1}}=k_{1}^{v}=$ $k_{L-v+1}^{L}$, where $v=j_{m-1}-i_{m}+1$. Thus, $r_{\boldsymbol{k}}(L-v)=1$ and $(\boldsymbol{b}, B) \in \mathcal{T}_{\boldsymbol{k}, L-v}$. The above discussion then gives the following decomposition for $\mathcal{T}_{k}$

$$
\mathcal{T}_{\boldsymbol{k}}=\{(\boldsymbol{k},\{(1, L)\})\} \cup\left(\bigcup_{i \in \mathcal{R}_{\boldsymbol{k}}} \mathcal{T}_{\boldsymbol{k}, i}\right) .
$$

For enumerating the weights of elements in $\mathcal{T}_{\boldsymbol{k}}$, we further claim that $\mathcal{T}_{\boldsymbol{k}, i} \cap \mathcal{T}_{\boldsymbol{k}, j}=\emptyset$ for all $i \neq j$. This simply follows from the definition of $\mathcal{T}_{\boldsymbol{k}, i}$ in (75) that for any $(\boldsymbol{b}, B) \in \mathcal{T}_{\boldsymbol{k}, i}$ and $\left(\boldsymbol{b}^{\prime}, B^{\prime}\right) \in \mathcal{T}_{\boldsymbol{k}, j}$, say $B=\left\{\left(i_{t}, j_{t}\right): t=1, \ldots, m\right\}$ and $B^{\prime}=\left\{\left(i_{t}, j_{t}\right): t=1, \ldots, m^{\prime}\right\}$, where the pairs $\left(i_{t}, j_{t}\right)$ are arranged in ascending order, we have that $j_{m}-j_{m-1}=i$ for $B$ and $j_{m^{\prime}}-j_{m^{\prime}-1}=j$ for $B^{\prime}$. This proves our claim. Finally, using (76) and the fact that the sets $\left\{\mathcal{T}_{\boldsymbol{k}, i}\right\}$ are disjoint, we obtain

$$
\begin{aligned}
T(z) & =\pi(\boldsymbol{k},\{(1, L)\})+\sum_{i=1}^{L-1} r_{\boldsymbol{k}}(i) \sum_{(\boldsymbol{b}, B) \in \mathcal{T}_{\boldsymbol{k}}, i} \pi(\boldsymbol{b}, B) \\
& =z^{\ell(\boldsymbol{k})}(-1)+\sum_{i=1}^{L-1} r_{\boldsymbol{k}}(i) \sum_{(\boldsymbol{b}, B) \in \mathcal{T}_{\boldsymbol{k}}} z^{\ell(\boldsymbol{b})+i}(-1)^{|B|+1} \\
& =-z^{L}-\sum_{i=1}^{L-1} r_{\boldsymbol{k}}(i) z^{i} T(z) .
\end{aligned}
$$

Hence

$$
T(z)=-\frac{z^{L}}{1+\sum_{i=1}^{L-1} r_{\boldsymbol{k}}(i) z^{i}} .
$$

Substituting the above into (73) proves (11) of Theorem 1.

\section{APPENDIX B \\ DEGREE OF $\operatorname{det}\left(\mathrm{I}-\mathrm{A}_{\boldsymbol{k}} z\right)$}

In this section, we will determine the degree of polynomial $\operatorname{det}\left(\mathrm{I}-\mathrm{A}_{\boldsymbol{k}} z\right)$ that is required in the proof of Theorem 1.

Proposition 10: Let $\mathrm{A}_{k}$ be the adjacency matrix for the digraph $G_{\boldsymbol{k}}$ associated with UW $\boldsymbol{k}$ defined in Section III. Then

$$
\operatorname{deg} \operatorname{det}\left(\mathrm{I}-\mathrm{A}_{\boldsymbol{k}} z\right)=L \text {. }
$$

Proof: First, from (9) and (11), the two equivalent formulas for the enumeration of $s_{\boldsymbol{k}, n}$, we see $\operatorname{det}\left(\mathrm{I}-\mathrm{A}_{\boldsymbol{k}} z\right)$ is divisible by $h_{\boldsymbol{k}}(z)=(1-2 z)\left(1+\sum_{i=1}^{L-1} r_{\boldsymbol{k}}(i) z^{i}\right)+z^{L}$. It follows that

$$
\operatorname{deg} \operatorname{det}\left(\mathrm{I}-\mathrm{A}_{\boldsymbol{k}} z\right) \geq L
$$

To establish the converse of the above inequality, i.e., $\operatorname{deg} \operatorname{det}\left(\mathrm{I}-\mathrm{A}_{\boldsymbol{k}} z\right) \leq L$, it suffices to show that $\operatorname{rank}\left(\mathrm{A}_{\boldsymbol{k}}^{L-1}\right) \leq$ $L$, which in turns implies $\operatorname{rank}\left(\mathrm{A}_{\boldsymbol{k}}^{L}\right) \leq L$. As a result, the algebraic multiplicity of eigenvalue 0 for $A_{k}$ is at least $2^{L-1}-L$. Hence, the degree of $\operatorname{det}\left(\mathrm{I}-\mathrm{A}_{\boldsymbol{k}} z\right)$ is at most $L$.

To prove the claim, given the UW $\boldsymbol{k}=k_{1} \ldots k_{L}$ of length $L$ and the corresponding adjacency matrix $A_{k}$ for digraph $G_{k}$, let

$$
\mathrm{H}=\mathrm{A}_{\boldsymbol{k}}+\underline{e}_{\boldsymbol{k}_{1}} \underline{e}_{\boldsymbol{k}_{2}}^{\top}
$$

where $\boldsymbol{k}_{1}=k_{1}^{L-1}$ and $\boldsymbol{k}_{2}=k_{2}^{L}$, and where by $\underline{e}_{\boldsymbol{d}} \in \mathbb{F}^{2^{L-1}}$ with $\boldsymbol{d}=d_{1} \ldots d_{L-1} \in \mathbb{F}^{L-1}$ we mean $\left(\underline{e}_{\boldsymbol{d}}\right)_{j+1}=1$ if $j$ has the binary representation $\boldsymbol{d}$, and $\left(\underline{e}_{\boldsymbol{d}}\right)_{j+1}=0$, otherwise.

Apparently, $\mathrm{H}$ is the adjacency matrix for the digraph without UW forbidden constraint and is therefore independent of the choice of $\boldsymbol{k}$. As an example, if $L=3$, then

$$
\mathrm{H}=\left[\begin{array}{llll}
1 & 1 & 0 & 0 \\
0 & 0 & 1 & 1 \\
1 & 1 & 0 & 0 \\
0 & 0 & 1 & 1
\end{array}\right]
$$


Furthermore, it can be easily verified that $\mathrm{H}^{L-1}=\underline{\mathbf{1}} \underline{\mathbf{1}}^{\top}$ is the all-one matrix. Armed with the above, we now have

$$
\begin{aligned}
\mathrm{A}_{\boldsymbol{k}}^{L-1} & =\left(\mathrm{H}-\underline{e}_{\boldsymbol{k}_{1}} \underline{e}_{\boldsymbol{k}_{2}}^{\top}\right)^{L-1} \\
& =\mathrm{H}^{L-1}-\sum_{i=0}^{L-2} \mathrm{H}^{L-2-i}\left(\underline{e}_{\boldsymbol{k}_{1}} \underline{\boldsymbol{e}}_{\boldsymbol{k}_{2}}^{\top}\right) \mathrm{A}_{\boldsymbol{k}}^{i},
\end{aligned}
$$

where the last equality is due to the following identity for square matrices $A$ and $B$ :

$$
(A-B)^{L-1}=A^{L-1}-\sum_{i=0}^{L-2} A^{L-2-i} B(A-B)^{i} .
$$

Applying the standard rank inequality of $\operatorname{rank}(A+B) \leq$ $\operatorname{rank}(A)+\operatorname{rank}(B)$ [19] to (78) yields

$$
\begin{aligned}
& \operatorname{rank}\left(\mathrm{A}_{\boldsymbol{k}}^{L-1}\right) \\
& \quad \leq \operatorname{rank}\left(\mathrm{H}^{L-1}\right)+\sum_{i=0}^{L-2} \operatorname{rank}\left(\mathrm{H}^{L-2-i}\left(\underline{e}_{\boldsymbol{k}_{1}} \underline{\boldsymbol{e}}_{\boldsymbol{k}_{2}}^{\top}\right) \mathrm{A}_{\boldsymbol{k}}^{i}\right) \\
& \quad=1+\sum_{i=0}^{L-2} 1=L,
\end{aligned}
$$

and the proof is completed.

\section{APPENDIX C \\ VERIFICATION OF Algorithms 5 AND 6}

For completeness, we verify Algorithms 5 and 6 in this section.

For message $u_{1}$, i.e., the most likely message, we have from line 1 in Algorithm 5 that $m=1$ and $n=0$ since $F_{\boldsymbol{k}, 0}=c_{\boldsymbol{k}, 0}=1$. This results in the encoding output of the null codeword. In parallel, when receiving the null codeword, we have $n=0$. Algorithm 6 then sets $m=1$ at line 2 as $F_{\boldsymbol{k},-1}=0$. This verifies the correctness of Algorithms 5 and 6 for message $u_{1}$.

For $m \geq 2$, we shall show that for each $n \geq 1$, the encoding function $\phi_{\boldsymbol{k}}$ is a bijection between $\mathcal{U}_{\boldsymbol{k}}(n)=\left\{u_{m}: F_{\boldsymbol{k}, n-1}<\right.$ $\left.m \leq F_{\boldsymbol{k}, n}\right\}$ and $\mathcal{C}_{\boldsymbol{k}}(n)$, and the decoding function $\psi_{\boldsymbol{k}}$ is the functional inverse of $\phi_{\boldsymbol{k}}$. Equivalently, it suffices to show that

1) $\psi_{\boldsymbol{k}}$ is a bijection between $\mathcal{C}_{\boldsymbol{k}}(n)$ and $\mathcal{U}_{\boldsymbol{k}}(n)$ for each $n \geq 1$, and

2) $\phi_{\boldsymbol{k}}$ is the functional inverse of $\psi_{\boldsymbol{k}}$

We will proceed with this approach.

Prior to establishing the claims, we first introduce below a well-ordering of binary sequences. This is in fact a key concept embedded in Algorithms 5 and 6.

Definition 8 (Lexicographical ordering): For any two binary sequences $\boldsymbol{a}=a_{1} \ldots a_{i}$ and $\boldsymbol{b}=b_{1} \ldots b_{j}$, we say $\boldsymbol{a} \succ \boldsymbol{b}$ if $i>j$, or if $i=j$ and there exists a smallest integer $s$, $1 \leq s \leq i$, such that $a_{u}=b_{u}$ for $u=1, \ldots, s-1, a_{s}=1$, and $b_{s}=0$.

Obviously, such ordering is a total-ordering of binary sequences. How the lexicographical ordering of binary sequences plays a key role in the encoding and decoding of UDOOCs is due to the following lemma.
Lemma 1: For any two length- $n$ codewords $\boldsymbol{a}, \boldsymbol{b} \in \mathcal{C}_{\boldsymbol{k}}(n)$, we have $\boldsymbol{a} \succ \boldsymbol{b}$ if, and only if,

$$
\sum_{i=1}^{n} a_{i}\left|\mathcal{C}_{\boldsymbol{k}}\left(a_{1}^{i-1} 0, n\right)\right|>\sum_{i=1}^{n} b_{i}\left|\mathcal{C}_{\boldsymbol{k}}\left(b_{1}^{i-1} 0, n\right)\right| .
$$

Proof: As $\ell(\boldsymbol{a})=\ell(\boldsymbol{b})$ and $\boldsymbol{a} \succ \boldsymbol{b}$, there exists a smallest integer $s, 1 \leq s \leq n$, such that $a_{u}=b_{u}$ for $u=1, \ldots, s-1$, $a_{s}=1$, and $b_{s}=0$. Thus,

$$
\begin{aligned}
& \sum_{i=1}^{n} a_{i}\left|\mathcal{C}_{\boldsymbol{k}}\left(a_{1}^{i-1} 0, n\right)\right| \\
& \quad \geq \sum_{i=1}^{s-1} a_{i}\left|\mathcal{C}_{\boldsymbol{k}}\left(a_{1}^{i-1} 0, n\right)\right|+\left|\mathcal{C}_{\boldsymbol{k}}\left(a_{1}^{s-1} 0, n\right)\right| \\
& \quad>\sum_{i=1}^{s-1} a_{i}\left|\mathcal{C}_{\boldsymbol{k}}\left(a_{1}^{i-1} 0, n\right)\right|+\sum_{i=s+1}^{n} b_{i}\left|\mathcal{C}_{\boldsymbol{k}}\left(a_{1}^{s-1} 0 b_{s+1}^{i-1} 0, n\right)\right| \\
& \quad=\sum_{i=1}^{n} b_{i}\left|\mathcal{C}_{\boldsymbol{k}}\left(b_{1}^{i-1} 0, n\right)\right|
\end{aligned}
$$

where the second inequality follows from the fact that the sets $\mathcal{C}_{\boldsymbol{k}}\left(a_{1}^{s-1} 0 b_{s+1}^{i-1} 0, n\right)$, where $i=s+1, \ldots, n$ and $b_{i}=1$, are disjoint proper subsets of $\mathcal{C}_{\boldsymbol{k}}\left(a_{1}^{s-1} 0, n\right)$.

With the above lemma, given a codeword $c=c_{1} \ldots c_{n}$, Algorithm 6 outputs $\psi_{\boldsymbol{k}}(\boldsymbol{c})=m$ with

$$
\begin{aligned}
m & =\sum_{i=1}^{n} c_{i} \underline{x}_{\boldsymbol{k}}^{\top}\left(\prod_{j=1}^{i-1} \mathrm{~A}_{\boldsymbol{k}, c_{j}}\right) \mathrm{A}_{\boldsymbol{k}, 0} \mathrm{~A}_{\boldsymbol{k}}^{(n+L-1)-i} \underline{y}_{\boldsymbol{k}}+F_{\boldsymbol{k}, n-1}+1 \\
& =\sum_{i=1}^{n} c_{i}\left|\mathcal{C}_{\boldsymbol{k}}\left(c_{1}^{i-1} 0, n\right)\right|+F_{\boldsymbol{k}, n-1}+1 .
\end{aligned}
$$

We remark that the first term in the above, i.e., $\sum_{i=1}^{n} c_{i}\left|\mathcal{C}_{\boldsymbol{k}}\left(c_{1}^{i-1} 0, n\right)\right|$, is the only term dependent on $\boldsymbol{c}$, and it also appears in (79). It means that the encoding and decoding algorithms of UDOOC given in Algorithms 5 and 6 are indeed based on the lexicographical ordering of length- $n$ codewords in $\mathcal{C}_{\boldsymbol{k}}(n)$. Using Lemma 1 we can establish the range of $\psi_{\boldsymbol{k}}$ when restricted to $\mathcal{C}_{\boldsymbol{k}}(n)$.

Corollary 4: The range of $\psi_{\boldsymbol{k}}$ when restricted to $\mathcal{C}_{\boldsymbol{k}}(n)$ is the set $\mathcal{U}_{\boldsymbol{k}}(n)=\left\{u_{m}: F_{\boldsymbol{k}, n-1}<m \leq F_{\boldsymbol{k}, n}\right\}$. Therefore, $\psi_{\boldsymbol{k}}$ is a bijection between $\mathcal{C}_{\boldsymbol{k}}(n)$ and $\mathcal{U}_{\boldsymbol{k}}(n)$ for all $n \geq 1$.

Proof: Given $\mathcal{C}_{\boldsymbol{k}}(n)$, let $\boldsymbol{b}$ be the smallest member and $\boldsymbol{d}$ be the largest member according to the lexicographical ordering, i.e. $\boldsymbol{b} \preceq \boldsymbol{c} \preceq \boldsymbol{d}$ for all $\boldsymbol{c} \in \mathcal{C}_{\boldsymbol{k}}(n)$. It then follows from Lemma 1 that

$$
\min _{\boldsymbol{c} \in \mathcal{C}_{\boldsymbol{k}}(n)} \psi_{\boldsymbol{k}}(\boldsymbol{c})=\psi_{\boldsymbol{k}}(\boldsymbol{b}) \quad \text { and } \quad \max _{\boldsymbol{c} \in \mathcal{C}_{\boldsymbol{k}}(n)} \psi_{\boldsymbol{k}}(\boldsymbol{c})=\psi_{\boldsymbol{k}}(\boldsymbol{d}) \text {. }
$$

For the minimum, from (80) we have

$$
\psi_{\boldsymbol{k}}(\boldsymbol{b})=\sum_{i=1}^{n} b_{i}\left|\mathcal{C}_{\boldsymbol{k}}\left(b_{1}^{i-1} 0, n\right)\right|+F_{\boldsymbol{k}, n-1}+1 .
$$

Since $\boldsymbol{b}$ is the smallest member, it follows that for all $i, i=$ $1, \ldots, n,\left|\mathcal{C}_{\boldsymbol{k}}\left(b_{1}^{i-1} 0, n\right)\right|=0$ if $b_{i}=1$. Hence

$$
\min _{\boldsymbol{c} \in \mathcal{C}_{\boldsymbol{k}}(n)} \psi_{\boldsymbol{k}}(\boldsymbol{c})=\psi_{\boldsymbol{k}}(\boldsymbol{b})=F_{\boldsymbol{k}, n-1}+1 .
$$


To see the maximum, again from (80)

$$
\psi_{\boldsymbol{k}}(\boldsymbol{d})=\sum_{i=1}^{n} d_{i}\left|\mathcal{C}_{\boldsymbol{k}}\left(d_{1}^{i-1} 0, n\right)\right|+F_{\boldsymbol{k}, n-1}+1 .
$$

Since $\boldsymbol{d}$ is the largest member in $\mathcal{C}_{\boldsymbol{k}}(n)$, the sets $\mathcal{C}_{\boldsymbol{k}}\left(d_{1}^{i-1} 0, n\right)$, where $i=1, \ldots, n$ and $d_{i}=1$, are disjoint and proper subsets of $\mathcal{C}_{\boldsymbol{k}}(n)$. Moreover, for any $\boldsymbol{c} \in \mathcal{C}_{\boldsymbol{k}}(n)$ and $\boldsymbol{c} \prec \boldsymbol{d}$, there exists a smallest integer $s, 1 \leq s \leq n$, such that $d_{u}=c_{u}$ for $u=1, \ldots, s-1, d_{s}=1$, and $c_{s}=0$. This in turn implies $c \in \mathcal{C}_{\boldsymbol{k}}\left(d_{1}^{s-1} 0, n\right)$. Therefore,

$$
\bigcup_{\substack{i=1 \\ d_{i}=1}}^{n} \mathcal{C}_{\boldsymbol{k}}\left(d_{1}^{i-1} 0, n\right)=\mathcal{C}_{\boldsymbol{k}}(n) \backslash\{\boldsymbol{d}\}
$$

and

$$
\psi_{\boldsymbol{k}}(\boldsymbol{d})=c_{\boldsymbol{k}, n}-1+F_{\boldsymbol{k}, n-1}+1=F_{\boldsymbol{k}, n} .
$$

Finally, noting that $\left|\mathcal{C}_{\boldsymbol{k}}(n)\right|=\left|\mathcal{U}_{\boldsymbol{k}}(n)\right|$ and that $\psi_{\boldsymbol{k}}$ is injective by Lemma 1 , we conclude that $\psi_{\boldsymbol{k}}$ is bijective.

So far we have established the first claim that $\psi_{\boldsymbol{k}}$ is a bijection between $\mathcal{C}_{\boldsymbol{k}}(n)$ and $\mathcal{U}_{\boldsymbol{k}}(n)$. To prove the second claim that $\phi_{\boldsymbol{k}}$ is the functional inverse of $\psi_{\boldsymbol{k}}$, given a codeword $c=c_{1} \ldots c_{n}$, Algorithm 6 outputs

$$
m=\psi_{\boldsymbol{k}}(\boldsymbol{c})=\sum_{i=1}^{n} c_{i}\left|\mathcal{C}_{\boldsymbol{k}}\left(c_{1}^{i-1} 0, n\right)\right|+F_{\boldsymbol{k}, n-1}+1
$$

and $F_{\boldsymbol{k}, n-1}<m \leq F_{\boldsymbol{k}, n}$. Line 2 of Algorithm 5 would produce the correct $n$ for $m$. Then, from line 3 of Algorithm 5 , we get

$$
\rho_{0}=\sum_{i=1}^{n} c_{i}\left|\mathcal{C}_{\boldsymbol{k}}\left(c_{1}^{i-1} 0, n\right)\right|+1 .
$$

For the loop of lines 3-10 of Algorithm 5, when $i=1$, dummy has value

$$
\text { dummy }=\underline{x}_{\boldsymbol{k}}^{\top} \mathrm{A}_{\boldsymbol{k}, 0} \mathrm{~A}_{\boldsymbol{k}}^{n+L-2} \underline{y}_{\boldsymbol{k}}=\left|\mathcal{C}_{\boldsymbol{k}}(0, n)\right| .
$$

We distinguish two cases:

1) if $c_{1}=0$, then we must have

$$
\rho_{0}=\sum_{i=2}^{n} c_{i}\left|\mathcal{C}_{\boldsymbol{k}}\left(0 c_{2}^{i-1} 0, n\right)\right|+1 \leq \text { dummy }
$$

since $\sum_{i=2}^{n} c_{i}\left|\mathcal{C}_{\boldsymbol{k}}\left(0 c_{2}^{i-1} 0, n\right)\right|$ is the sum of the cardinalities of certain disjoint subsets (with different prefixes) of $\mathcal{C}_{\boldsymbol{k}}(0, n)$. Hence lines 5-9 of Algorithm 5 output $c_{1}=0$ as desired.

2) if $c_{1}=1$, then

$$
\rho_{0}=\left|\mathcal{C}_{\boldsymbol{k}}(0, n)\right|+\sum_{i=2}^{n} c_{i}\left|\mathcal{C}_{\boldsymbol{k}}\left(1 c_{2}^{i-1} 0, n\right)\right|+1>\text { dummy }
$$

and lines 5-9 of Algorithm 5 gives the correct $c_{1}=1$.

Furthermore, it can be seen that at the end of line 9, we have

$$
\rho_{1}=\sum_{i=2}^{n} c_{i}\left|\mathcal{C}_{\boldsymbol{k}}\left(c_{1}^{i-1} 0, n\right)\right|+1
$$

for the next iteration. Now suppose we are at the $t$ th iteration of Algorithm 5 for some integer $t$ with $1<t<n$. We have already determined $c_{1}, c_{2}, \ldots, c_{t-1}$, and have

$$
\rho_{t-1}=\sum_{i=t}^{n} c_{i}\left|\mathcal{C}_{\boldsymbol{k}}\left(c_{1}^{i-1} 0, n\right)\right|+1 .
$$

Line 4 of Algorithm 5 then gives

$$
\begin{aligned}
\text { dummy } & =\underline{x}_{\boldsymbol{k}}^{\top}\left(\prod_{i=1}^{t-1} \mathrm{~A}_{\boldsymbol{k}, c_{i}}\right) \mathrm{A}_{\boldsymbol{k}, 0} \mathrm{~A}_{\boldsymbol{k}}^{(n+L-1)-t} \underline{y}_{\boldsymbol{k}} \\
& =\left|\mathcal{C}_{\boldsymbol{k}}\left(c_{1}^{t-1} 0, n\right)\right| .
\end{aligned}
$$

Using the same reasoning as the above it can be easily shown that lines 5-9 of Algorithm 5 always produce the correct value for $c_{t}$. Finally at the $n$th iteration we have

$$
\rho_{n-1}=c_{n}\left|\mathcal{C}_{\boldsymbol{k}}\left(c_{1}^{n-1} 0, n\right)\right|+1
$$

and

$$
\text { dummy }=\underline{x}_{\boldsymbol{k}}^{\top}\left(\prod_{i=1}^{n-1} \mathrm{~A}_{\boldsymbol{k}, c_{i}}\right) \mathrm{A}_{\boldsymbol{k}, 0} \mathrm{~A}_{\boldsymbol{k}}^{L-1} \underline{y}_{\boldsymbol{k}}=\left|\mathcal{C}_{\boldsymbol{k}}\left(c_{1}^{n-1} 0, n\right)\right| .
$$

It should be noted that $c_{1}^{n-1} 0$ is a length- $n$ word, hence dummy $=0$ or 1 . We distinguish the following cases:

1) If dummy $=0$, then $c_{1}^{n-1} 0$ cannot be a valid codeword for UDOOC. Lines 5-9 of Algorithm 5 achieve exactly the above, since we have

$$
\rho_{n-1}=c_{n} \cdot \text { dummy }+1=1>\text { dummy }=0
$$

and the algorithm always outputs $c_{n}=1$.

2) If dummy $=1$, then $\rho_{n-1}=c_{n}+1$. The same reasoning as the above shows that lines 5-9 of Algorithm 5 always produce the correct value for $c_{n}$.

We therefore complete the proof that $\phi_{k}$ is the functional inverse of $\psi_{\boldsymbol{k}}$.

\section{APPENDIX D $\lim _{t \rightarrow \infty} L_{\boldsymbol{k}, t}$ FOR ALL-ZERO UW AND UNIFORM I.I.D. SOURCE}

Let $\boldsymbol{a}=00 \ldots 0$ be the all-zero UW of length $L$. From (23), (54), and (55), it can be easily verified that

$$
\sum_{n=0}^{\infty} c_{\boldsymbol{a}, n} z^{n}=1+\frac{z}{1-\sum_{i=1}^{L} z^{i}}
$$

Furthermore, from (23) we have $g(z)=(1-z) h_{\boldsymbol{a}}(z)=$ $1-2 z+z^{L+1}$. It is straightforward to show that the two polynomials $g(z)$ and $\frac{d}{d z} g(z)$ are co-prime to each other; hence there are no repeating zeros in $h_{\boldsymbol{a}}(z)$. It then implies that all the nonzero eigenvalues of $\mathrm{A}_{\boldsymbol{a}}$ are simple.

Denote by $\lambda_{1} \cdots \lambda_{L}$ the nonzero eigenvalues of $A_{\boldsymbol{a}}$, and assume without loss of generality that $\left|\lambda_{1}\right|>\left|\lambda_{2}\right| \geq \cdots \geq$ $\left|\lambda_{L}\right|$. Then

$$
c_{\boldsymbol{a} . n}=\delta_{n}+\sum_{i=1}^{L} a_{i}\left(\lambda_{i}\right)^{n}
$$


where $a_{1} \cdots a_{L}$ are constants such that (81) holds. We can also obtain the closed-form expression for $F_{\boldsymbol{a}, n}$ as

$$
F_{\boldsymbol{a}, n}=1+\sum_{i=1}^{L} a_{i} \frac{\lambda_{i}^{n+1}-1}{\lambda_{i}-1}, \quad \text { for all } n \geq 0
$$

Consider a uniform i.i.d. source $\mathcal{U}$ of alphabet size $M$ with $M>1$. Let $\mathcal{U}^{t}$ be the grouped source obtained by grouping any $t$ source symbols (with repetition) in $\mathcal{U}$. It is clear that $\mathcal{U}^{t}$ is also a uniform i.i.d. source. The per-letter average codeword length is given by

$$
\begin{aligned}
L_{\boldsymbol{a}, t} & =\frac{1}{t}\left(L+\sum_{i=2}^{M^{t}} p_{i} \ell\left(\phi\left(u_{i}\right)\right)\right) \\
& =\frac{1}{t}\left(L+\frac{1}{M^{t}} \sum_{i=2}^{M^{t}} \ell\left(\phi\left(u_{i}\right)\right)\right) .
\end{aligned}
$$

Let $N$ be the smallest integer such that $F_{\boldsymbol{a}, N} \geq M^{t}>$ $F_{\boldsymbol{a}, N-1}$. Then

$$
\begin{aligned}
L_{\boldsymbol{a}, t} & \geq \frac{1}{t}\left(L+\frac{1}{F_{\boldsymbol{a}, N}} \sum_{i=2}^{M^{t}} \ell\left(\phi\left(u_{i}\right)\right)\right) \\
& \geq \frac{1}{t}\left(L+\frac{1}{F_{\boldsymbol{a}, N}} \sum_{i=2}^{N-1} i \cdot c_{\boldsymbol{a}, i}\right) \\
& \geq \frac{1}{\log _{M}\left(F_{\boldsymbol{a}, N}\right)}\left(L+\frac{1}{F_{\boldsymbol{a}, N}} \sum_{i=2}^{N-1} i \cdot c_{\boldsymbol{a}, i}\right) .
\end{aligned}
$$

Consequently,

$$
\begin{aligned}
& \lim _{t \rightarrow \infty} L_{\boldsymbol{a}, t} \\
& \geq \lim _{N \rightarrow \infty} \frac{1}{\log _{M}\left(F_{\boldsymbol{a}, N}\right)}\left(L+\frac{1}{F_{\boldsymbol{a}, N}} \sum_{i=2}^{N-1} i \cdot c_{\boldsymbol{a}, i}\right) \\
& =\lim _{N \rightarrow \infty} \frac{\sum_{i=2}^{N-1} i \cdot c_{\boldsymbol{a}, i}}{F_{\boldsymbol{a}, N} \log _{M}\left(F_{\boldsymbol{a}, n}\right)} \\
& =\lim _{N \rightarrow \infty} \frac{\sum_{i=1}^{L} a_{i} \frac{\lambda_{i}\left[(N-1) \lambda_{i}^{N}-N \lambda_{i}^{N-1}+1\right]}{\left(\lambda_{i}-1\right)^{2}}}{\left(1+\sum_{i=1}^{L} a_{i} \frac{\lambda_{i}^{N+1}-1}{\lambda_{i}-1}\right) \log _{M}\left(1+\sum_{i=1}^{L} a_{i} \frac{\lambda_{i}^{N+1}-1}{\lambda_{i}-1}\right)} \\
& =\frac{1}{\log _{M}\left(\lambda_{1}\right)}=\frac{1}{\log _{M}\left(g_{\boldsymbol{a}}\right)} .
\end{aligned}
$$

This implies

$$
\lim _{t \rightarrow \infty} L_{\boldsymbol{a}, t} \geq \frac{\log _{2}(M)}{\log _{2}\left(g_{\boldsymbol{a}}\right)}=\frac{\mathrm{H}(\mathcal{U})}{\log _{2}\left(g_{\boldsymbol{a}}\right)}
$$

\section{REFERENCES}

[1] N. Alon and A. Orlitsky, "A lower bound on the expected length of oneto-one codes," IEEE Trans. Inf. Theory, vol. 40, no. 5, pp. 1670-1672, September 1994.

[2] J. Bang-Jensen and G. Z. Gutin, Theory, Algorithms and Applications, Springer Monographs in Mathematics, 2009.

[3] Information Technology-Telecommunications And Information Exchange Between Systems-Local and Metropolitan Area Networks-Specific Requirements-Part 11: Wireless LAN Medium Access Control (MAC) and Physical Layer (PHY) Specifications, IEEE Standard 802.11-1999.
[4] N. Biggs, Algebraic Graph Theory, Cambridge Mathematical Library, 1994.

[5] C. Blundo and R. D. Prisco, "New bounds on the expected length of one-to-one codes," IEEE Trans. Inf. Theory, vol. 42, no. 1, pp. 246-250, January 1996.

[6] J. Cheng, T.-K. Huang and C. Weidmann, "New bounds on the expected length of optimal one-to-one codes," IEEE Trans. Inf. Theory, vol. 53, no. 5, pp. 1884-1895, May 2007.

[7] T. M. Cover and J. A. Thomas, Elements of Information Theory, New York, NY: John Wiley \& Sons, 1991.

[8] R. Doroslovački, "The set of all the words of length $n$ over any alphabet with a forbidden good subword," Univ. u Novom Sadu, Zb. Rad. Prirod.Mat. Fak. Ser. Mat., 23:2, pp. 239-244, 1993.

[9] R. Doroslovački, "The set of all the words of length $n$ over alphabet $\{0,1\}$ with any forbidden subword of length three," Univ. u Novom Sadu, Zb. Rad. Prirod.-Mat. Fak. Ser. Mat., 25: 2, pp. 111-115, 1995.

[10] R. Doroslovački, "Binary $n$-Words without the subword $1010 \cdots 10$," Novi Sad J. Math., vol. 28, no. 2, pp. 127-133, 1998.

[11] R. Doroslovački, "On binary $n$-words with forbidden 4-subwords," Novi Sad J. Math., vol. 29, no. 1, pp. 27-32, 1999.

[12] R. Doroslovački, " $n$-words over any alphabet with forbidden any 3subwords," Novi Sad J. Math., vol. 30, no. 2, pp. 159-163, 2000

[13] J. G. Dunham, "Optimal noiseless coding of random variables," IEEE Trans. Inf. Theory, vol. IT-26, no. 3, p. 345, May 1980.

[14] Sam E. Ganis, Notes on the Fibonacci Sequence, Amer. Math. Monthly, 1959, pp. 129-130.

[15] C. Godsil and G. F. Royle, Algebraic Graph Theory, Springer, 2001

[16] I. Goulden and D. M. Jackson, "An inversion theorem for cluster decompositions of sequences with distinguished subsequences," J. London Math. Soc, pp. 567-576, 1979.

[17] L. J. Guibas and A. M. Odlyzko, "Periods in strings," J. Combinatorial Theory, series A 30, pp. 19-42, 1981.

[18] K. M. Hoffman and R. Kunze, Linear Algebra, 2nd edition, Pearson, 1971.

[19] R. A. Horn and C. R. Johnson, Matrix Analysis, 2nd edition, Cambridge University Press, 2012.

[20] E. J. Kupin and D. S. Yuster, "Generalizations of the Goulden-Jackson cluster method," J. Difference Eq. Appl., 16:12, pp. 1463-1480, 2010.

[21] S. K. Leung-Yan-Cheong and T. M. Cover, "Some equivalences between Shannon entropy and Kolmogorov complexity," IEEE Trans. on Information theory, vol. IT-24, no. 3, pp. 331-338, May 1978.

[22] J. Noonan, "New upper bounds for the connective constants of selfavoiding walks," J. Statistical Physics, vol. 91, nos. 5/6, 1998.

[23] J. Noonan and D. Zeilberger, "The Goulden-Jackson cluster method: extensions, applications, and implementations," J. Difference Eq. Appl., 5: pp. 355-377, 1999.

[24] J. Rissanen, "Tight lower bounds for optimum code length," IEEE Trans. Inf. Theory, vol. IT-28, no. 2, pp. 348-349, March 1982.

[25] E. Rivals and S. Rahmann, "Combinatorics of periods in strings," J. Combinatorial Theory, series A 104, pp. 95-113, 2003.

[26] S. A. Savari, "On one-to-one codes for memoryless cost channels," IEEE Trans. Inf. Theory, vol. 54, no. 1, pp. 367-379, January 2008.

[27] S. A. Savari and A.Naheta, "Bounds on the expected cost of one-toone codes," IEEE International Symposium on Information Theory, June 2004.

[28] R. Stanley, Enumerative Combinatorics, vols. 1 and 2, Cambridge Studies in Advanced Mathematics, 2011.

[29] W. Szpankowski, "A one-to-one code and its anti-redundancy," IEEE Trans. Inf. Theory, vol. 54, no. 10, pp. 4762-4766, October 2008.

[30] W. Szpankowskia and S. Verd u, "Minimum expected length of fixed-tovariable lossless compression of memoryless sources," IEEE International Symposium on Information Theory, Seoul, Korea, July 2009.

[31] E. I. Verriest, "An achievable bound for optimal noiseless coding of a random variable," IEEE Trans. Inf. Theory, vol. IT-32, no. 4, pp. 592-594, July 1986.

[32] X. Wen, "The symbolic Goulden-Jackson cluster method," J. Difference Eq. Appl., 11:2, pp. 173-179, 2006.

[33] A. D. Wyner, "An upper bound on the entropy series," Inf. Control, vol. 20, 30: pp. 176-181, 1972.

[34] http://bcl.comli.eu/download-en.html

[35] http://corpus.canterbury.ac.nz/descriptions/

[36] http://oxforddictionaries.com/words/what-is-the-frequency-of-theletters-of-the-alphabet-in-english 